\title{
Abstracts of the International Medical Students' Congress of Bucharest (IMSCB) 2019
}

01. A CASE OF HBV/HCV COINFECTION: AN UNEXPECTED AFTERMATH Julia Susanne A. Balanescu' ${ }^{1}$ and Lect. Ion Relu Ondin Zaharia ${ }^{2}$

"Carol Davila" University of Medicine and Pharmacy, Bucharest, Romania. 2Department of Internal Medicine, "Professor Dr. Theodor Burghele" Clinical Hospital, Bucharest, Romania.

BACKGROUND: Hepatitis B virus (HBV) along with hepatitis C virus (HCV) infections often lead to the development of chronic liver disease worldwide. $\mathrm{HBV} / \mathrm{HCV}$ co- infection occurs due to the common routes of transmission and this particular situation represents a great challenge for clinicians considering the complex interactions of HBV and HCV. The severity of liver disease and the increased rate of complications, compared to monoinfection, contributes to its complexity and to the challenge of its treatment. CASE PRESENTATION: A 69-year-old female patient was admitted to the internal medicine department to investigate the cause of progressive fatigue, associated with dizziness. Medical history comprised Hashimoto's thyroiditis treated with Euthyrox, insulin- dependent diabetes mellitus type II, essential hypertension under treatment and a post- transfusion acute hepatitis in 1980, following a complicated abortion. The patient didn't undergo any therapy for the post-transfusion viral infection and in 2017 she was diagnosed with chronic viral hepatitis with HBV and HCV, stating she had no symptoms. The physical exam of the patient revealed abdominal excoriations and signs of insulin-use, and no clinical manifestations of ascites, splenomegaly or hepatic encephalopathy could be observed. Blood tests showed unbalanced blood sugar levels with an $\mathrm{HbA} 1=9.3 \%$, thrombocytopenia, hepatic cytolysis, increased coagulation time, hypocholesterolemia, an elevated level of gamma-glutamyltransferase and the presence of HBsAg and anti-VHC antibody. Abdominal ultrasonography indicated a thin lining of perihepatic fluid and portal hypertension. A CT scan was performed and there were no signs of an advanced degree of necroinflammation, therefore the diagnose of a decompensated cirrhosis was unlikely. Differential diagnoses included the severe complication of the disease, hepatocellular carcinoma, as well as the possibly mixt aetiology of liver disease, other factors contributing to its progress. Treatment incorporated a more controlled administration of insulin shots regarding the correct monitoring of her metabolic disease. Doubtless, the most importan issue of the treatment was focused on her antiviral therapy. CONCLUSION: We present a case of HBV/HCV co-infection, which has the particularity to be in a state of compensated liver function and to be overlapped with Hashimoto's thyroiditis and insulin-dependent diabetes mellitus type II, conditions which can be manifestations of the infection.

Key words: HBV/HCV, coinfection, cirrhosis, associated conditions.

02.

\section{A CASE OF MARFAN SYNDROME IDENTIFIED UPON OPHTHALMOLOGICAL} EXAMINATION

Andreea Maria A.D. Neamtu', Ioana Maria C.V. Mureșan' and Simona Bucerzan MD, PhD

"Iuliu Hațieganu" University of Medicine and Pharmacy, Cluj-Napoca, Romania.

BACKGROUND: Marfan syndrome is a disorder that affects the connective tissue which provides strength and flexibility to bones, ligaments, muscles, blood vessels, and heart valves. It is an autosomal dominantly inherited connective tissue disorder with an incidence of approximately 1:5000. Around $25 \%$ of Marfan syndrome cases result from a new mutation in the FBN 1 gene. The features of this genetic disorder can start manifesting anytime between infancy and adulthood. CASE PRESENTATION: We describe a case of a 4 years old male patient who presented to the ophthalmology with vision problems. The diagnosis was bilateral subluxation of the lenses and the ophthalmologist referred him to the pediatric genetic department for further investigations. On clinical examination we identified joint hypermobility (loose joints), arachnodactyly, positive wrist (WalkerMurdoch) and thumb (Steinberg) signs, sunken chest (pectus excavatum). The phenotypic changes suggesting the disorder were validated by paraclinical investigation. Echocardiography showed dilatation of the aorta with a Z score of 4.25 , minor mitral valve prolapse, tricuspid valve regurgitation. We are waiting for the genetic test results to confirm the diagnosis. CONCLUSION: The particularity of this case is the fact that the suspicion of Marfan syndrome appeared after a control at the ophthalmology for vision problems, caused by dislocated lenses in both eyes.

Key words: Marfan syndrome, collagen disorder, bilateral dislocated lenses

\section{A HAPPY ENDING TO BOERHAAVE SYNDROME- CASE REPORT}

loana I. Chirvasa', Diana-Elena G.L. Spînu', Moufida B.D Benhenni, Nada A.N, Achouri' ${ }^{1}$, Mircea Florin Costache MD² $^{2}$, Lect. Nuțu Vlad², and Lect. Dan-Constantin Andronic ${ }^{2}$

"Gr. T. Popa" University of Medicine and Pharmacy, Iași, Romania. Universitary Emergency Hospital "St. Spiridon", Iași, Romania.

BACKGROUND: Boerhaave syndrome is a spontaneous perforation of the esophagus that occurs with a sudden increase in intraesophageal pressure. It is one of the rarest and lethal diseases of the gastrointestinal tract. One of the main complications would be chemical mediastinitis but this patient fully recovered after 3 months being treated. CASE PRESENTATION: We present a case of a 59-year-old woman who came for medical examination because of symptoms like: dyspnea, anterior chest pain, cough, dysphagia, fever and altered general condition. She was treated for pneumonia but none of the results were positive. After performing a chest $x$-ray a large left pleural effusion was discovered and a pleurotomy a minima with drain tube insertion was performed. The suspicion of a esophageal rupture was confirmed by a methylene blue administration and the patient was transferred immediately to the surgery clinic. She was tachycardic and the vesicular murmur of the left lung area was absent. Ten days after the first emetic episode, the upper gastrointestinal series showed a perforation in the lower $1 / 3$ rd of the esophagus and a left esopleural fistula with no signs of mediastinitis. After a hydro electrolytic rebalancing the following procedures were performed: cervical lateral esophagostomy, exploratory laparotomy with identification of the esophageal perforation and suture of the left transpleurally externalized Kehr tube, jejunal feeding tube, left thoracotomy with the functional releasing of the left lung and insertion of two drain tubes. The post-operative evolution was satisfying, the case being cured with the removal of the jejunostomy. CONCLUSION: Boerhaave syndrome is a very rare disease that needs to be treated immediately. Although the postemetic perforations have a higher mortality rate than the iatrogenic perforations, they can be cured if treated correctly, in this case by $\mathrm{T}$-tube placement.

Key words: Boerhaave syndrome, esopleural fistula, anterior chest pain, cough, vomiting, oesophageal rupture. 
04.

\section{CARCINOMA WITH BRCA1 MUTATION}

Marcu-Alexandru I.C. Posea', Posea Ioana Maria, Victor Vidican and Rares Buiga MD, PhD

'Iuliu Hațieganu" University of Medicine and Pharmacy, Cluj-Napoca, Romania.

BACKGROUND: High-grade serous carcinoma of ovarian type is a kind of tumor arising from ovaries, fallopian tube or peritoneum. It was previously thought to develop from the "coelomic epithelium" layering the ovary, but new findings indicate that its origins might be in the fallopian tube epithelium, at least in certain cases if not in all. Prophylactic salpingooophorectomy is associated with reduced risk of developing ovarian cancer in women with BRCA1 or BRCA2 mutations. Breast cancer type susceptibility protein is a protein that, in humans, is encoded by the BRCA gene, which is involved in DNA repairing and tumor suppression mechanisms. Mutations found in the BRCA 1 gene are associated with a high risk of developing breast and ovarian cancer. CASE PRESENTATION: Herein we present the case of a 50 -year-old woman with BRCA 1 class 5 mutation who underwent an unilateral breast sectorectomy with axillary lymphadenectomy after being diagnosed with breast cancer. Eleven years later, discovering that it carries a mutation of her BRCA1 gene, she had prophylactic bilateral mastectomy, followed by total hysterectomy and bilateral salpingo-oophorectomy. Pathological examination of the ovaries and fallopian tube showed no evidence of tumor or dysplasia. After anothe 10 months, not only did the patient develop a high-grade serous peritoneal carcinoma of ovarian type despite her prophylactic hysterectomy and salopingo-ooforectomy, but also underwent rapid tumor development. CONCLUSION: Although the patient followed the standard protocol, she stil developed an aggressive carcinoma. More research needs to be done in this field to determine the efficacy of standard prophylactic measures in patients with specific BRCA1 mutantions.

Key words: High-grade serous ovarian type carcinoma, peritoneal origin BRCA1 mutation, prophylactic hysterectomy and salpingo-oophorectomy.

\section{ABDOMINAL AND BACK PAIN AFTER ROUX EN Y GASTRIC-BY-PASS - DO} NOT FORGET INTERNAL HERNIA!

Rares Constantin R. Moldovanu', Stefana Eugenia R. Moldovanu', Iuliana M. Stefanuca', Ilinca L. Bắrbulescu', Daniel Timofte ${ }^{1,2}$

"Grigore T. Popa" University of Medicine and Pharmacy, Iași, Romania 2Department of General Surgery, "Sf. Spiridon" University Hospital, lasi, Romania.

BACKGROUND: Laparoscopic Roux-en-Y gastric bypass (LRYGB) is a common bariatric surgical procedures. LRYCB is associated with different complications: metabolic, nutritional and internal herniation. CASE PRESENTATION: A 48-year-old woman was admitted in emergency for abdominal and back pain. Physical examination showed no signs acute abdomen. Laboratory investigations revealed no abnormalities (CRP 2.0 $\mathrm{mg} / \mathrm{L}$, white blood count $4100 / \mathrm{mm} 3$ ). She's past medical history revealed: appendectomy at age of 16; laparoscopic adjustable gastric banding in 2005 (initial weight at $123 \mathrm{~kg} / \mathrm{BMl}$ at $48 \mathrm{~kg} / \mathrm{m} 2$ ); redo laparoscopic adjustable gastric banding in 2008 for lap band migration; cholecystectomy in 2009; sleeve gastrectomy in 2012 (weight at $106 \mathrm{~kg} / \mathrm{BMl}$ at $41.4 \mathrm{~kg} / \mathrm{m2}$ ); redo sleeve for gastric dilatation in 2016; LRYGB in 2018 for severe GERD after sleeve in 2018. Actually she's weight is stable at $66 \mathrm{~kg}$ (BMl at $25.8 \mathrm{~kg} / \mathrm{m2}$ ). A CT scan was performed and revealed dilated small bowel loops and the swirling of mesenteric vessels ("swirl sign"). An exploratory laparoscopy was performed and an internal herniation through Petersen's space was found. The closure of the mesenteric defects was performed by laparoscopic approach using a non resorbable barbed suture. The post- operative course was uneventful. CONCLUSION: Diagnosing an internal herniation is difficult due to the nonspecific clinical presentation. The CT scan can affirm the diagnostic. This sign is present in $74 \%$ of the cases with this herniation. A missed diagnosis of internal herniation may cause potentially serious complications. A patient with a gastric bypass who experiences intermittent abdominal complaints should undergo laparoscopy to rule out internal herniation.

Key words: Roux-en-Y gastric bypass, Peterson's space, Herniation, Laparoscopy.
06. ABDOMINAL BRUITS REVELING A HIDDEN SERIOUS COMPLICATION OF RHEUMATOID ARTHRITIS: THE IMPORTANCE OF COMPLETE PHYSICAL EXAMINATION

Bianca D. Mihart' ${ }^{1}$, Andrei C.I. Mitre'1, Matei R. Iurea', Laura Damian $\mathrm{MD}, \mathrm{PhD}^{2}$

'Iuliu Hatieganu" University of Medicine and Pharmacy, Cluj-Napoca, Romania. ${ }^{2}$ Emergency Clinical County Hospital, Cluj-Napoca, Romania.

BACKGOUND: Rheumatoid arthritis (RA) is an autoimmune disease involving the joints. Extra-articular manifestations develop in $40 \%$ of the patients resulting in increased morbidity and mortality. Rheumatoid vasculitis (RV) is a rare complication of RA, occurring in longstanding disease. Its incidence was reduced by the use of disease- modifying drugs. RV can involve any type of vessel, from small vessels (in cutaneous vasculitis and the bland vasculitis at the pressure sites) to medium-size vessels (similar to polyarteritis nodosa) and large vessels respectively (similar to giant cell arteritis). CASE PRESENTATION: A 63-year female patient from the rural area, diagnosed with RA since the age of 47 , with an inconstant disease-modifying drug therapy, presented for intense abdominal pain, mainly in the paraumbilical area, with lateral irradiation. Examination revealed joint deformities and limitation, with synovitis of the small hand joints, knees and ankles, but also intense bruits para-umbilical, in the flanks and ove the iliac arteries. Laboratory revealed intense inflammation (erythrocyte sedimentation rate $88 \mathrm{~mm} / \mathrm{h}$, C-reactive protein of $33 \mathrm{mg} / \mathrm{dL}$ (normal $<6$ $\mathrm{mg} / \mathrm{dl}$ ), elevated rheumatoid factor and anti-citrullinated peptide antibody titers and low $\mathrm{C}_{3}$ and $\mathrm{C}_{4}$ complement fractions. All the other tests (including anti-neutrophil cytoplasm antibodies ANCA, antinuclear antibodies, hepatitis $B$ and C tests, amylases, urinalysis) were normal. A medium-vesse RV was diagnosed on clinical basis and methylprednisolone and cyclophosphamide pulse-therapy was immediately introduced, with rapid resolution of the clinical picture. Notably, the abdominal bruits also disappeared. The abdominal echo-Doppler and enhanced angio-MR (performed from administrative reasons after 10 and 14 days) were normal as well. After completing the pulses she received azathioprine and hydroxychloroquine, and afterwards adalimumab and rituximab (anti-CD20) along with tapering glucocorticoids, with favorable outcome. CONCLUSION: RV involving abdominal vessels is a rare complication of RA with lethal potential. Even when ancillary examinations are normal, a simple clinical sign may help identify and treat early this rare disease.

Key words: rheumatoid vasculitis, abdominal bruits, clinical examination.

07. AN UNUSUAL CASE OF CHRONIC INFLAMMATORY DEMYELINATINC POLYNEUROPATHY ASSOCIATED WITH SERONEGATIVE CHURG-STRAUSS VASCULITIS

Andrei Mihai G.C. Borcan', Emma Marcelle J.D. Burke', Alexandra Oprisan ${ }^{1,2}$

"Carol Davila" University of Medicine and Pharmacy, Bucharest, Romania. ${ }^{2}$ Neurology Department, Colentina Clinical Hospital, Bucharest, Romania.

BACKGROUND: Chronic Inflammatory Demyelinating Polyneuropathy (CIDP) is a rare autoimmune neuropathy which causes the demyelination of the peripheral nerves and their roots, leading to important motor deficit characterized by a common fluctuating evolution, rarely a progressive one. Churg-Strauss systemic vasculitis (now known as eosinophilic granulomatosis with polyangiitis) is an extremely rare disorder that causes eosinophilic inflammation of the small vessels, resulting in axonal and internal organ ischemia. As each disease can have debilitating consequences on motor function, the synergistic action of both can worsen a patient's prognosis by the complexity of the pathologic processes and clinical presentation. CASE PRESENTATION: We present the case of a 52-yearold man with known chronic inflammatory demyelinating polyneuropathy, diagnosed in 2009 following a progressive and steady motor impairment mainly affecting the lower limbs and gait; Churg-Strauss vasculitis since 2009 , which posed as a diagnostic challenge because of the uncommon evolution, debuting with fever and digestive symptoms (in the absence of the characteristic respiratory features), for which repeated endoscopy examinations, imaging studies, and an enteral biopsy were performed; corticosteroid-induced osteoporosis, complicated by a pathologic tibial fracture in 2011 and corticosteroid- induced diabetes mellitus since 2015 which is believed to have further aggravated the patient's motor symptoms by its ischemic axonal neuropathic effect. After three years of no follow-up and no physical rehabilitation, the patient was admitted for aggravation of the known motor deficit. Physical examination revealed the following: normal cranial nerves function; $3 / 5$ lower limbs, 4/5 lower limbs flaccid 
tetraparesis (distalıproximal); deep tendon reflexes were present, yet diminished in the upper limb and abolished in the lower limbs; generalized atrophy (distalsproximal); superficial palmar and plantar hypoaesthesia and severe proprioceptive deficits; inability to stand or walk, even with bilateral aid.

CONCLUSION: The concomitant association of two autoimmune diseases which damage the peripheral nervous system by different pathogenic mechanisms (as inflammatory demyelinating in CIDP and axonal ischemia in Churg-Strauss vasculitis) is an extremely rare finding. As both the diagnostic methods and treatment options are completely different for each disease, this case represents a true challenge for the physician.

Key words: CIDP, neuropathy, Churg-Strauss vasculitis.

\section{AORTIC VALVULOPATHY IN OCHRONOSIS}

Mihaela M. Rotari', Ursu Sergiu Diana', Oleg Arnaut MD, PhD'

'"Nicolae Testemițanu“ State University of Medicine and Pharmacy, Chisinau, Moldova.

BACKGROUND: Ochronosis (alkaptonuria) is a very rare metabolic disease. This pathology has autosomal recessive transmission and is manifested by the progressive and irreversible impairment of the connective tissue. Alkaptonuria occurs as a result of the innate defect of tyrosine metabolism due to the homogentisic acid oxidase deficiency (enzymopathy), which causes the accumulation of homogentisic acid in the collagen structures of the body. Clinical manifestations of alkaptonuria are homogentisic aciduria, specific staining of conjunctival structures (ochronosis) and arthropathy of large joints. Cardiovascular disorders involving the aortic, mitral valve and coronary arteries in alkaptonuria are less common, and their incidence remains unclear. CASE PRESENTATION: In this clinical report we present the case of a 57-year-old female, with a history of progressive blue pigmentation of the integuments, sclera and auricular cartilages, as well as hyperchromic urine, which the patient reports having it since childhood. Four years ago, the patient had total bilateral hip arthroplasty, and at the moment presents with clinical signs of severe aortic stenosis and ischemic cardiomyopathy. CONCLUSION: We will refer to the etiology, pathogenesis, diagnosis and possibilities of treatment during ochronosis discussion. In particular, we will describe the cardiac conditions detected in this case, the intraoperative findings and the results of the surgical treatment performed.

Key words: alkaptonuria, autosomal recessive disease, blue pigmentation, aortic valvulopathy.

09. ARTERIAL DISEASE WITH MULTIPLE LOCATIONS AND THE HEART TEAM DECISIONS

Ana-Maria C. Neagu', Alexandra-Ionela T. Strugariu', Nicușor Lovin, MD', Razan Al Namat

'Grigore T. Popa" University of Medicine and Pharmacy, Iași, Romania.

BACKGROUND: Multiple location arterial disease is defined as the simultaneous presence of atherosclerotic lesions with clinical significance in at least two vascular major territories. The management and the treatment for such a patient needs to be strategical and must be chosen individually, based on both clinical and technical points of view. It is mandatory to have a multidisciplinary team to approach it. CASE PRESENTATION: We present the case of a 57 years old woman who was admitted to the Cardiology Clinic IBCV lasi for pain in the right lower limb when walking and in orthostatism started one month ago. The patient is known to have high blood pressure grade III with a very high additional risk, type II diabetes, metabolic syndrome. In addition, she has ischemic heart disease with tricoronary lesions, percutaneous angioplasty on the anterior descending and marginal oblique artery and angioplasty on the left carotid artery and also stenting of the left superficial femoral artery. After angiography of the lower limbs, significant stenoses were objectified at the level of the bifurcation of the right femoral artery with the indication of surgical treatment. CONCLUSION: In this patient with an arterial disease with a given location, the coexistence of an atherosclerotic determination in another vascular territory, has increased the risk of recurrence of symptoms and complications for the first manifest location of atherosclerotic disease. After all of the surgical interventions, she is still presenting with pain in the right lower limb, in addition to the comorbidities, clinical and biological signs she is presented with. After her hospitalization, her symptomatology improved due to the medication she was given. Given the risk factors and the overall clinical status of this patient, the decisions which concern the treatment should be discussed by a multidisciplinary team in order to develop the strategy perfectly elected for this individual case.

Key words: angioplasty, stenosis, stent, hypertension.

\section{ASSESING THE ETIOLOGY OF INTESTINAL INTRAMURAL HEMATOMAS IN A PSYCHIATRIC PATIENT}

Anca Florentina G. Botoi1, Irina V. Dicea', Grigore Botoi MD PhD

'"Carol Davila" University of Medicine and Pharmacy, Bucharest, Romania. 2Emergency County Hospital "Dr. Constantin Opriş".

BACKGROUND: While non-traumatic spontaneous intestinal hematomas usually reflect an underlying hematologic disease or over-anticoagulation, small-bowel intramural hematomas mostly result from blunt trauma. The aim of our case presentation is to raise awareness about their potentia complications and the challenges encountered in determining their etiology. CASE PRESENTATION: The 40 year-old patient presented to the Emergency Room for acute abdominal pain, along with vomiting, flatulence and no bowel transit for two weeks. The patient's medical history revealed no conditions, except significant congenital mental retardation. The patient had undergone several investigations, among which the abdominal ultrasound and CT scan showed a thickening of the transverse colon wall (up to 9mm) as well as gastric, duodenal and small-intestine occlusion, lacking any signs of pneumoperitoneum. The only noticeable change in lab results was an increased number of white blood cells (WBC) $14100 / \mathrm{mcL}$. The data suggested a high intestinal obstruction and after a 4 -hour intense hydroelectrolitical re-equilibration, exploratory laparotomy with mesenteric infiltration of procaine $1 \%$ and peritoneal lavage were performed. Multiple intramural hematomas were discovered in the middle portion of the jejuno-ileum. The postoperative evolution included restoration of bowel transit and ora alimentation. Six days later, the patient presented with acute surgical abdomen, clinical signs of peritoneal irritation and lack of bowel function. WBC escalated to $35800 / \mathrm{mcL}$, serum proteins decreased to $4.2 \mathrm{~g} / \mathrm{dL}$ and serum urea was $116 \mathrm{mg} / \mathrm{dl}$. Second exploratory laparotomy revealed abundant purulent and faecaloid fluid in the peritoneal cavity and multiple perforations of the hematomas discovered during the first surgery. Adhesiolysis, enterectomy, ileostomy and elastic abdominal belt contention were performed. Postoperative treatment included analgesics and antibiotics. The patient developed subsequent infections, which were treated according to the antibiogram. CONCLUSION: The patient's recovery was slow, due to his noncompliance caused by his degrading mental state. After two weeks of recurrent attempts of self removing the drainage tubes with subsequent worsening of the overall status, the case ended with exitus. Spontaneous intestinal intramural hematomas are rare complications of anticoagulant therapy. Given the patient's noncompliance and unclear history, the possibility of hematomas being post-traumatic is raising a flag in the outcomes of internal injuries.

Key words: Intestinal hematomas, perforation, mental retardation.

\section{ATYPICAL CASE OF MULTIDERMATOMAL HERPES ZOSTER IN IMMUNOCOMPETENT PATIENT}

Stoica A. Andreea' ${ }^{1}$ Madalina V.I. Doldur ${ }^{1}$, Brînzea Alice MD, PhD'

"Carol Davila" University of Medicine and Pharmacy, Bucharest, Romania.

BACKGROUND: After the primary infection with Varicella-zoster virus it becomes latent in the dorsal root ganglia and is reactivated at people with a decline in cell-mediated immunity. The reactivation causes herpes zoster which presents as a dermatomal vesicular rash, associated with severe pain. Lesions are typically unilateral, involving one dermatome and continue to form for 3 to 5 days. Among the risk factors for reactivation are advanced age, diabetes mellitus, malignancy, HIV infections, organ transplantation and immunodeficiency. CASE PRESENTATION: A case of shingles in an immunocompetent 34 years old male patient, who presented an eruption on the right flank, being extended on the back and limited by the spine. It involved multiple dermatomes and was preceded by severe pain along the affected dermatomes. The patient experienced an itching and burning sensation with intermittent stabbing pain, which was followed in 2 days by a vesicular eruption in a herpetiform pattern, on an erythematous base. The eruption started after a prolonged sun exposure in a patient with an unbalanced lifestyle. Firstly, the patient was thought to have immunodeficiency or neoplasm, but further extensive workup turned out to be negative for both of them. Diagnosed with herpes zoster, he was administrated brivudin therapy and responded well to treatment. The 
patient was discharged home with severe post herpetic neuralgia. CONCLUSION: This case illustrates the need for physicians to be extra vigilant for immunodeficiency or neoplasm in cases of young patients presenting extensive herpetic rash.

Key words: extensive herpes zoster, multidermatomes zoster, Varicellazoster virus, post herpetic neuralgia, herpetic rash.

12. ATYPICAL SEVERE WEST NILE MENINGOENCEPHALITIS. CASE REPORT Valeria N Gutuleac', Andreea-Cristina D. Stanciu², Student OanaVerginia Andronescu', Maria-Narcisa Neamtu', Mihaela Zaharia' $1 "$ Carol Davila" University of Medicine and Pharmacy, Bucharest, Romania. 2"Dr. Victor Babeș" Clinical Hospital of Infectious and Tropical Diseases, Bucharest, Romania.

BACKGROUND: West Nile Virus (WNV) has historically been known to be the least virulent viruses of the arthropod-born flaviviruses. WNV is now one of the most widely distributed arboviruses worldwide. Although $80 \%$ of infected individuals are asymptomatic, $20 \%$ develop West Nile fever, a selflimited influenza-like illness. Less than $1 \%$ of cases are represented by neuroinvasive disease, in which patients present meningitis, encephalitis and acute flaccid paralysis, as a result of direct invasion of neurons, especially those found in the brainstem, deep nuclei and anterior horn of the spinal cord. CASE PRESENTATION: We present the case of a 70-year-old woman, who was brought into our clinic, presenting fever, altered mental state and staring gaze. On clinical examination, the patient was partially cooperating, with altered temporal and spatial orientation and altered selfperception, without neurological deficits. Even more, the patient presented nuchal rigidity. The blood sample revealed leukocytosis with neutrophilia, lymphopenia, moderate microcytic hypochromic anemia. The cerebrospinal fluid (CSF) analysis showed elevated protein with positive Pandy's test, a cell count of $477 / \mathrm{mm} 3,81 \%$ being PMNs and $19 \%$ mononucleate cells. Gram stain of CSF showed no organisms. The patient was empirically started on Vancomycin and Ampicillin, which were stopped after the CSF positive results for IgM antibodies to West Nile Virus. The patient made a full recovery with no residual neurological deficits after a 14-days hospital course. CONCLUSION: In Romania, after a major outbreak in 1996, West Nile neuroinvasive disease (WNND) was reported only in a limited number of cases annually. During 2016-2017, a significant increase in the number of WNND cases was reported at the national level, associated with high mortality rates. Almost all mortality from WNV is confined to patients with neuroinvasive disease. Aging remains a dominant risk factor for susceptibility to infection with WNV and aging-associated changes in innate and adaptive immunity may contribute to increased illness among the elderly. In the present case, the West Nile infection manifested as a neuroinvasive disease which usually occurs in less than $1 \%$ of cases. Furthermore, the investigations initially performed suggested a bacterial infection rather than a viral one.

Key words: West Nile Virus, cerebrospinal fluid, neuroinvasive disease.

\section{BEARING THE FLAME: MEVALONATE KINASE DEFICIENCY}

Marc-Tudor D.E. Damian1, Bianca E. Jurjiu2, Bianca M.V. Balan3, Laura Damian MD, PhD1, Romana Vulturar3

'Emergency Cinical County Hospital, Cluj-Napoca, Romania. ${ }^{2}$ University of Oradea, Oradea, Romania. 3"Iuliu Hațieganu" University of Medicine and Pharmacy, Cluj-Napoca, Romania.

BACKGROUND: Hereditary periodic fevers are monogenic diseases belonging to autoinflammatory diseases, due to innate immune system alterations resulting in inflammatory flares. The mevalonate kinase deficiency (MVK) results in a spectrum of phenotypes, from the hyperimmunoglobulinemia $D$ syndrome manifesting with periodic fever with lymphadenopathy, to the mevalonic aciduria evolving with severe inflammation, a particular facies and cognitive deficiency. CASE PRESENTATION: A 26-year female patient presented for recurrent bouts of oral and sometimes genital aphthae, cervical lymphadenopathy, headache, disseminated arthralgia, abdominal pain and nausea, since the age of 5 . She recalled that the attacks appeared monthly, with a 5-7 duration, regressing spontaneously, but being shortened by prednisone. She was repeatedly admitted over the time and even lost a school year because of uncontrolled disease. She was suspected of familial Mediterranean fever, Behcet's disease or inflammatory bowel disease. Apart from glucocorticoids, no other therapies employed (colchicine, azathioprine, non-steroidal anti-inflammatory drugs etc) were useful. Upon presentation in a flare the laboratory revealed inflammation (erythrocyte sedimentation rate $\mathrm{mm} / \mathrm{h}, \mathrm{C}$ reactive protein $\mathrm{mg} / \mathrm{dL}$, normal $<6$ $\mathrm{mg} / \mathrm{dL}$ ) and leukocytosis (11 000/mmc) with monocytosis $(13 \%)$. The immunoglobulin $\mathrm{D}$ value was markedly elevated $(800 \mathrm{UI} / \mathrm{mL}$, normal $<100$ $\mathrm{IU} / \mathrm{mL}$ ), and IgA was also high (470, normal $70-400 \mathrm{mg} / \mathrm{dl})$. The urinary mevalonic acid was elevated. A diagnosis of mevalonate kinase deficiency was made. The mutational analysis is underway. She was started on glucocorticoids before the presumed flare start, with good clinical control. However, serum amyloid A between flares was elevated, suggesting a smoldering inflammation with systemic amyloidosis risk, therefore Colchicine was added. Efforts are being made for her to receive anti-Il-1 therapy (which is currently unavailable locally). CONCLUSION: Recurrent aphthae, cervical lymphadenopathy and digestive symptomatology may point to a mevalonate kinase deficiency. The appropriate therapy may increase the quality of life in these patients and lower the risk of systemic amyloidosis, a dreaded complication of inflammation with life-threatening potential.

Key words: mevalonate kinase deficiency, hyperimmunoglobulinemia D syndrome, aphthae.

\section{CARDIAC TRANSTHYRETIN AMYLOIDOSIS - DOES GENETIC SCREENINC TESTING OF AT-RISK ASYMPTOMATIC FAMILY MEMBERS MAKE A PROGNOSTIC DIFFERENCE?}

Diana-Ştefania V.V. Sima', Cristiana Carmen Beladan MD, $\mathrm{PhD}^{2}$ Bogdan Alexandru Popescu MD, PhD ${ }^{1,2}$

"“Carol Davila" University of Medicine and Pharmacy, Bucharest, Romania. 2"Prof. Dr. C.C. Iliescu" Emergency Institute for Cardiovascular Disease, Bucharest, Romania.

BACKGROUND: Transthyretin amyloidosis (ATTR) is a fatal disorder that is characterized primarily by progressive neuropathy and cardiomyopathy. It occurs both as a mutant form (mATTR) (with autosomal dominant inheritance) and a wild-type form (wtATTR) (predominant cardiac involvement). Deposition of amyloid into the myocardial wall causes diastolic dysfunction, restrictive physiology with late loss of systolic function, arrhythmias, conduction system disease and heart failure (HF). CASE PRESENTATION: We report the case of a 41-year old woman, with positive family history for transthyretin (TTR) gene mutation who was admitted to the hospital because of dyspnea and lower-extremity edema. The patient refused genetic screening testing 4 years ago, when her mother's sister was diagnosed with TRR gene mutation. Clinical evaluation revealed NYHA class III HF. Laboratory findings included NT- proBNP $=3845$ $\mathrm{pg} / \mathrm{ml}$ and cholestasis. Electrocardiogram showed low QRS voltage and grade I atrioventricular block and echocardiogram revealed thickened left ventricular (LV) walls, moderate LV systolic dysfunction (LV ejection fraction $=39 \%$ ), restrictive filling pattern, pericardial effusion. Electromyogram showed carpal tunnel syndrome and lower extremity sensory-motor axonal polyneuropathy. Abdominal subcutaneous fat aspiration was positive for amyloid. Further investigations included Cardiac Magnetic Resonance Imaging and Radionuclide Imaging with 99m-TC HDP (myocardial uptake of the tracer suggestive for amyloid deposits). Considering the family history and the paraclinical tests a diagnosis of mATTR was suspected and confirmed by genetic testing (Mutation E54Q). The patient was treated with loop diuretics and pharmacological therapy for ATTR was started (Tafamidis, Doxycycline and Ursodeoxycholic Acid). At 1 and 3-months follow-up the patient's clinical status and echocardiographic parameters of LV function were significantly improved (NYHA II class, no pericardial effusion, improved relaxation filling pattern). CONCLUSION: Here we report the case of a patient with hereditary ATTR (E54Q) associated with severe cardiomyopathy and neuropathy, with a favorable early response to treatment. Recent studies demonstrating greater benefit of Tafamidis in patients with less severe HF (NYHA class I and II) suggest that an early diagnosis may improve prognosis. Thus, genetic screening of at-risk asymptomatic family members of patients with hereditary ATTR can play a significant role in timely detection of the disease and initiation of disease modifying measures, thereby improving clinical outcomes.

Key words: Transthyretin amyloidosis, cardiomyopathy, diastolic dysfunction, genetic screening.

15. CASE REPORT: BOWEL OBSTRUCTION DUETO PHYTOBEZOAR IN A PARKINSON'S DISEASE PATIENT

Andrada-Ceorgiana C.C. Nacu', Munteanu M. Marius Andrei', Daniela-Tatiana Sala' 
"“Ceorge Emil Palade" University of Medicine, Pharmacy, Science and Technology of Târgu Mureș, Târgu Mureș, Romania.

BACKGROUND: A phytobezoar is a well trapped mass at the gastrointestinal level, that consists of indigestible plant material, such as fibres, skins and seeds. A phytobezoarmay generate specific symptoms: nausea, vomiting, gastric outlet obstruction, perforation, abdominal pain, bleeding. Patients who suffer from Parkinson's disease are prone to bowel obstruction, due to non-motor symptoms such as lack of peristalsis and constipation. CASE PRESENTATION: We report the case of a 70-year-old male, known with Parkinson's disease, who was admitted to the Gastroenterology Department of the Clinical County Emergency Hospital of Târgu Mureș with: severe microcytic hypochromic anemia (haemoglobin $6.2 \mathrm{~g} / \mathrm{dl}$, haematocrit $20.8 \%$ ) dehydration. During hospitalization, the patient presented bowe obstruction symptoms, and after computed tomography scan (CT-scan) a cecal tumour suspicion was raised, so he was transferred to our department for surgical treatment. We performed an exploratory laparotomy that revealed bowel obstruction due to phytobezoar (grape seeds) with ilea necrosis; we decided and practiced terminal ileum and caecum resection with termino-lateral ileocolic anastomosis. After surgery, the patient was transferred to the Intensive Care Unit. The following days we sent samples for bacteriological analysis and coagulase-negative staphylococci and Acinetobacter infection was discovered; a treatment according to the antibiogram was prescribed. Postoperative evolution was favourable therefore the patient was discharged with a good general condition, surgically cured, haemodynamically and respiratory stable and with restored bowel motion. CONCLUSION: In conclusion, we can state that patients with Parkinson's disease are more likely to develop bowe obstruction because of the autonomic features/symptoms associated with this particular pathology. The aim of this case report is to raise awareness on the importance of personalized treatment. Being such a complex and challenging condition, trying to improve quality of life is essential for these Parkinson's disease patients.

Key words: phytobezoar, Parkinson's disease, non-motor symptoms, ileum and caecum resection, personalized treatment

\section{CLINICAL ANALYSIS OF FEVER IN ASSOCIATED PATHOLOGIES}

loana I. Viziteu', Alexandru Andrei Muntean MD³, Dan-Cristian D. Munteanu², Liliana Lucia Preoțescu MD', Mircea Ioan Popa MD, PhD "'Prof. Dr. MateiBalș" National Institute of Infectious Diseases. "Carol Davila" University of Medicine and Pharmacy, Bucharest, Romania. 3 "Marius Nasta" Institute of Pneumophysiology. "“Cantacuzino" National Research Institute.

BACKGROUND: This case highlights a patient with associated pathologies along with a persistent fever which complicates a possible good evolution and response to the treatment. The challenge encountered can be observe in the need of different approach treatment and perspective of the medical team. CASE PRESENTATION: A 33 years old patient referred to the hospital of infectious diseases from a pneumology unit with a history of prolonged fever, being diagnosed with HIV infection. In the last two months he had fever almost daily accompanied by cough, loss of appetite and significan weight loss. On admission were visible scratching lesions on the legs, laterocervical microadenopathy and a right painless axillary lymphadenopathy. It was also identified pneumocystosis and a special treatment was begun, in association with HIV medication. Despite the administered antibiotics against fever, the patient felt worse and the medication needed to be changed with antifungal. A computed tomography revealed the existence of pulmonary nodules suggestive for a miliary tuberculosis and by bronchoscopy followed by sputum harvesting the diagnosis of tuberculosis has been confirmed. Pulmonary tomography shows a small number of infectious outbreaks and the treatment with firstline anti-TB drugs was started. Despite this, the patient did not respond well. He experienced pain at the hypogastric level and right axilla which has been initially painless, due to an extending tuberculosis. Lymph nodes increased so much in volume so that an extraction surgery was scheduled and the tests done after the lymphadenectomy revealed nodes full of Kochbacillus. After the intervention, the fever has been still increasing and the antifungal were replaced with antiparasitic, associating the leg lesions presents on admission with a possible parasitosis. That helped the patient to remain afebrile and a few weeks later he was discharged. Not long after due to his low immune system, he came back at the hospital because of varicella and fever. He also experienced pain again at the axilla and he needed to be reoperated, helping him otherwise to remain afebrile for more than two months. CONCLUSION: After multiple drugs combinations and a lymphadenectomy intervention, the condition of the patient improved.

Key words: fever, infection, parasitosis.

\section{CNS MIXED GERM CELL TUMOR (GCT) PERITONEAL SEEDING: CASE PRESENTATION AND LITERATURE REVIEW}

Christos G. Tsagkaris', Christos Tsitsipanis MD², Nikolaos Chochlidakis MD, PhD², Antonis Vakis ${ }^{2}$

University of Crete, Faculty of Medicine. ${ }^{2}$ University Hospital of Heraklion, Department of Neurosurgery, Heraklion, Greece.

BACKGROUND: The Central Nervous System (CNS) is the second most common manifestation location for extragonadal CCTs. CCTs account for 3 $5 \%$ of intracranial tumors in individuals younger than 20 yo and they predominantly affect men. KIT/RAS signalling or AKT1/mtor pathways associated mutations have been detected in more than $50 \%$ of all patients. Diagnostic workup includes imaging (brain and spine MRI), laboratory investigation ( $\beta$-hCG, AFP in serum and CSF) and tumor biopsy while the current treatment guidelines include radiotherapy and chemotherapy. The 5 -year survival rate of these patients has been calculated between $60-80 \%$. Peritoneal metastasis is quite rare and the existing interpretations have conceptualized the so called "seeding hypothesis". CASE PRESENTATION: A young man developed a tumor in the pineal region that grew along ventricular surfaces and caused obstructive hydrocephalus. Cytopathologic investigation demonstrated malignant cells consistent with mixed CCT. Following a ventriculoperitoneal shunt and radiotherapy - chemotherapy, the tumor and the hydrocephalus regressed. Almost ten years later, a vast abdominal - super-pubic tumor appeared.Transcutaneous biopsy specimen was diagnosed as a yolk sac tumor, a subcategory of GCTs. The patient has been referred to the surgical oncology department for further treatment. CONCLUSION: To our knowledge, 9 cases of VP shunt mediated abdomina metastasis have been reported so far. In 2 cases the abdominal tumor was identified as a yolk sac tumor with a teratoma or a germinoma as a primary tumor. A primary mixed GCT metastasizing as a yolk sac tumor has not been reported so far. Abdominal tumor development almost 10 years after the treatment of the primary tumor although the patient's VP shunt has been regularly checked. This case illustrates the need for further research on seeding and on potential prophylaxis. Taking into account the social history of the patient, this case also underlines the challenges of close cooperation between patient's family, primary care and tertiary hospital (impaired patient - residing in a rural area).

Key words: Germ cell tumor, peritoneal seeding, oncology.

\section{COMPLETE ANDROGEN INSENSITIVITY SYNDROME - CASE REPORT}

Bianca Ștefana M.C. Turcu', Ana-Maria G. Grigore', Diana-Elena G.L. Spînu', Mihaela Grigore'

'Grigore T. Popa" University of Medicine and Pharmacy, Iași, Romania.

BACKGROUND: Complete Androgen Insensitivity Syndrome (CAIS) is a type of male pseudohermaphroditism characterized by a female morphotype in individuals with $46, X Y$ karyotype and it represents an X-linked recessive disorder described for the first time by Morris. CASE PRESENTATION: We present a case of a 21-year-old woman who came for medical examination for primary amenorrhea. The patient was $165 \mathrm{~cm}$ tall and weighed $68 \mathrm{~kg}$. After the physical examination it was established Tanner stage IV breast development, absence of pubic and axillary hair and a coarse voice. The external genitalia appeared entirely female, the vagina was approximately 8-10 cm depth but it ended blindly. The ultrasound revealed that the uterus was absent and to obtain a clear status of the uterus and ovaries a computer tomography (CT) was performed. The CT also confirmed the presence of two normal sized testes into abdominal cavity near iliac vessels. After hormonal examinations serum testosterone was elevated and gonadotropins were in normal ranges. A cytogenetic analysis was also performed and it revealed that the karyotype was $46, \mathrm{XY}$. Both testes were removed using laparoscopic surgery. The pathology report showed testes with seminiferous tubules and the presence of Sertoli cells. Three other sisters of the patient with primary amenorrhea were investigated and the diagnosis was confirmed in all of them. CONCLUSION: CAIS is a disorder characterized by a female phenotype with a $46, \mathrm{XY}$ karyotype and its diagnosis and management are typically established in a multidisciplinary team specialized in disorders of sexual differentiation. It is preferred to do a gonadectomy after puberty unless there is risk of malignization. The 
genetic examination is important to find secondary cases in the same family and to accord a correct genetic counseling.

Key words: androgen insensitivity syndrome, laparoscopic gonadectomy, primary amenorrhea.

COMPLICATIONS OF A ST-ELEVATION ACUTE MYOCARDIAL INFARCTION Rebeca N. Malita', Mircea loachim Popescu'

'University of Oradea, Oradea, Romania.

BACKGROUND: ST-elevation myocardial infarction is one of the most common causes of mortality and morbidity. Survival after acute STEMI in the last time is improved, despite this mortality remains especially at high risk patients. Factors like increasing age, comorbidities can complicate the recovery and influence the survival. For these patients investigations such as electrocardiogram, chest x-ray, echocardiography and angiocoronarography are essential to discover the severity of STEMI episode. The objective of this case report is to raise awareness of a rare but possible ischemic coronary disease with a high mortality rate if no treatment is applied. CASE PRESENTATION: A 66 years old male patient presented at the Emergency department complaining chest pain, dyspnea and palpitations. Patient's medical history revealed essential arterial hypertension stage II with high cardiovascular risk, because of factors such as smoking, diabetes type II (treated with oral antihyperglycemic agents), obesity grade II, dyslipidemia and left ventricular failure. Following the clinical and paraclinical examination the patient was diagnosed with acute coronary syndrome, hypertensive cardiopathy, bivascular ischemic coronary disease (ADA, ACX) ventricular extrasystoles, intermitent atrioventricular block type Mobitz I. The EKG identified acute lower ST elevation myocardial infarction in D III and avF, with $T$ wave inversion in D I and avL. The echocardiography revealed mild mitral regurgitation with the pumping functionality preserved and hypertensive and ischemic cord appearance. The angiocoronarography carried out urgently highlights at ADA and ACX level a long stenosis (90\%). After angioplasty the patient continues to report ventricular extrasystoles. So, he underwent Holter monitoring and the results showed low ventricular arrhythmia, episodes of severe bradycardia ( 35 beats/minute) with aspect of atrioventricular block type Mobitz I and trigeminal ventricular extrasystole

thus explaining why the patient has been implanted a bicameral pacemaker ST Jude Enduring Core. The patient received treatment with acetylsalicylic acid, Ticagrelor, Atorvastatin, Pantoprazole, Amlodipine, Perindopril, Amiodarone, Metformin. The evolution was unfavourable with the following complications: heart failure, right ventricular infarction and broken ventricular free wall, interventricular septum and papillary muscles. CONCLUSION: This case presented the following particularities: the patient with heart stroke and ventricular extrasystoles had bradycardia despite undergoing the angioplasty procedure.

Key words: myocardial infarction, cardiovascular risk, mitral regurgitation, echocardiography, bradycardia, pacemaker, EKC Holter, angioplasty.

\section{DIFFICULTIES ENCOUNTERED IN THE MANAGEMENT OF CARDIAC} FAILURE IN PATIENT WITH MULTIPLE COMORBIDITIES

George N.C. Bojescu', Alice Munteanu²

"Ceorge Emil Palade" University of Medicine, Pharmacy, Science and Technology of Târgu Mureș, Târgu Mureș, Romania. 2"TituMaiorescu" University of Bucharest, Romania.

BACKGROUND: Mitral insufficiency can be caused by dilation of the left heart, often a consequence of heart failure: the left ventricle becomes enlarged and causes displacement of the attached papillary muscles, which contro the mitral valve. It is present in over $9 \%$ of people over 75 . Processes that lead to aortic insufficiency usually involve dilation of the valve annulus, thus displacing the valve leaflets. CASE PRESENTATION: A 68 years old, male patient known with arterial hypertension, dyslipidemia, insulin requiring type 2 diabetes is admitted accusing dyspnea with orthopnea and fatigability. The patient has a recent history of a triple aortic coronary bypass, left leg amputation, moderate mitral valve regurgitation and hypoxemic respiratory failure. Clinical examination reveals bilateral subcrepitant rales, rhythmic cardiac sounds, systolic murmur in the mitral area, $\mathrm{BP}=150 / 100 \mathrm{mmHg}, \mathrm{HR}=93 \mathrm{bpm}$ and no palpable peripheral pulse. The EKG presents sequelae from a previous anterior myocardial infarction, and unspecific changes of the depolarization phase in DII and aVF. CT showed cardiomegaly, minimum pericarditis and bilateral pleurisy. The spirometry reveals the patient is suffering from mixed respiratory failure with a $64 \%$ reduction of $\mathrm{FEV}_{1}$. The echography exam shows posterior mitral annular calcification, moderate LV hypertrophy, moderate LV disfunction (EF - 40\%) and type II diastolic disfunction. Considering medical investigations the patient was diagnosed with: heart failure NYHA III-IV, moderate mitral insufficiency, degenerative mild aortic insufficiency, type II diabetes, AHT gr. II, hypertensive cardiopathy, COPD, chronic respiratory failure. During hospital admission the patient received diuretic treatment and his general state improved significantly. Laboratory results came back positive for urinary infection with Klebsiella Pneumonie and the amputated area positive for Staphylococcus Haemolyticus. The patient was administered Colistin. He is undergoing treatment with: Furosemide, Spironolactone, Clopidogrel, Acetylsalicylic acid, Carvedilol, Trimetazidine, Magnesium citrate, Allopurinol, Ramipril, Amlodipine, Formoterol, Tiotropium bromide and Albuterol sulfate and has to come back for check- ups every month. CONCLUSION: The particularity of this case consists in a patient with multiple comorbidities, complex diseases and in need for increased medical care. His potentially lethal medical state imposes periodic monitoring to avert possible infections and complications.

Key words: Heart failure, Valvular failure, Chronic obstructive pulmonary disease.

\section{DOES THE ADDITION OF BITE TECHNOLOGY TO THE CLASSIC HSCT} SIGNIFICANTLY IMPROVE CLINICAL COURSE IN B-ALL?

Nicolae I.D. Andronic Gorcea', Delia Dima MD², Ciprian Tomuleasa 'Iuliu Hațieganu" University of Medicine and Pharmacy, Cluj-Napoca,

Romania. ${ }^{20}$ ncology Institute Prof. Dr. Ion Chiricuță, Cluj-Napoca, Romania.

BACKGROUND: Acute lymphoblastic leukemia (ALL) results from malignant transformation and clonal proliferation of lymphoid progenitors in the bone marrow, blood and extramedullary sites. More common in children, where cure rate reaches $80 \%$, it represents a devastating disease in adults, with only $30-40 \%$ long term remission. In recent years, novel immunotherapies like bispecific T-cell engagers (BiTEs) or chimeric antigen receptor (CAR) Tcells showed the capacity to eliminate the residual chemotherapy-resistant B-ALL cells. The only BiTE approved for clinical use is blinatumomab, a bispecific $C D 19$-directed $\mathrm{CD}_{3}$ T-cell engager that promotes the immune synapse formation. In clinical trials, it showed an increase in overall survival from 4.0 months to 7.7 months when compared with traditional chemotherapy. CASE PRESENTATION: A 24 years old man presented in June 2013 at Hematology Hospital for bilateral laterocervical adenopathies, dysphagia, cough, mild fever and weight loss for the last weeks. The final diagnosis was Philadelphia chromosome negative (Ph-) precursor B-cell acute lymphoblastic leukemia (B-ALL) with a common immunophenotypic profile. A complete remission was achieved after HyperCVAD induction chemotherapy, but he was diagnosed as MRD positive. Blinatumomab treatment led to complete molecular response and the patient followed POMP maintenance therapy until December 2016. The second relapse was treated again with HyperCVAD followed by blinatumomab and the patient acquired a second complete molecular remission, but had a persistent hypogammaglobulinemia. The patient received an allogeneic hematopoietic stem cell transplantation (alloHSCT) with consequent complete posttransplant chimerism. On July 2019 he presented with an episode of generalized seizures and a brain MRI was performed, finding lesions suggestive for recent ischemia on both precentral gyri as well as some millimetric demyelinating lesions. He was diagnosed with CNS relapse after performing a PET-CT examination and died soon thereafter. CONCLUSION: The aim of this presentation is to illustrate the therapeutic efficacy and safety of blinatumomab treatment in B-ALL and to rise clinician's attention on how to combine chemotherapy, immunotherapy and HSCT for a better disease course. It also shows that blinatumomab has hypogammaglobulinemia as a persistent adverse effect and that the isolated CNS relapse can support the idea that blinatumomab cannot penetrate the CNS.

Key words: HSCT, blinatumomab, MRD, CNS relapse.

\section{ENDOVASCULAR APPROACH OF ACUTE ISCHEMIC STROKE WITH TANDEM} OCCLUSION - CASE REPORT

Petronela Cristina P. Cristean', Olivier Vander Elst $\mathrm{MD}^{2}$, Juan Carlos Vazquez Suarez $\mathrm{MD}^{2}$, Benjamin Mine $\mathrm{MD}^{2}$, Thomas Bonnet $\mathrm{MD}^{2}$, Boris Lubicz ${ }^{2}$

"Ceorge Emil Palade" University of Medicine, Pharmacy, Science and Technology of Târgu Mureș, Târgu Mureș, Romania. ${ }^{2 H}$ ospital Erasme, Bruxelles, Belgium. 
BACKGROUND: Acute Ischemic Stroke caused by large vessel occlusion can lead to disabilitant neurological deficits by causing important cerebral lesions. In this cases, the main goal is to provide a normal blood flow as fast as possible, in order to minimize the effects of cerebral ischemia, using thrombolytic agents or endovascular techniques. Tandem occlusion is a challenging condition regarding the endovascular revascularization, requiring a combined approach with intracranial thrombectomy and extracranial stenting or angioplasty. CASE PRESENTATION: A 56 years old man presented with acute installed aphasia, palsy of right arm, inferior right facial paresis, NIHSS score $=9$. CT angiography showed a stenosis at the origin of left internal carotid artery and a decrease in caliber of middle cerebral artery. CT perfusion demonstrated a large area of hypoperfusion in the territory of the left middle cerebral artery, with a mismatch volume of $73 \mathrm{ml}$. During cerebral angiography, a $99 \%$ stenosis at the origin of left internal carotid artery and occlusion of left $\mathrm{M}_{2}$ parietal branch were observed. The angioplasty of left internal carotid artery was performed, followed by stent placement, obtaining a $90 \%$ improvement in caliber. Complete recanalization of the left MCA territory was achieved through thrombaspiration and stent retriever thrombectomy 7.5 hours after the onset of symptoms, the patient being thrombolysed 4 hours after onset. One hour post- thrombectomy non-contrast CT revealed multiple hyperdense areas in the left parieto- temporal region, which disappeared after suppression of the iodinated signal, corresponding to the destruction of the blood-brain barrier, remaining a discrete hyperdensity at the level of sylvian valley, indicating a subarachnoid hemorrhage. At 24 h non-contrast $\mathrm{CT}$, the parieto-temporal hyperdensities disappeared, persisting only the sylvian valley hyperdensity previously described, and also left parietal and superior temporal areas of recent ischemia were described. The patient was transferred to another institution with a NIHSS score of 11 and modified Rankin score of 3. CONCLUSION: According to the literature, tandem occlusions have a worse outcome than single large vessel occlusion due to the prolonged puncture-to-recanalization time. Identifying the stenotic lesion and setting the optimal endovascular strategy can lead to a faster recanalization and a better functional outcome for the patient.

Key words: Acute Ischemic Stroke, Thrombectomy, СT Perfusion, Recanalization, Endovascular.

23.

\section{HAMMAN'S SYNDROME AS PART OF THE CHEST PAIN DIFFERENTIAL DIAGNOSIS}

Nikolaos T. Kontomitros', Chalent Jamal Alexakis', Artsiom D. Klimko', Ioanna Nikoleta V. Andreopoulou', Thrasivoulos Kontomitros MD, PhD²

'Carol Davila" University of Medicine and Pharmacy, Bucharest, Romania. Internal Medicine Department, Mykonian Hygeia Polyclinic, Mykonos, Greece.

BACKGROUND: In the U.S.A alone, over 5 million patients present to the emergency department with the chief complaint of 'chest pain', the majority $(55 \%)$ of whom are found to have a noncardiac cause for their symptoms. Such an acute and rare cause is Hamman's syndrome which is characterized as spontaneous pneumomediastinum (SPM) presenting with subcutaneous emphysema. SPM is defined as the presence of interstitial air in the mediastinum without any apparent etiology. The absence of clear guidelines and the lack of results from large study groups make the management of these patients challenging. CASE PRESENTATION: A 19-year-old healthy female patient presented in our hospital's emergency department with a severe retrosternal chest pain radiating to the neck, back and left arm. The pain was aggravated by speech and deep inspiration. She also reported dyspnea, cough and numbness of the left palm and fingers. On clinical examination the patient was tachypneic with crepitation upon palpation of the neck and thoracic wall, pointing towards subcutaneous emphysema. ECG showed sinus rhythm while blood tests revealed a leukocytosis increase in $C$ - Reactive protein and negative cardiac enzymes. On chest $X$ Ray massive subcutaneous emphysema with pneumomediastinum was depicted. We performed also a chest CT-Scan in order to make sure the absence of pneumothorax and try to search for an etiology. The patient was hospitalized for 24 hours being treated with oxygen, NSAIDs, prophylactic antibiotherapy and nebulizer containing bronchodilators. Few hours after the onset of the treatment most of the patient's symptoms were diminished and in the two-week follow-up chest X-Ray there was a total resolution of the pneumomediastinum. CONCLUSION: This case underlines the diagnosis challenge of Hamman's syndrome, as it should be differentiated rapidly from other acute pathologies causing chest pain, like pericarditis, coronary syndromes, pneumothorax, aortic dissection, pulmonary embolism and Boerhaave syndrome. Thus physicians should be alert to include Hamman's syndrome in the differential diagnosis of young patients presenting to the emergency department with chest pain and take advantage of the fast information that can be gained from the clinical examination in order to have the best prognosis.

Key words: Hamman's syndrome, Pneumomediastinum, Subcutaneous emphysema, Chest pain, Pneumology.

\section{HEARING THE UNHEARD. INTRICACIES IN TREACHER COLLINS SYNDROME- CASE REPORT AND EVOLUTION IN TIME}

Alexandra-Ionela T. Strugariu', Ana-Maria C. Neagu', Irina Resmerita'

"Grigore T. Popa" University of Medicine and Pharmacy, Iași, Romania.

BACKGROUND: Treacher Collins syndrome (TCS, OMIM 154500) is a rare autosomal disorder that impairs craniofacial development by affecting the maturing of the first and second branchial arches. The estimated incidence is 1:50,000 live births. Mutations in TCOF 1 (5932) (78\%-93\%) and POLR1C or POLR1D ( $8 \%$ ) cause the disease. TCS is classically characterized by bilateral mandibular and malar hypoplasia, downward- slanting palpebral fissures, coloboma of the lower eyelid, and external ear abnormalities. About $40 \%$ $50 \%$ of individuals have conductive hearing loss attributed to malformation of the ossicles and hypoplasia of the middle ear cavities whilst, inner ear structures tend to be normal. CASE PRESENTATION: We present a 10-year-old male patient to discuss the evolution in time (starting from the age of 11 months till the age of 12 years) the frequency of clinical features, illustrating evocative and particular features, correlations between clinical signs and hearing loss. The patient is the only child of an apparently healthy, young, unrelated couple. Repeated clinical evaluations revealed: microcephaly, characteristic facial dysmorphia with bilaterally symmetric convex facia profile, downslanting palpebral fissures, depressed zygomatic arches, dental malposition, cleft palate, micro-retrognathia; deformed and lowset ears with bilateral auricular tags; delayed speech development; weight deficiency. Audiometry investigation has shown mild to severe bilateral conductive hearing loss $(\mathrm{HL})$ and both the neurological and surgical exam revealed epilepsy and hiatal hernia. CONCLUSION: The case reported is a variation of TCS. Although TCS is an rare autosomal disorder, caught in it's early stages it may ensure a life expectancy in neonates with the syndrome similar to general population. We present illustrative and particular features, as well as the importance of the multidisciplinary management approach in treatment of the patient. Early diagnosis of TCS and detection of hearring loss allows prompt and appropriate treatment of great importance for aesthetic and functional development in these patients.

Key words: hearing loss, treachercollins, tcs, tcof 1 .

\section{IMPALEMENT INJURY - CASE REPORT}

Diana-Elena G.L. Spînu', Ioana I. Chirvasa', Bianca Ștefana M.C. Turcu', Ana-Maria G. Grigore', Mircea Florin Costache MD², DanConstantin Andronic ${ }^{2}$

"Grigore T. Popa " University of Medicine and Pharmacy, Iași, Romania. ${ }^{2} 1 \mathrm{st}$ Surgical unit, Universitary Emergency Hospital "St. Spiridon" Iași, Romania.

BACKGROUND: Impalement, the penetration of the human body by using objects such as stakes, spears, hooks or poles has been documented as a torture or execution method since the beginning of recorded history. This is a successfully treated case of impalement injury. CASE PRESENTATION: We present a case of a 62-year-old man who was brought to the emergency room with a walking stick inserted into his rectum by multiple strong kicks. The tip of the stick was palpable on the right supraclavicular fossa, under the skin and the end of the stick was still out of the anal canal. The patient was hemodynamically stable and he was breathing spontaneously. The trajectory of the walking stick was established using a CT scan. Emergency laparotomy and thoracotomy have been performed and revealed the lesions of the following organs: rectum, right colon mesentery, liver, diaphragm, right lung, right second rib. Using a pendular bone saw, the stick was sectioned below the liver and the distal portion was removed transanal. The other end was pulled into the abdomen. The patient presented minimal hemoperitoneum and hemothorax which have been drained, the lung and the diaphragm have been sutured and in the transhepatic lesion we have applied Tachosil Fibrin sealant. Due to the mesocolic wounds, a right hemicolectomy has been performed and also a lateral sigmoid colostomy. 
The colostomy was reversed 3 months later. CONCLUSION: In conclusion, this type of trauma, meant to prolong the post-impalement suffering of the victim, can be survivable if the treatment is initiated immediately.

Key words: impalement, emergency, laparotomy, thoracotomy, colostomy.

\section{INCIDENTAL FINDINGS OF AN ADVANCED, INFECTED, ASYMPTOMATIC} HYDATID CYST OF A FORMER SHEPHERD

Păun F.B. Cătălin-Aurelian', Maria Silvia E. Berdeli', Stoian Silviu $M^{2}$, Bengulescu lustinian $M^{2}$

"Carol Davila" University of Medicine and Pharmacy, Bucharest, Romania.

2“Dr. Carol Davila” Clinical Nephrology Hospital, Bucharest, Romania.

BACKGROUND: The hydatid cyst is an endemic parasitic disease in our country. The parasite, Echinococcus granulosus, can only affect people that consume contaminated food. CASE PRESENTATION: A 59-yrs-old patient, exsmoker, former shepherd, admitted to the ER for trauma on his left hand underwent a chest $\mathrm{X}$-ray and the diagnosis revealed a right mediopulmonary macronodular opacity which was followed by several investigations at the pneumology clinic. The $\mathrm{CT}$ examination could not make the distinction between hydatidosis and neoplastic mass. Moreover, the investigation highlighted the presence of 2 hepatic cysts at the IVa and V segments. There was taken sputum which was tested for AARB with negative result, and lung biopsy. Despite the inflammatory aspect that underlined the possibility of hydatidosis, the investigation was not able to exclude a small cell carcinoma. In this case, the immunohistochemistry denied the presence of a tumoral pathology and supports the presence of inflammation and additionally asymptomatic infection. Ten days later, the patient is admitted to the surgery clinic for exertional dyspnoea, orthopnoea and hemoptysis. The treatment involved right superior lobectomy under general anesthesia after the evacuation of the cyst and the membrane because the lobe presented numerous supurated zones which were deemed unrecoverable. The functional status of the pacient is considered surgically improved. CONCLUSION: The case has some several points of interest, one of them being the incidental discovery of the already complicated but asymptomatic disease after assessing the patient for another pathology which concluded with late presentation to the medic. Moreover, there were problems choosing the priority in the surgical act between the lung and hepatic cysts. Nevertheless, the difficulty in the multidisciplinary assessment consisting in numerous investigations was to exclude different pathologies such as neoplasia. Also the patient will continue to require surgical treatment for the liver hydatidosis.

Key words: hydatid cyst, biopsy, lobectomy, incidental finding, asymptomatic.

27. INSIGHT INTO THE MANAGEMENT OF MULTIPLE UROTHELIAL CARCINOMA Iuliana M Stefanuca', Stefana Eugenia R. Moldovanu', Rares Constantin R. Moldovanu', Paula N. Sofica', Liviu Todosi ${ }^{1,2}$

"Grigore T. Popa " University of Medicine and Pharmacy, Iași, Romania. ${ }^{2 “ D r}$ C. I Parhon" Hospital, Department of Urology, Iași, Romania.

BACKGROUND: Urothelial tumors are tumors that can occur in the urinary tract and represent $0,03 \%$ of all cases of carcinoma. $95 \%$ of these tumors are bladder carcinoma and only $5 \%$ can be localized in the upper urinary tract. Moreover, only $1-4 \%$ are bilateral. We herein report a case of a bladder carcinoma and bilateral upper ureteral carcinoma in a patient with a single functioning right kidney. We present this case to give a deeper insight into management, treatment and prognosis of multiple urothelial carcinoma, because of the particularities of the case, it being rare. CASE PRESENTATION The patient presents with the following symptoms: recurrent hematuria biological inflammatory syndrome, lower back pain. Echography, CT and cystoscopy images gave information that helped determine a superficial tumor of the bladder, a right upper urothelial tumor and left pyelocaliceal tumor. Left renal excretion was absent. Further investigation was done and a specimen was taken using transurethral resection of bladder tumor (TURBT). The histopathological exam report revealed a highly differentiated non-invasive papillary urothelial carcinoma. After 4 weeks, the patient returned to continue therapy regarding the right upper urothelial tumor. Right segmentary lombarureterectomy and temino-terminal anastomosis on double J ureteral stent was performed, with simple secondary evolution. The patient returned 2 months later: the right double J ureteral stent was suppressed, cystoscopy control exam was performed and showed no sign of recidive regarding the bladder carcinoma. The patient then underwent left nephroureterectomy with perimeatic cystectomy for the left pyelocaliceal tumor with simple evolution. The histopathological exam revealed chronic pyelonephritis and von Brunn nests in the transversal section of the ureter. The post-operative course was uneventful. CONCLUSION: The association of a bladder carcinoma with an upper urothelial carcinoma and the bilaterality of the last mentioned is the particularity of this case. The post- operative evolution was uneventful, and the living quality of the patient was improved thanks to the good management of the case.

Key words: bladder carcinoma, upper uretheral carcinoma, double J ureteral stent, left nephroureterectomy with perimeatic cystectomy, transurethral resection of bladder tumor.

\section{LEFT VENTRICULAR NON-COMPACTION CARDIOMYOPATHY: INCIDENTAL DIAGNOSIS AFTER AN ACUTE PULMONARY EDEMA}

Adrian Gabriel C. Florescu', Ana-Maria C. Costan², Maria Magdalena Gurzun MD, PhD3, Silviu Stanciu MD, PhD ${ }^{3}$

1"George Emil Palade" University of Medicine, Pharmacy, Science and Technology of Târgu Mureș, Târgu Mureș, Romania. 2"Carol Davila" University of Medicine and Pharmacy, Bucharest, Romania. 3"Dr. Carol Davila" Central Military University Emergency Hospital Bucharest, Romania.

BACKGROUND: Left ventricular non-compaction cardiomyopathy (LVNC) is a primary genetic disorder characterized by abnormal prominent trabeculae in the left ventricle, forming deep intertrabecular recesses. The main concern resides in its complications: heart failure, thromboembolic events, malignant arrhythmias and sudden death. CASE PRESENTATION: We report the case of a 43-year-old male patient with past medical history of arterial hypertension and poor adherence to therapy who was admitted to Emergency Department with signs and symptoms suggestive of acute pulmonary edema. Patient accused dyspnea and excessive sweating. Clinical examination revealed diffuse bilateral coarse crackles consistent with pulmonary congestion, grade 3hypertension (185/100 mmHg) and discrete edema of the lower limbs. After diuretic and vasodilator treatment, patient's condition improved. The ECG examination displayed a left bundle branch block of uncertain onset with ST-T changes. Transthoracic echocardiography revealed a dilated left ventricle with severe systolic dysfunction. Considering the ECG pattern and echographic aspect, a coronary angiogram was performed which ruled out an ischemic pathology. The patient was discharged home with recommendation for further diagnostic procedures. For this purpose, the patient is admitted to The Central Military Hospital of Bucharest. Holter ECG examination displays premature ventricular complexes on sinus rhythm background. Contrast enhanced transthoracic and transesophageal echocardiography is performed and the echographic aspect is consistent with dilated subtype of LVNC, with a ratio of non-compacted to compacted layer greater than 2:1 at the end of the diastole, criteria for left ventricular dilation (End-diastolic diameter of $83 \mathrm{~mm}$, end-systolic diameter of $79 \mathrm{~mm}$ ) and systolic dysfunction (left ventricular ejection fraction of $20 \%$ ). Cardiac MRI is performed and confirms the diagnosis of LVNC. Taking into account the low ejection fraction, the Sacubitril/Valsartan $(24 \mathrm{mg} / 26 \mathrm{mg})$ therapy is initiated. Furthermore, the patient is referred to the Interventional Arrhythmology Department where undergoes a Cardiac Resynchronization Therapy. CONCLUSION: Contrast enhanced echocardiography is unquestionably a facile investigation which proves high diagnostic value in LVNC. We believe that our case report should be an incentive for the medical community to take into account more often this diagnosis, considering both the availability of the diagnostic procedures and its life- threatening yet manageable consequences.

Key words: Left ventricular non-compaction cardiomyopathy, acute pulmonary edema, contrast enhanced echocardiography.

\section{LOSING YOUR SIGHT. A RARE CASE OF DEVIC'SDISEASE}

Octavian V Baiu' Coauthors: Matei R lurea', Bianca Szabo'

'Iuliu Hațieganu" University of Medicine and Pharmacy, Cluj-Napoca, Romania.

BACKGROUND: Optic Neuromielitis (NMO) is a rare inflammatory noninfectious multifocal disease of the nervous system. It manifests as rapid onset blindness in one or both eyes, followed by varying degrees of motosensorial deficiency. The pathology is characterized by the demyelination of the optic nerves and spinal cord, lesions of the encephalus being possible in advanced stages. Optic Neuromielitis mainly affects 
women and most cases present several relapses. In the past, this disease has been considered a severe variant of multiple sclerosis (MS) because both can cause optic neuritis and myelitis. Now, the use of anti-aquaporin 4 serum antibodies have distinguished the two pathologies. CASE PRESENTATION: A 57-year-old Caucasian male presented with sudden blindness, consequently to a viral infection he experienced 3 weeks prior. ophthalmologic examination identified bilateral papilloedema, abolished photomotor reflex and absent foveal reflex. Neurological examination highlighted global, symmetrical and diminished osteotendinous reflexes and negative Babinski sign. A widening of the optic nerve sheath was found on cerebral MRI, being compatible with anterior inflammatory optic neuropathy. The patient responded well to corticosteroids, the symptoms partially disappearing. After 5 weeks from the initial attack, the patient noted a gradually increasing motor deficit, that evolved in paraparesis and paraplegia. Demyelination between T6-T10 was identified on spinal MRI and led to a diagnosis of transverse myelitis. The diagnosis of Devic's disease was based on the association between optic neuritis and acute myelitis with positivity for anti-AQP4 antibodies. Immunosuppressive therapy (methylprednisolone) and plasmapheresis led to a partial recovery of visual and neurologic functions with (measurable) increases in range of motion. CONCLUSION: While a gradual decrease in eye and motor function is expected with age, sudden blindness and sudden loss of lower limb function are uncommon. Devic's disease is diagnosed based on clinical and imagistic characteristics and also on an additional positive test for AQP4-IgC autoantibody. The acute treatment of the condition consists of glucocorticoids and plasmapheresis, while in the long term immunosuppressives and vitamin B supplements are also prescribed. Although our case is currently relapse-free, $80 \%$ of patients have recurring attacks.

Key words: Devic's disease, rapid sight loss, progressive motor deficit.

30. MALIGNANT DISEASE - CAUSE OF THE RENAL DYSFUNTION. CASE REPORT Elena-Mădălina C. Aleca', Petruța-Mădălina C. Apostol', Nadia N. Alboj', Ilie-Robert Dinu MD, PhD'

'University of Medicine and Pharmacy of Craiova, Craiova, Romania.

BACKGROUND: Multiple myeloma (MM) or Kahler's disease represents $1 \%$ of all cancers and approximately $10 \%$ of all hematologic malignancies, with an incidence in Europe of $4.5-6.0 / 100,000 /$ year. Almost all patients with MM evolve from an asymptomatic premalignant stage called monoclonal gamapathy with unspecified significance. For the diagnosis of MM there are necessary the detection and the evaluation of the monoclonal component, the evaluation of plasmocyte infiltration of the bone marrow, the evaluation of the lithic bone lesions. The presented case concerns a patient with chronic kidney disease in whom the diagnosis of the malignant disease was not easy. CASE PRESENTATION: We present the case of a 64 years-old male patient, admitted to the Emergency Department of Craiova County Clinical Emergency Hospital in March 2019 for diffuse lumbar pain with anterio irradiation, pain in the cervical-dorsal-lumbar spine, oliguria, physical asthenia and increased nitrogen retention (creatinine $=12.3 \mathrm{mg} / \mathrm{dl}$, urea $=215 \mathrm{mg} / \mathrm{dl}$ ). The patient was known having diabetes for about 4 years, hypertension with outpatient treatment. At the hospitalization, the patien presented spontaneous and movement-evoked pain of the spine, more accentuated by movements and which disappears with non-steroidal antiinflammatory drugs. Considering the important and constantly increasing nitrogen retention, a central venous catheter is mounted on the right femoral vein and the renal replacement therapy by haemodialysis is initiated. The neurological and neurosurgical interdisciplinary approach consider a lumbar polydiscopathy and recommend performing a nuclear magnetic resonance examination of the thoracic-lumbar spine. This indicates the presence of disc herniation and hydric disc degeneration on several levels. During hospitalization, a mild anemia syndrome is detected with normal erythrocyte indices and also the HBs antigen is present. Despite the painkiller treatment, the pain of the patient is not remitted and it is recommended to perform the serum protein electrophoresis that identify a monoclonal spike. The bone marrow biopsy confirms the diagnosis of multiple myeloma. CONCLUSION: Finding disc herniations and the lack of the specific bone lesions may delay the diagnosis of multiple myeloma. The renal injury which occurs in multiple myeloma may be the main reason for a patient to come to the hospital.

Key words: multiple myeloma, chronic kidney disease, polydiscopathy.

\section{MALIGNANT TRANSFORMATION OF A PANCREATIC CYST AFTER ACUTE PANCREATITIS: 15 YEARS FOLLOW-UP}

Munteanu M. Marius Andrei

Coauthors: Simona Madalina V. R. Gorga', Andrada-Georgiana C.C. $\mathrm{Nacu}^{1}$, Assoc. Prof. Simona Bota ${ }^{2}$

"George Emil Palade" University of Medicine, Pharmacy, Science and Technology of Târgu Mureș, Târgu Mureș, Romania. ${ }^{2}$ AbteilungfürInnereMedizin und Gastroenterologie, Hepatologie, $\begin{array}{ll}\text { Endokrinologie, } & \text { Rheumatologie } \\ \text { NephrologiemitzentrallerNotfallaufnahme (IMuC); Klinikum Klagenfurt am }\end{array}$ Wörthersee.

BACKGROUND: Our aim is to present the malignant transformation of a benign pancreatic cyst and showing the importance of follow-up investigations in similar cases. CASE PRESENTATION: 61-year-old female patient was admitted in $03 / 2018$ because of abdominal pain. She was treated in 2003 for acute pancreatitis. At that time Computed Tomography (CT) showed a $2.2 \mathrm{~cm}$ necrosis of the pancreatic cauda and peripancreatic infiltrates. The cystic lesion increased to $12 \mathrm{~cm}$. The Endoscopic Retrograde Cholangiopancreatography (ERCP) has excluded a pancreatic duct fistula. An Endoscopic Ultrasound (EUS) with pigtail drainage was carried out. Stent was removed one month later after cyst resolution. In 2007, the pancreatic cyst recurred and grew up to $12 \mathrm{~cm}$ until 2010. The patient remained asymptomatic until 2018 (2018: cyst size: $20 \mathrm{~cm}$ and patient presents abdominal pain). EUS drainage with plastic stent was performed. One day after the intervention, there was a marked increase in C-Reactive Protein levels. The CT showed free air and fluid. A surgery was performed showing a dislocated stent. The perforation site was closed but the supposedly benign cyst was not removed because of the massive pericystic collateral circulation. The patient was resumed in $05 / 2018$ due to abdominal pain. EUS drainage with a Hot AXIOS ${ }^{\circ}$ stent was performed. Three days after the procedure, one Haemoglobin drop was observed. The CT showed a $5 \mathrm{~mm}$ pseudoaneurysm. The angiography showed no active bleeding. The metal stent could be removed after 4 weeks. After removal of the stent, the patient presented fever and again suspicion of an infected cyst. The ERCP showed communication between the cyst and the pancreatic duct (plastic stent in the pancreatic duct was placed). After another unsuccessful attempt at external drainage, surgery was performed. Histology showed a moderately differentiated invasive intraductal papillary- mucinous adenocarcinoma. CONCLUSION: Malignant transformation of pancreatic cysts after acute pancreatitis is possible.

Key words: Malignant transformation, cyst, acute pancreatitis.

\section{METASTATIC COLORECTAL CANCER - AN UNEXPECTED JOURNEY}

Gabriel D. Ceapa', Elena-Maria M. Capatina', Iaciu Cristian MD, PhD ${ }^{2}$ "Carol Davila" University of Medicine and Pharmacy, Bucharest, Romania. ${ }^{2}$ "Elias” University Emergency Hospital, Bucharest, Romania.

BACKGROUND: Colorectal cancer (CRC) is a major morbi-mortality cause yearly there are 1,2 million cases diagnosed and over 600.000 deaths reported. Known risk factors are: age $>40$, personal or familial history of adenomatous polyps, inflammatory bowel disease, CRC; "Western" diet; smoking and alcohol consumption. $80 \%$ of all CRC are caused by acquired genetic defects; the most frequent oncogenes are: KRAS, BRAF, MYC and tumor-suppressor genes. For CRC the 5 -year survival rate is $64 \%$; for rectal cancer, $67 \%$. If spread, the survival rate drops to $14 \%$ for CRC and $15 \%$ for rectal cancer. CASE PRESENTATION: 2015: male patient D.P., aged 64, presenting with subocclusive syndrome undergoes a chest-abdomen-pelvis CT scan with contrast showing a malignant stenosing infiltrative process, affecting $77 \mathrm{~mm}$ in the rectosigmoid junction with multiple hepatic and vertebral metastases. At colonoscopy the primary tumor is discovered, 25 $\mathrm{cm}$ from the anal orifice. Clinical status: ECOG 1 . The tumour is an intestinal type ADK, mutant RAS gene-positive. FOLFOX VI+ Avastin Palliative Chemotherapy ( $\mathrm{PCT} 1$ ) treatment is initiated, with mixed oncological response- $\mathrm{M}_{1}$ OSS continue spreading over the next year and a half. PCT1 causes mixed polyneuropathy; the treatment is changed to 5 - FU, Leucovorin and Avastin (PCT2)- August 2016. The response to PCT2 after one yea continues to be mixed: $M_{1}$ hepatic in progression, $M_{1}$ OSS in remission. 2017: Slightly progressive disease, ECOC status 1; surgery is not recommended. 2018: Imaging proves the progression of all metastases. PCT2 continues. 2019: Due to the progression of all metastases FOLFOX VI treatment is reinitiated and a biopsy is acquired from M1 OSS. May: RASwild type status. July: Oncological disease in progression. Status ECOC 1 PCT3 initiated: Cetuximab+ FOLFIRI. CONCLUSION: This case presents multiple unexpected changes in evolution; The maintained ECOG 1 status from 
diagnosis to the present day, and defying the life expectancy associated with his IV-stage CRC diagnosis. Although initially testing positive for RASmutation, through his treatment mutations switched, and is currently classified as a RAS-wild type CRC patient. This raises many questions such as what the future of both his treatment and overall life expectancy could be.

Key words: Colorectal cancer, Oncology, RAS, mutant RAS, RAS-wild type, KRAS, metastatic colorectal cancer, CRC.

\section{NOVEL MULTIMODAL APPROACH FOR MALIGNANT MELANOMA IN THE METASTATIC SETTING}

Elena L. Juganaru', Mihai-Teodor Georgescu

"Carol Davila" University of Medicine and Pharmacy, Bucharest, Romania.

BACKGROUND: Malignant melanoma (MM) is the least common but the deadliest skin cancer, with about 9,000 victims each year. Almost $20 \%$ of all MM patients develop brain metastases (BM), for whom few options have been available prior to 2010. Although antibodies targeting the programmed cell death protein-1(antiPD-1) have been recently introduced into practice, this disease still remains a huge therapeutic challenge in the metastatic stage. CASE PRESENTATION: A 57-year-old male presented with a $3 / 3 \mathrm{~cm}$ left eyebrow pigmented lesion for which local excision was performed. The pathology exam confirmed the MM diagnosis, Clark index IV, Breslow index $2,5 \mathrm{~mm}$, free of lympho- vascular and perineural invasion, 70 mitosis index and a $\mathrm{Ki} 67$ of $70 \%$. Postoperative cranial computer tomography (CT) was consistent with foreign body granuloma. Therefore, pericicatricial reexcision was performed with no evidence of local relapse. On a subsequent followup 9 months later, whole body CT scan revealed an iodophilic nodular lesion in the left parotid gland and periglandular enlarged lymph nodes suggesting a lymph node relapse. Punch-biopsy confirmed the MM origin of the cells. BRAF V600 testing was negative for mutations. The patient was noted to have a low performance status, left-sided paresis, visual and verbal impairment. A brain magnetic resonance imaging (MRI) identified 6 BM with perilesional edema. The multidisciplinary oncological board decided for a modern multimodal treatment approach. Whole-brain radiotherapy up to 36Cy was performed resulting in a complete regression of 5 of the lesions and a persistent pontine metastasis, which was surgically removed. Also, treatment with Nivolumab (anti PD-1 antibody) has been initiated, using a $240 \mathrm{mg} / \mathrm{sqm}$ dosage, days 1 and 15, 93w. Three years after, at the latest follow-up, whole body CT scan and brain MRI show no signs of disease progression and a regression of the parotid gland tumor. Nivolumab administration has not been interrupted, the patient currently undergoing the 25th treatment cycle. CONCLUSION: The objective of this case report is to highlight the magnitude of the potential benefits we can expect against metastatic MM as therapeutic options have consistently developed lately. AntiPD-1 antibodies are emerging as new promising treatments with a significant improvement of the survival rate of these patients.

Key words: Nivolumab, AntiPD-1 Antibodies, Brain Metastases.

34. PALLISTER-KILLIAN SYNDROME: AN UNACKNOWLEDGED CAUSE OF EPILEPSY

Andreea Florentina D. Șerban' Coauthors: Rodica-Iulia Ch. Iorga', Budișteanu Magdalena

1"Carol Davila" University of Medicine and Pharmacy, Bucharest, Romania. 2"Prof. Dr. Alexandru Obregia" Clinical Hospital of Psychiatry, Bucharest, Romania.

BACKGROUND: Pallister-Killian syndrome (PKS) is a rare genetic disorder caused by tetrasomy $12 \mathrm{p}$ mosaicism associated with a supernumerary isochromosome. Although the clinical manifestations of PKS are variable, it is most common characterized by craniofacial dysmorphism, learning disability and epilepsy. A significant number of patients will develop a seizure disorder, mostly starting in early infancy. However, seizure and epilepsy characteristics of people with PKS are poorly described. CASE PRESENTATION: A 6-month-old girl was referred to the department of Pediatric Neurology for 2 episodes of seizures which resembled spasms, no head control, inability to grasp objects, she says no syllables. She was born at 36 weeks, through cesarean delivery, after a normal pregnancy until week 24 when polyhydramnios was revealed in fetal ultrasound. Anthropometry showed normal weight $=2840 \mathrm{~g}$, birth length $=49 \mathrm{~cm}$ and head circumference $=36 \mathrm{~cm}$. APGAR scores were $4 / 1^{\prime}, 6 / 5^{\prime}, 8 / 10^{\prime}$, therefore positive- pressure ventilation was initiated, followed by oxygen therapy in the incubator. At the age of 5 months, an antiepileptic treatment with valproate was recommended, with good response. Physical examination showed: height $=50 \mathrm{~cm}$ ( $\mathrm{PC}$ 75), weight $=6,2 \mathrm{~kg}$ ( $P C$ 25), head circumference $=43 \mathrm{~cm}(P C 75)$; a hypopigmented patch about $2 \mathrm{~cm}$ on right ankle joint, mediooccipital hemangioma, a hyperpigmented patch on left lumbar region and bilateral frontal alopecia; a distinctive facial appearance, including bulging forehead, low set posteriorly rotated ears, bilateral partial palpebral ptosis, sparseness of eyebrows, broad nasal bridge, wide mouth with thin upper lip and no teeth; short, conical hand fingers and high set flexed hallux. Neurological examination revealed delayed psychomotor development: no head control, at vertical suspension she cannot support her weight; generalized hypotonia; mental age of about 2 months. She started babbling at 3-4 months old. Cerebral MRI showed no modifications. EEG displayed theta background activity with medium voltage, without aspects of hypsarrhythmia. Cenetic tests, array-based comparative genomic hybridization (array CGH) and FISH with a specifically probe for $12 \mathrm{p}$, revealed tetrasomy 12p mosaicism, which confirmed our suspicion. CONCLUSION: PKS is a rare genetic cause of intellectual disability and epilepsy in children. Characteristics of epilepsy in PKS are yet to be discovered, but PKS should be considered in the differential diagnosis of epilepsy.

Key words: Pallister-Killian syndrome, epilepsy, intellectual disability.

\section{PERMANENT DIABETES INSIPIDUS AND TRAUMATIC BRAIN INJURY: IS THERE A LINK?}

Ana-Maria S. Cheorghe' Coauthors: Adriana V. Ion', Iulia Soare², Lect. Sorina Martin ${ }^{1,2}$

"Carol Davila" University of Medicine and Pharmacy, Bucharest, Romania. 2"Elias" University Emergency Hospital, Bucharest, Romania.

BACKGROUND: Central diabetes insipidus is the most common form-due to low ADH secretion by posterior pituitary. It is a condition characterized by polyuria with hypotonic urine accompanied by polydipsia. Although traumatic brain injury (TBI) can often lead to hypopituitarism, posttraumatic diabetes insipidus becomes permanent in a minority of cases. We chose this topic because the impairment of ADH secretion following a cranial trauma and maintenance of diabetes insipidus (DI) for a long time is rather rare. Prompt diagnosis and management can decrease the mortality and morbidity associated with this condition. CASE PRESENTATION: A 31-year-old male, smoker of 12 pack-year, was admitted to the Endocrinology Department, in August 2019 for polydipsia, polyuria, nicturia, frontal headache and dizziness. The onset of symptoms was four years earlier after he suffered a car crash followed by temporary amnesia. Physical examination revealed a diuresis of $3-5 \mathrm{~L}$ accompanied by nicturia (1-2 micturitions/night), normal blood pressure. General tests showed normal glycemia and natremia, with low urinary density (1010). Given the TBI history, the patient had complete pituitary function tested. Hormone tests (TSH, ACTH, prolactin, FSH, ICF1, LH) revealed no dysfunction of the anterior pituitary. The patient underwent a water deprivation test. The results were highly suggestive for central diabetes insipidus. Pituitary MRI showed homogeneous signal of adenohypophysis, but a lack of the $\mathrm{T}_{1}$ signal hyperintensity characteristic for neurohypophysis, suggesting the lack of $\mathrm{ADH}$ and supporting the diagnosis of central diabetes insipidus, post TBI. The patient was prescribed desmopressin $120 \mu \mathrm{g} /$ day, with immediate improvement of the symptoms. CONCLUSION: While TBI can lead to hypopituitarism, it is a rare cause of DI. This case is particular because of the presence of permanent post traumatic DI, without adenohypophyseal damage. It also underlines the importance of neurohypophyseal assessment following traumatic brain injury, even in the absence of other anterior insufficiencies. Long term and close follow up is required in these cases.

Key words: diabetes insipidus, pituitary insufficiency, brain injury, ADH.

\section{POST TRABECULECTOMY CHOROIDAL EFFUSION IN A YOUNG PATIENT WITH STURGE WEBER SYNDROME}

Beatrice C. Balaceanu' Coauthors: Atanas Bogoev², H. Burkhard Dick ${ }^{3}$ 'Vision Eye Clinic 2"Carol Davila" University of Medicine and Pharmacy, Bucharest, Romania. ${ }^{3}$ Ruhr Universität Bochum, University Eye Clinic Bochum, Germany.

BACKGROUND: Sturge Weber Syndrome is a rare neurocutaneous disorder associated with the somatic mutation of the GNAQ gene. This condition is characterized by three major features: a port-wine birthmark caused by the hamartomatous vascular proliferation, an ipsilateral leptomeningeal 
angioma and glaucoma (increased intraocular pressure), leading to various degrees of neurological and ocular abnormalities. The increased intraocular pressure typically develops in childhood or early adulthood, and is rated as the most frequent ocular comorbidity. It is generally characterized by unresponsiveness to standard treatment and the severe visual impairment at the moment of the referral. CASE PRESENTATION: A 16-year-old female patient presented to the 0phthalmology Department with a history of unilateral increased intraocular pressure due to secondary glaucoma and nevus flammeus associated with Sturge Weber Syndrome. She underwen trabeculectomy of the left eye in February 2019 and has been unde medication with prednisolone eye drops ( 2 times/day) in the left eye and brimonidine eye drops ( 2 times/day) and bimatoprost eye drops ( time/day) in the right eye. Examination of right eye showed an enlarged globe, increased conjunctival vascularization, episcleral vessel dilatation along with a decreased visual acuity of the right eye with an intraocula pressure (IOP) of $27 \mathrm{mmHg}$. She was scheduled for a right eye trabeculectomy under a general anesthesia in order to obtain long-term control of the intraocular pressure. The postoperative period was complicated with a choroidal effusion syndrome and a IOP of $32 \mathrm{mmH}$ demanding following treatment with topical ofloxacin, atropine and prednisolone. CONCLUSION: Sturge Weber syndrome demands a multidisciplinary approach and when referring to glaucoma management, surgery is considered both necessary and challenging. When performing ocular surgery on such cases the surgeon should be prepared to deal with the increased chance of complications: choroidal effusion, expulsive hemorrhage and exudative retinal detachment. It is however crucial to plan an early postoperative ophthalmic examination and treatment of the complications as soon as possible for the best outcome.

Key words: sturge weber syndrome, trabeculectomy, choroidal effusion.

\section{RARE ASSOCIATION OF NEOPLASTIC DISEASES- BEING BLINDED BY ONE DIAGNOSIS}

Anamaria Romana V. L. Orghidan', Cristian Velicescu ${ }^{1,2}$

"Grigore T. Popa " University of Medicine and Pharmacy, lasi, Romania.

2"St.Spiridon" Emergency Hospital Iași.

BACKGROUND: Neoplastic diseases represent the pathology with the highes morbidity and mortality rates, mainly because of the late diagnosis. In the majority of cases the patients are admitted into the hospital with advanced or metastatic stages of cancer. Associations of different types of malignant tumors are reported in the medical literature. When this occurs, the symptomatology of some neoplasms can be masked by other existing diseases - as in the case of our patient. CASE PRESENTATION: We are presenting the case of a 72 yeas old male admitted into the "Saint Spiridon" Ceneral Surgery Clinic with giant cervico-mediastinal goiter that caused tracheal and esophageal compressive symptoms- dysphagia for solid foods and acute respiratory insufficiency. The patient was previously investigated in the endocrinology clinic. Upon admission the patient presented tegument pallor, dyspnea, increased respiratory effort and dysphagia for solid foods. The blood tests show that the patient has a very low hemoglobin level of $5.8 \mathrm{~g} / \mathrm{dL}$, indicating severe anemia-that was, however, well tolerated. Also he was positive for fecal occult blood indicating gastrointestinal bleeding. Endoscopy and diagnostic colonoscopy were performed and the latte showed a bleeding, circumferential, vegetative mass located in the caecum. The biopsy revealed a moderately differentiated colon adenocarcinoma. After preoperative preparation and hemodynamic stabilization of the patient, total thyroidectomy was performed and during the same operative session right hemicolectomy with latero-lateral ileotransverseanastomosys were performed. The pathology examination results confirmed the presence of the colon adenocarcinoma (T3NoMo) and a thyroid with giant anizofollicular goiter without tumoral growths. CONCLUSION: The patient was hospitalised for an endocrine disease with compressive symptoms on both trachea and esophagus. However, the much severe, advanced condition, that would predict the prognosis, was not diagnosed previously to hospitalisation in our clinic. It is of major importance not to be blinded by one diagnosis but be able to detect the distinct diseases that the patient suffers from.

Key words: caecum, neoplasm, giant goiter.

\section{RELAPSING POLYCHONDRITIS - A CHALLENGING DIAGNOSIS}

Ioana-Irina C. Rezus' ${ }^{1}$, Celina 0 Luca1, Andreea F. Ciolacu', Elena Rezus $^{1,2}$
'"Grigore T. Popa " University of Medicine and Pharmacy, Iasi, Romania.

${ }^{2}$ Rheumatology Clinic, Clinical Rehabilitation Hospital, Iasi, Romania.

BACKGROUND: Relapsing polychondritis is a rare autoimmune disease of unknown etiology with episodic but potentially progressive inflammatory manifestations that affect both cartilaginous and noncartilaginous structures and can be accompanied by severe pulmonary complications. The clinical features include inflammation of the auricular, nasal, laryngotracheal, costal, articular cartilage and ocular and inner ear inflammation. CASE PRESENTATION: We present the case of a patient, M.C., 46, female, who has been admitted to the Rheumatology Clinic of the Rehabilitation Hospital lasi for pain and swelling of the right auricular cartilage and nasal cartilage. She had been suffering from relapsing inflammatory episodes for approximately 2 years. During the clinical examination we observed a deformed, swollen, painful right pinna, with the aspect of the "cauliflower ear", a swollen left pinna and a nose deformity due to the nasal cartilage inflammation. Our paraclinical examinations included blood tests, X-ray examinations, electrocardiography and echocardiography. There were no significant changes of the inflammatory markers and the number of the total antinuclear antibodies and the levels of the rheumatoid factor were within normal. The skull X-ray presented a saddle-nose deformity. The chest $X$-ray showed no signs of respiratory disease and the heart investigations did not show any signs of cardiovascular diseases. We performed a biopsy of the right auricular cartilage which showed an elastic auricular cartilage with a fibrous reshaping area that also affected the perichondrium in the central area, confirming the auricular chondritis. We initiated a treatment with Methotrexate, subcutaneously, $15 \mathrm{mg}$ per week for the first four weeks followed by a continuous life- long treatment of $20 \mathrm{mg}$ per week. The clinical evolution was favorable. CONCLUSION: Although the diagnosis of relapsing polychondritis is relatively easy, many conditions can mimic the initial changes, therefore clinicians need to pay much attention to all the existing signs and symptoms in order to do a correct and complete clinical examination. With no specific laboratory findings, a clinical polymorphism resulted from a real bazaar of manifestations and episodic occurrence, the final diagnosis can be significantly delayed.

Key words: Cauliflower ear, inflammation, chondritis.

39.

\section{RENAL ARTERY STENOSIS: EXTREME THERAPEUTIC MEASURES FOR A} CRITICAL CLINICAL SITUATION

Delia Melania I. Popa', Mara-Lucia G. Moraru', Alexandru-Dan R. C. Costache1, Nicoleta M. Dumitrescu', Daniel Dăscălescu MD, Nicușor Lovin MD, Larisa Anghel', Cristian Stătescu', Liviu Macovei', Mircea Balasanian'

'"Grigore T. Popa" University of Medicine and Pharmacy, Iași, Romania.

BACKGROUND: Renal artery stenosis is the leading cause of renovascular hypertension, being responsible for about $10 \%$ of severe refractory arterial hypertension cases, some of them often complicated by acute pulmonary edema (APE). CASE PRESENTATION: We are presenting the clinical case of a 64-year-old female patient, known with important cardiovascular pathology: chronic coronary artery disease, complete right renal artery occlusion and $60 \%$ left renal artery stenosis, documented by direct angiography and computed tomography (CT) angiography, secondary 3 rd degree arterial hypertension, with multiple episodes of APE. She also associated renal (chronic renal disease) and neurological (left temporal-parietal ischemic stroke) pathologies. The patient was admitted at the "Dr. C.I. Parhon" Clinical Hospital Nephrology Clinic lasi for the acute exacerbation of chronic kidney disease and APE associated with a systolic blood pressure value of $210 \mathrm{mmHg}$. She was therefore redirected to the „Prof. Dr. Ceorge I.M. Georgescu" Institute Cardiology Clinic in order to establish the opportunity for stenting the lesion on the left renal artery and the subsequent therapeutic conduct. Due to multiple episodes of APE during hospitalization and dyspnea with quasi-permanent orthopnea, the decision of endotracheal intubation and mechanically assisted ventilation was made in order to facilitate the selective renal artery angiography, which showed $95 \%$ stenosis of the left renal artery and complete occlusion of the right renal artery. Angioplasty and stenting of the left renal artery was performed, resulting in a sharp decrease in blood pressure, followed by a significant reduction of antihypertensive medication and improvement of the renal function. CONCLUSION: This case illustrates that the decision regarding renal artery angioplasty in renovascular hypertension should be based on the individua characteristics of the patient, such as life expectancy, presence of comorbidities, quality of blood pressure control and status of renal function. 
Although evidence that shows the benefit of choosing interventional treatment instead of medication is not clear enough, we can accept that in patients with particular clinical scenarios, with anatomically and functionally significant renal artery stenosis, deterioration of renal function and / or repeated hospitalizations for APE, renal artery angioplasty could have substantial benefits.

Key words: renal artery stenosis, renovascular hypertension, angioplasty, stenting.

\section{SACROCOCCYGEAL TERATOMA - FROM PRENATAL DIAGNOSIS UNTIL} THERAPEUTIC PROCEDURE. CASE REPORT

Nadia N. Alboj', Petruța-Mădălina G. Apostol', Elena-Mădălina C. Aleca', Alin Stoica'

'University of Medicine and Pharmacy of Craiova, Craiova,Romania.

BACKGROUND: A teratoma is a rare type of congenital germ cell tumor that can contain fully developed tissues and organs, including hair, teeth, muscles and bone. The sacrococcygeal teratoma (SCT) is one that develops in the coccyx or tailbone and it is the most common tumor found in newborns and children, but it is still rare overall. It occurs in about 1 in every 35,000 to 40,000 infants and it is more often in females than males (4:1). CASE PRESENTATION: We present a case of a female newborn which was diagnosed by a routine prenatal ultrasound examination with sacrococcygeal tumor. At birth the patient presented a firm tumoral mass of $9 / 9$ centimeters and clubfoot: varusequin. Although this type of teratoma is frequently associated with various malformations, we couldn't find a link between varusequin and SCT. The patient didn't present any other malformations. CONCLUSION: A surgery was performed to remove the tumor which occupied the space between the coccyx and rectum, the right gluteal region, beyond the median line to the left gluteal region. The teratoma was well delimited and resected in toto with the tip of the coccyx. Ligation of the median sacral artery was performed. The tumor was completely removed and the result was pleasing. Although the sacrococcygeal teratoma is a rare condition and the cause of it is unknown, it can be diagnosed early during the intrauterine life and the surgery is always required after birth as the tumor may cause genitourinary and gastrointestinal symptoms even though it is benign. Even if most of these tumors are benign, they may grow quite large and many will become malignant if surgical resection is not performed.

Key words: Sacrococcygeal teratoma, Malformation, Prenatal diagnosis.

\section{SEVERE CONGENITAL CANDIDIASIS LEADING TO DEATH IN A PRETERM} INFANT

Iustina I.A. Grosu', Maria Irina M. Fediuc ${ }^{1}$, George Simion MD', Tiberiu Augustin Ceorgescu', Maria Sajin'

"Carol Davila" University of Medicine and Pharmacy, Bucharest, Romania.

BACKGROUND: Congenital candidiasis is a rare and severe infection contracted in utero and manifested within the first 6 days of life, which affects predominantly premature and low birth weight newborns. It occurs in less than $1 \%$ of women infected with Candida albicans during pregnancy and can be either skin-localized, with generalized maculopapular eruptions (which result in extensive desquamation), or systemic, leading to respiratory distress, hepatosplenomegaly, meningitis, sepsis and death. If detected early, congenital candidiasis is rarely fatal, but due to its low incidence and non-specific symptoms, correct diagnosis might be delayed. CASE PRESENTATION: We present the case of a 23-year-old primigravida with an uninvestigated pregnancy at 30 weeks of gestation, who was admitted at the Emergency University Hospital in Bucharest due to leucorrhea and abdominal pain. During hospital stay, the mother presented uterine contractions with spontaneous rupture of membranes and delivered a male infant weighing $1100 \mathrm{~g}$ with an Apgar score of 9 . Shortly after birth, the fetus developed fever and respiratory distress and died after 76 hours due to multiorgan failure. Fetal autopsy and subsequent histopathological evaluation revealed angioinvasive hyphae in the lung, brain, liver, spleen, kidney, striated muscle and gastrointestinal tract, which stained positive with Periodic acid-Schiff and Grocott's methenamine silver. Cultures in Sabouraund agar confirmed Candida species. CONCLUSION: Congenital candidiasis is a rare yet serious condition, which should be considered in the differential diagnosis of generalized maculopapular or pustular skin eruptions in neonates, alongside with Listeria monocytogenes infection, chickenpox, herpes virus infection or syphilis. Major risk factors, such as low birth weight, prior use of antibiotics, central venous catheters or parenteral alimentation should also be taken into consideration. Early recognition and prompt treatment implementation of antifungal agents are important as they increase the infant's chances of recovery with minimal repercussions.

Key words: Congenital candidiasis, premature infant.

\section{THE DIAGNOSTIC CHALLENGES IN OSTEOARTICULAR TUBERCULOSIS}

Marchis Hund G.0. Elisabeth Antonia', Pelea Michael MD', Prof. Fodor Daniela'

"Iuliu Hațieganu" University of Medicine and Pharmacy, Cluj-Napoca, Romania.

BACKGROUND: Tuberculosis (TB) is an endemic disease in developing countries, but the prevalence is rising all around the world. Broadening immunosuppressive conditions and enormous migrations are the main pillars of this growth. In the extrapulmonary forms of tuberculosis, skeletal situses account for 10 to $35 \%$ of cases. CASE PRESENTATION: In 2016 a 48 years old male patient was admitted to the hospital for purulent expectoration, heavy perspiration and fever. The Rx examination found a nodular infiltrative opacity in the superior left pulmonary lobe. Mycobacterium tuberculosis was identified as negative, and treatment with Ceftriaxone, Ciprofloxacin, and Centamicin was prescribed. The patient reacted with a bilateral swelling of his knees and only the right knee recovered through the interruption of treatment. After several pulmonary reinfections, highh-intensity knee pain arose in association with pretibial and plantar erythema nodosum. The diagnosis of a Wegener vasculitis was established after Anti-Ro 52 and Quantiferon were positive, and Prednisone alleviated the symptoms. After the interruption of the treatment symptoms of fever, nocturne perspiration, erythema nodosum reappeared and a viscous liquid with negative bacteriology, no crystals, was evacuated from the painful knee. Shortly the patient developed a baker cyst, which led to a deep vein thrombosis. Arthroscopic synovectomy identified a granulomatous synovitis, without central necrosis and rare Langerhans cells. In 2019 the left knee became swollen and painful again. Ultrasound examination showed the proliferation of the synovia, liquid in the articulation, erosion of the condyles and significant fibrosis with a tightened articular space. The AFB smear was negative, but ANA antibodies were positive. After 5 weeks the Mycobacterium Tuberculosis culture established the final diagnosis of osteoarticular tuberculosis. CONCLUSION: The pulmonary origin could not be identified, so the suspicion of primary joint tuberculosis is the most probable, even though it is a rare condition that is usually present in children. Diagnosing osteoarticular TB still represents a challenge because microbiological investigations are frequently negative and misdiagnosis leads to irreversible consequences.

Key words: Tuberculosis, Skeletal, Chronic.

\section{THE EISENMENGER SYNDROME - A PIECE OF CAKE?}

Andreea Alexandra C. Anghel', Ilknur Nihal Nurittin Ardic ${ }^{2}$, Ozgur Bayturan MD³

'Ankara University Medical School. 2"Iuliu Hațieganu" University of Medicine and Pharmacy, Cluj-Napoca, Romania. ${ }^{3}$ Celal Bayar University School of Medicine, Manisa, Turkey.

BACKGROUND: Ventricular septal defects (VSD), atrial septal defects (ASD) and patent ductus arteriosus (PDA) are only a few of the congenital cardiac abnormalities associated with development of the Eisenmenger syndrome. Due to their tendency of spontaneously closing during childhood, VSD represent only $10 \%$ of adult congenital heart defects. Such patients face the risk of developing pulmonary vascular disease, followed by the Eisenmenger Syndrome. CASE PRESENTATION: A 28-year-old female patient sought medical assistance in the emergency department of Manisa 8 Eylul Hospital due to persistent cough and insomnia. Cardiac arrest shortly developed in the same department, with successful cardio-pulmonary resuscitation and full recovery. The patient refused the recommended echocardiography and was discharged after two days from intensive-care unit. The suspicion of Eisenmenger syndrome and myocarditis was raised. Four days later, she consulted the emergency service in our hospital for pricking chest pain and dyspnea. Echocardiography showed serious tricuspid insufficiency, right ventricular hypertrophy, pulmonary hypertension and a $2.4 \mathrm{~cm}$ nonrestrictive perimembranous VSD (membranous defect extended to the muscular septum). This, together with the central cyanosis and pulmonary 
hypertension, allowed the diagnosis of Eisenmenger syndrome. Oxygen therapy was started, together with antibiotic therapy, as the patient developed pneumonia. Surgical intervention was no longer an option. CONCLUSION: Survival in Eisenmenger complex patients is limited and quality of life decreased. Moreover, patients face the risk of endocarditis, aortic regurgitation, sinus of Valsalva aneurysm, arrhythmias and pulmonary infections. We conclude that congenital VSDs should be the object of periodical follow up until their closure, and that all adult patients should be referred to a congenital heart disease expert, who can recommend surgical intervention in time to avoid irreversible complications.

Key words: Eisenmenger syndrome, perimembranous ventricular septal deffect, pulmonary vascular disease.

44. THE IMPORTANCE OF ENDOSCOPIC ULTRASOUND IN ESTABLISHING THE CORRECT DIAGNOSIS - A CASE SERIES

Simona Madalina V. R. Gorga', Munteanu M. Marius Andrei', Marcel Razpotnik $\mathrm{MD}^{2}$, Simona Bota ${ }^{2}$

"George Emil Palade" University of Medicine, Pharmacy, Science and Technology of Târgu Mureș, Târgu Mureș, Romania. 2AbteilungfürlnnereMedizin und Gastroenterologie, Hepatologie, Endokrinologie, Rheumatologie und NephrologiemitzentrallerNotfallaufnahme (IMuC); Klinikum Klagenfurt am Wörthersee.

BACKGROUND: The aim of this paper is to present how Endoscopic Ultrasound (EUS) was decisive in establishing the correct diagnosis in a series of patients. Cases where EUS established the correct diagnosis despite inconclusive radiologic evaluation, were identified in our EUS database. CASE PRESENTATION: Case 1 - 74-year old female patient, hospitalized due to acute pancreatitis of unknown etiology. Computed Tomography (CT) Scan showed a normal pancreas. EUS excluded a biliary etiology, but a $1.7 \times 1.5 \mathrm{~cm}$ hypoechoic mass in the head of the pancreas was identified. Magnetic Resonance Imaging was performed but, could not clearly detect the lesion. Endoscopic Ultrasound - Fine Needle Aspiration (EUS-FNA) showed atypical cells with high grade dysplasia. Surgery was performed and histology showed pancreatic adenocarcinoma. Case 2 - 50year old male patient, hospitalized because of deep vein thrombosis and pulmonary embolism. The patient had cachexia, mild ascites, and presented chronic alcohol consumption. The patient had no abdominal pain, lipase was normal and malignancy was suspected. CT Scan raised the suspicion of a liver metastasis and a suspect pancreatic lesion. MRI showed no evidence of a liver or solid pancreatic mass, only a pancreatic cyst. Diagnostic paracentesis did not detect any tumor cells. EUS showed a $2.2 \times 1.8 \mathrm{~cm}$ cystic lesion in pancreas with an $8 \mathrm{~mm}$ solid content. Fluid analysis obtained was typical for a pseudocyst. Paracentesis was performed once more and showed increased lipase levels. Secretin MRI was performed for suspicion of pancreatic duct fistula. This was confirmed and successfully treated by ERCP. Case 3 - 56-year old female patient with known autoimmune thyroiditis hospitalized because of abdominal pain, weight loss and slightly increased lipase values. MRI raised the suspicion of a pancreatic mass and enlarged lymph nodes. EUS suspected an autoimmune pancreatitis. Fibrotic changes with lymphocyte infiltrate were presented in the histology obtained through EUS-FNA (IgC4 was not elevated). Therapy with steroids was started and after 3 months, MRI was normal and the patient was free of symptoms. CONCLUSION: EUS is a useful technique in clinical practice to establish a correct diagnosis in cases where radiology is inconclusive.

Key words: eus, mri, ct, diagnosis, radiology.

\section{UNCOMMON SITE FOR ENDOMETRIOSIS: THE CANAL OF NUCK}

Ioana-Daniela I Moise', Rayssa W Mahjoub', Mihaela-Ioana M Pitic ${ }^{1}$ Anca-Maria Manta MD, PhD²

"Carol Davila" University of Medicine and Pharmacy, Bucharest, Romania. 2Euroclinic Hospital, Bucharest, Romania.

BACKGROUND: Endometriosis is a benign polymorphic and multifocal disease, characterized by the presence of endometrial tissue outside the uterine cavity, associated with fibrosis and inflammatory reaction. The endometriotic implants are especially located in the pelvic cavity, but can also be found in atypical sites. Unusually, the endometrial tissue can be situated in the Canal of Nuck $(0,3-0,6 \%)$. This uncommon area is an embryonal rest of the parietal peritoneum that follows the round ligament of uterus and makes the link between the peritoneal cavity, the inguinal canal and the labia major. CASE PRESENTATION: We present a case of a 28 - years-old female, nulliparous, with no surgical history, who complained about dysmenorrhea (VAS 9), dyspareunia and a right inguinal nodule, which was associated with cyclical menstruation pain in this area. These symptoms have appeared one year ago. Imaging examinations (ultrasound and pelvic MRI) confirmed the presence of the formation with nonspecific characters without revealing the presence of intraperitoneal implants. The tumor was completely excised and the extemporaneous histopathological examination revealed the presence of endometrial tissue located on the round ligament. The immunohistochemistry confirmed the diagnosis. Postoperative, the treatment with contraceptives was applied and the patient evolution was favorable. CONCLUSION: Inguinal endometriosis is a very rare entity. Although the symptoms can guide us in cases of women at the reproductive age, with normal menstrual cycle, the diagnosis of endometriosis can be difficult. Therefore, we need imaging investigations for a complete evaluation of both extrapelvic and intraperitoneal sites. Histopathological examination after surgical excision is mandatory. Treatment involves a multidisciplinary approach, surgical excision and medical contraceptive treatment.

Key words: the canal of nuck, endometriosis, multidisciplinary approach.

\section{VARIABLE CLINICAL FEATURES IN DIGEORGE SYNDROME: A} COMPARISON OF TWO CLINICAL CASES

Rodica-Iulia Ch. Iorga', Andreea Florentina D. Șerban', Magdalena Budișteanu²

I"Carol Davila" University of Medicine and Pharmacy, Bucharest, Romania. 2"Prof. dr. AlexandruObregia" Clinical Hospital of Psychiatry, Bucharest, Romania.

BACKGROUND: DiCeorge syndrome or 22911.2 deletion syndrome has classically been characterized by the triad of clinical features including congenital cardiac defects, immune deficiencies, secondary to aplasia or hypoplasia of the thymus, and hypocalcemia due to small or absent parathyroid glands. However, it is now known to have a heterogeneous presentation that includes multiple additional congenital anomalies and later-onset conditions. CASE PRESENTATION: Patient A, a 16-year-old boy, was admitted in the Department of Pediatric Neurology for learning difficulties and socialization problems. He had a history of delayed speech development, growth failure during the first year of life and recurrent acute respiratory tract infections. The clinical evaluation showed a facial dysmorphic features (narrow forehead, synophrys, upslanting palpebral fissures, broad nasal root, short philtrum, dental malocclusion and micrognathia), long fingers and toes. The neurological examination revealed mild gait problems, mild muscular hypertonia; bradylalia with dyslalia. The psychiatric evaluation revealed bradipsyhia, learning difficulties, anxiety and antisocial behavior. Psychological evaluation indicated mild mental retardation (I.Q. 55). Right bundle branch block and sinus tachycardia were noticed on the electrocardiogram. Patient B, a 13-year-old boy, was referred to the department of Neurogenetics for particular facial features and toraciclumbar scoliosis. He had a history of frequent respiratory infections and chronic constipation; he also had a speech delay. Physical examination showed facial dysmorphism (malformed low-set ears, hypertelorism, upslanting palpebral fissures, synophrys, enlarged nasal root, prominent nasal pyramid, prominent maxillary, and micrognathia); long fingers and toes; thoracic-lumbar scoliosis; mild speech delay, concentration and attention difficulties. The laboratory investigations indicated hypocalcemia. In both cases genetic tests (array CGH, FISH) were performed; a 22911.2 deletion was identified in both cases. CONCLUSION: DiCeorge syndrome may present with a wide spectrum of clinical findings. These two patients had some common trades including dysmorphic facies with individual characteristics, behavioral problems and medical history of frequent respiratory infections. Hypocalcemia and cardiac problems are other important features of this syndrome. In conclusions, genetic tests for 22911.2 deletion should be taken into consideration in children with speech delay or learning difficulties or behavior problems, especially in association with dysmorphic features, heart disease and hypocalcemia.

Key words: DiGeorge syndrome, dysmorphic facies, behavior problems, developmental delay.

47. WHEN OLD DISEASES STRIKE BACK - A FASCINATING LEMIERRE'S SYNDROME CASE

Matei R. Iurea', Bianca D. Mihart'ํ, Octavian V. Baiu' ', Cristina Mări MD, PhD², Luminița Matroș' 
I"Iuliu Hațieganu" University of Medicine and Pharmacy, Cluj-Napoca, Romania. ${ }^{2}$ Emergency Pediatric Hospital, Cluj Napoca, Romania.

BACKGROUND: Lemierre's syndrome is caused by an anaerobe Gram-negative bacteria, Fusobacterium Necrophorum. Although it is a typical component of the bacteria from the oral flora, it can lead to pharyngitis, peritonsillar abscesses and even to more severe complications such as septic thrombophlebitis of the internal jugular vein, meningitis or even to the death of the patients. CASE PRESENTATION: This case report involves a 20 years old male patient who presented in the Emergency Department in an altered state following 7 days of antibiotic treatment for an upper respiratory infection. The patient presented with low blood pressure (80/59 $\mathrm{mmHg}$ ) fever, swollen lymph nodes and a tender neck, but also accused loss of appetite, muscle weakness and shortness of breath. The primary $x$ rays showed right lower lobe pneumonia so the patient was placed on an empiric treatment consisting of levofloxacin, ceftriaxone and penicillin $\mathrm{G}$, followed by supplemental oxygen and additional IV liquids. The second round of $x$-rays showed worsening infiltrate in the lungs with developing small layering effusion. The neck was still tender, therefore an ENT doctor was called for a consult, which later recommended a neck and chest computerized tomography. The CT showed thrombophlebitis and stenosis of the internal jugular vein, which correlated with the clinical symptoms is consistent with a Lemierre's Syndrome diagnosis. Therefore, the treatment was switched to piperacillin, clindamycin and metronidazole. Upon becoming stable, the patient was transferred to the surgical department to remove the clot from his internal jugular vein and later on performed a full recovery. CONCLUSION: Lemierre's disease should be considered as a possible diagnosis if the symptoms do not yield to antibiotics after 3-5 days. Although the disease is rare, it can still be lethal, reason why a fast and precise diagnosis is necessary, followed by a treatment consisting of antibiotics and possibly anticoagulants.

Key words: Lemierre's syndrome, Fusobacterium Necrophorum, stenosis of the internal jugular vein.

48.

\section{5-HTTLPR Polymorphisms Distribution Among 1497 Healthy Romanian Volunteers}

Tudor N. Hîrlea', Mădălina F.C. Lazăr ${ }^{1}$, Ioana Bunea PhD Stud. ${ }^{2}$, Lect. Adina Chiș', Romana Vulturar', Andrei Miu²

'I"Iuliu Hațieganu" University of Medicine and Pharmacy, Cluj-Napoca, Romania. ${ }^{2}$ Cognitive Neuroscience Laboratory, Babeș-Bolyai University ClujNapoca, Romania.

BACKGROUND: The serotonin transporter (5-HTT or SERT) is an integral membrane protein that plays a key role in regulating serotonergic synaptic function. 5-HTT is responsible for the reuptake of the serotonin from the synaptic cleft back into presynaptic neurons. It controls the duration, availability and signaling capacity of serotonin in the synapse and has been associated with anxiety related personality traits, depression related disorders and with reduced response to psychotherapy. The promoter of the 5-HTT gene contains a region called the serotonin-transporter-linked polymorphic region (5-HTTLPR), which modulates the transcriptional activity of the gene. This is a highly variable region due to an insertion/deletion polymorphism and a single nucleotide polymorphism (SNP) which determine the length and structure of the alleles. The 5-HTTLPR polymorphisms are of great relevance due to their involvement in the pathogenesis of multiple disorders, such as depression. MATERIALS AND METHODS: Our study assessed the frequencies of 5-HTTLPR genotypes in a group of 1497 physically and psychologically healthy Romanian volunteers, using the PCR- RFLP method. The bi-allelic distribution studies only the insertion/deletion polymorphism, differentiating between the short (S) and long (L) alleles. The tri-allelic distribution also includes a SNP (rS25531), resulting in alleles associated with a lower transcriptional activity $\left(\mathrm{S}^{\prime}\right)$ and others with a higher activity $\left(L^{\prime}\right)$. RESULTS: The bi-allelic distribution of 5 HTTLPR polymorphisms was: $484 \mathrm{~L} / \mathrm{L}(32 \%), 722 \mathrm{~L} / \mathrm{S}(48 \%)$ and 291 for S/S $(20 \%)$, respecting the Hardy-Weinberg equilibrium $\left(\chi_{2}=0.54\right)$. The alleles frequencies are: $56 \%(\mathrm{~L})$ and $44 \%(\mathrm{~S})$. The tri-allelic distribution was: 410 for $L^{\prime} / L^{\prime}$ genotype $(27 \%), 735$ for $L^{\prime} / S^{\prime}(49 \%)$ and 352 for $S^{\prime} / S^{\prime}(24 \%)$. These genotypes also respect the Hardy-Weinberg equilibrium $\left(\chi^{2}=0.41\right.$, with $L^{\prime}$ frequency $52 \%$ and $S^{\prime}$ frequency $48 \%$ ). CONCLUSION: Our study revealed an allele distribution consistent with previous findings on Caucasian populations. The major drawback of our study is that candidate gene studies are inconsistent with modern genetics findings, such as genome wide association studies (CWAS), as psychiatric disorders are now considered to be polygenic. ACKNOWLEDGEMENTS: This work was funded through grant number PNIII-P4 - ID - PCE - 2016-0840, the Romanian National Authority for Scientific Research, CNCS - UEFISCDI.

Key words: serotonin transporter, 5-HTTLPR, rS25531, Romanian healthy volunteers.

49. ASSESSMENT OF A DRUG-DELIVERY SYSTEM BASED ON SUPERPARAMAGNETIC NANOPARTICLES FOR BREAST CANCER THERAPY Andrei V. Alexandru', Sevinci Pop MD, PhD²

1"Carol Davila" University of Medicine and Pharmacy, Bucharest, Romania. 2"Victor Babeș" National Institute of Research and Development in Pathology and Biomedical Sciences, Bucharest, Romania.

BACKGROUND: Breast cancer is the most common cancer diagnosed among women, affecting 2.1 million women each year. A major problem in cancer therapy is the lack of specificity of chemotherapeutic drugs against tumors. In order to achieve efficient therapy, large doses of drugs were used for patient treatment which causes serious side effects. Therefore, current efforts in the biomedical field are focusing on developing strategies for targeted drug delivery. The aim of our work was to evaluate a nanosystem formed by superparamagnetic nanoparticles loaded with an antracyclinic antibiotic-violamycin B1. MATERIALS AND METHODS: MCF7 (adenocarcinoma) and $\mathrm{MCF} 12 \mathrm{~A}$ (non-tumorigenic mammary gland) cell lines were treated with unloaded nanoparticles (NP), VB1-loaded nanoparticles (VB1-NP) and VB1 solution at different concentrations: $0.025 ; 0.05$ and $0.1 \mathrm{mg} / \mathrm{ml}$ for $24-48 \mathrm{hrs}$. The cellular viability was assessed by MTS method, which measures the quantity of reduced tetrazolium products, as an indicator of cellular metabolism. The cytotoxicity of nanosystems was tested by measuring the Lactate dehydrogenase (LDH) release into cell culture media. The anti-tumor activity of NP-VB1 was proved by real-time monitoring of treated cells on xCELLigence platform and by RealTime-Clo ${ }^{\mathrm{TM}}$ Annexin $\mathrm{V}$ Apoptosis Assay. The intracellular accumulation of NPS in lysosomes of cells was quantitatively assessed by fluorescence microscopy using LysoSensor Green DND 189 staining and by videomicroscopy. RESULTS: Both cell lines treated with $0.025 \mathrm{mg} / \mathrm{ml}$ of NPs showed cellular viability and cytotoxicity values similar to control experiments, untreated cells. For $0.05 \mathrm{mg} / \mathrm{ml}$ and $0.1 \mathrm{mg} / \mathrm{ml}$ there was a significant decrease in cell viability induced by the cytotoxicity of the NPs at these concentrations. The VB1-NP treatment of cells significantly decreased their proliferation rate and finally induced cellular apoptosis. The anti-tumor activity of VB1 was maintained by nanoparticles delivery system, but the apoptosis process is delayed. This could be explained by the nanoparticles endocytosis process in cells, and respectively by VB1 releasing time from NPS. CONCLUSION: This study demonstrated the efficacy of a superparamagnetic nanoparticles - VB1 drug-delivery system on breast cancer cells. ACKNOWLEDGEMENTS: This work was supported partially by the Ministry of Research and Innovation in Romania, under Program 1 - The Improvement of the National System of Research and Development, Subprogram 1.2 - Institutional Excellence - Projects of Excellence Funding in RDI, Contract No. 7PFE/16.10.2018 and by Core Program PN 19.29.01.04

Key words: drug-delivery system, superparamagnetic nanoparticles, breast cancer therapy, violamycin B1.

\section{DECORIN AS A POSSIBLE MEDIATOR OF AMYLOID BETA PATHOLOGY IN ALZHEIMER'S DISEASE}

Robert F. Haret'Per Nilsson ${ }^{2}$

I"Carol Davila" University of Medicine and Pharmacy, Bucharest, Romania. ${ }^{2}$ Division of Neurogeriatrics, Department of Neurobiology, Care Sciences and Society, Center for Alzheimer Research, Karolinska Institutet, Solna, Sweden.

BACKGROUND: Alzheimer's disease (AD) is the most common neurodegenerative disease and the major form of dementia in the elderly. It is characterized by aberrant protein aggregation, notably amyloid beta $(A \beta)$ peptide accumulation and the formation of intracellular neurofibrillary tangles from hyperphosphorylated tau. Protein aggregates in the cell are cleared by autophagy, a mechanism which has been shown to be impaired in $\mathrm{AD}$. Decorin is a small leucine-rich proteoglycan of the extracellular matrix which has been proven to induce autophagy in endothelial cells and, through mass spectrometry, has also been found in abnormal concentrations in the cerebrospinal fluid of AD mouse models. MATERIALS AND METHODS: We investigated the role of decorin and autophagy in $A B$ pathology in vivo using amyloid precursor protein (APP) NL-F and APP NL-G$F$ knock-in mouse models. Both APP NL-F and APP NL-G-F models avoid potential artifacts introduced by APP overexpression by using a knock-in 
approach to express APP at wild-type levels while producing elevated pathogenic $A \beta$. Sections $4 \mu \mathrm{m}$ (paraffin embedded) thick were immunostained for decorin, $A \beta$ and different neuronal markers. Quantification was performed with Fiji (ImageJ) imaging software. RESULTS: Both APP NL-F and APP NL-G-F knock-in 12 months old mice expressed a significant $A \beta$ pathology compared to wild type (WT). Decorin was present in both the cortex and the hippocampus in the brains of WT and AD mouse models. We then identified that the majority of neurons in the cortex that expressed decorin were a subpopulation of the parvalbumin (PV) positive interneurons. In the hippocampus, decorin was, surprisingly, colocalized with pyramidal neurons only from CA2 and with PV expressing interneurons in the regions CA1, CA3 and DG. CONCLUSION: As far as we know, it is the first time decorin has been studied in the context of the central nervous system. Given that autophagy has been shown to mediate the secretion of pathological $A B$ in the extracellular space and taking into consideration the strong relationship between decorin and autophagy, this proteoglycan shows great promise in further explaining the complete pathology of $A B$. Decorin may even constitute the basis for discovering a new biomarker fo $A D$, however, further research is necessary.

Key words: Alzheimer's Disease, amyloid beta, decorin, autophagy, parvalbumin interneurons.

\section{EVALUATING HEALTHY CELLS VIABILITY EXPOSED TO CANCEROUS CELL} DESTRUCTION DESIGNED MAGNETITE NANOPARTICLES

Gabriel Cristian E. Vacaru', Luchian I.A. Marinescu', MihaelaAndreea C. Podeanu', Alexandra C.F. Vaduva', Roacho-Pérez Jorge Alberto $^{2}$, Domínguez Celia Nohemí ${ }^{2}$

'University of Medicine and Pharmacy of Craiova, Craiova, Romania. 2Universidad Autonoma de Nuevo Leon, Facultad de Medicina, Monterrey, Mexico.

BACKGROUND: Over the last decade, great progress has been done in developing nanoparticles (NPS) that might be used for oncology disease detecting and treatment. Also, NPs can be engineered for contrast imagining use, targeted drug delivery and even as therapeutical agents to initiate tumour cell death. Thus, making us question about their biocompatibility within healthy cells. MATERIALS AND METHODS: Magnetite NPS were synthesized by chemical co- precipitation. We used two precursor solutions: $100 \mathrm{ml}$ of ferric chloride and $100 \mathrm{ml}$ of ferrous sulfate. NPs were coated with medium molecular weight chitosan previously dissolved in $90 \mathrm{ml}$ of a $2 \%$ glacial acetic acid solution. In order to measure the NPs capacity to cause cell death, an MTT assay was made.

A mesenchymal stem cells primary cell culture from mouse bone marrow was cultured, using DMEM medium. Cells were exposed by quadruplicate to the nanoparticles. The evaluated concentrations were logarithmic series from 5,000 to $5 \mathrm{ng} / \mathrm{ml}$. Cell death was measured after 24 and $48 \mathrm{~h}$ pos exposure. For MTT assay, the cells were incubated with an MTT solution fo 4 hours. After incubation, the medium was retired, and formazan was dissolved in $50 \mu \mathrm{l}$ of $\mathrm{pH}_{3}$ isopropanol. The absorbance was read at $490 \mathrm{~nm}$. The viability of non-exposed cells was set to $100 \%$ and samples viability calculated with a mathematical equation. RESULTS: All the samples viability turned out to be above $70 \%$ of cell viability marker. The American standard IS0-10993-5 specifies that all the biomedical materials must present their viability above $70 \%$ to be considered a biocompatible material. CONCLUSION There are no significant differences between control (non-exposed cells) and all the samples we analyzed. Bearing in mind, ISO-10993-5 regulations, that all the samples above $70 \%$ of viability are considered non-dangerous samples, future therapeutic prospective can be taken into account. Considering other MNPs toxicity related studies, we confirm that the MNPs dose used for drug delivery is safe for the unaffected tissues and it is not necessary to use extremely low frequency magnetic field in order not to negatively interfere with the healthy cells. Also, literature specifies that different cellular Redox environment distincts normal and cancerous cells in terms of MNPs action.

Key words: nanoparticles, magnetite, cancer, chitosan, mesenchymal stem cells.

52.

GENOME-WIDE ASSOCIATION STUDY OF BREAST CANCER IN ROMANIAN POPULATION

Sorina-Diana S. Cocolea', Paul Iordache MD, PhD²

"Carol Davila" University of Medicine and Pharmacy, Bucharest, Romania.

${ }^{2} \mathrm{School}$ of Science and Engineering, Reykjavik University, Reykjavik, Iceland.
BACKGROUND: Breast cancer remains a pathology with great social-cultural impact, being the first type of cancer ever described. Previous studies have found breast cancer to have the second highest incidence of all neoplasms worldwide and the highest mortality in the European female population. To identify the role of certain alleles in this pathology we used a genome wide association study (CWAS). MATERIALS AND METHODS: Patients included in the study were hospitalized in Surgery Ward III of the Bucharest Emergency Teaching Hospital. Patient inclusion criteria consisted of: the existence of primary cancer and not metastases; topographic localization; age between 18 and 80; confirmation by pathology exam; controls were selected using the following criteria: being in the same 5 -year age group as the patients; patients from the same clinic with a different pathology; self-declared or observed Rroma ethnicity. Steps taken to filter the data file using PLINK toolset: 1) Elimination of individuals with missing genotype data $10 \%$ (mind 0.1 ). 2) Elimination of SNPS with an allelic frequency-MAF $<5 \%$ (maf 0.05 )->Eliminates 11.974 variants->92.416 variants remain. 3) Elimination of SNPS with missing genotype data of $10 \%$ of individuals (geno 0.1)>Eliminates 74 variants->92.342 variants remain. 4) Exclusion of SNPS not in Hardy-Weinberg equilibrium (hwe 0.001)->Eliminates 445 variants->91.897 variants. 5) Association test generating plink.assoc file. Final results: 91.897 variants with 278 Patients and 1378 Controls. RESULTS: Our study identified 26 significant (10-5) SNPs linked with breast cancer, out of which one was previously reported. The marker $\mathrm{rs}_{1219648}$ had the highest level of significance. It's located in the FGFR2 gene that is involved in a wide array of cancers through activation of the RAS-MAPK and PI3K-AKT pathways. We also identified 5 novel SNPS previously unreported with possible biological implications. The main limitation of the study was the small number of patients, but nevertheless it remains an important first step in CWAS analysis of the Romanian population. CONCLUSION: In conclusion, most SNPS previously validated don't seem to be present or have a statistically significant association in a Romanian population and future studies would be necessary for confirmation. These studies could also include the new SNPS described in this study.

Key words: CWAS, breast cancer, SNPS, BRCA.

53. IMMUNOHISTOCHEMICAL FEATURES OF CYTOTROPHOBKAST INVASION IN THE UTERINE-PLACENTAL AREA AND MYOMETRIUM IN IRON DEFICIENCY ANEMIA DURING PREGNANCY

Hennadii P. Petrochenkov', Viallanta 0 . Tiulienieva', Olena Tiulienieva ${ }^{1}$

'Bukovinian State Medical University, Chernivtsi, Ukraine.

BACKGROUND: During pregnancy, the key mechanism in the morphogenesis of the uterine-placental region is cytotrophoblastic invasion (CTI) - the process of migration of cytotrophoblast (CT) cells from the placenta to the endometrium and myometrium to establish uterine-placental circulation, which determines the possibility of compensatory-adaptive reactions in the uterine-placental complex and favorable termination of pregnancy. Iron deficiency anemia in pregnant women (IDAP) is one of the most acute problems of modern obstetrics. MATERIALS AND METHODS: 60 biopsies obtained in the Caesareo section, including 28 of physiological pregnancy, 32 - IDA of pregnant. Term of gestation was 37-40 weeks. The following techniques were used:1) immunohistochemical studies with primary antibodies against the interval of metal 2 and 9, placental lactogen with the thermal exposition of the antigen(Dako); 2) histochemical method of determining fibrin and collagen fibers according to N.Slinncheko; 3 ) coloring of hematoxylin and eosine. RESULTS: The study showed the spread of CT not only in the area of the spiral arteries of the endometrium, but also the incorporation of EC into the walls of the myometrial segments of arteries. Immunohistochemical determination of CT invasiveness: during physiological pregnancy, the activity of metalloproteinases 2 is $0.232 \pm 0.0012$, metalloproteinases 9 are $0.219 \pm 0.0014$, with IDA of pregnant the activity of metalloproteinases 2 - in the range of $0.238 \pm 0.0015$ $0.229 \pm 0.0014$, metalloproteinases $9-0.225 \pm 0.0014^{-} \quad 0.187 \pm 0.0014$ Immunovisualization for placental lactogen during physiological pregnancy was: IC $-0.314 \pm 0.0022$, EC $-0.109 \pm 0.0022$; in the observations at the time I passed: IC in the range of $0.326 \pm 0.0024-0.256 \pm 0.0021, E C 0.108 \pm 0.0022$ $0.074 \pm 0.0024$. At the same time, the gestational transformation of the walls of the arteries of the UPA was not complete. CONCLUSION: There is a deepening of cytotrophoblast invasion into the structures of the uteroplacental region and an increase in the lining distance of the endothelium-bearing cytotrophoblast of the artery walls and, as a result, a large dilatation of the lumen of the spiral and radial uterine arteries to 
improve gas exchange in the mother-placenta system. However, the invasive ability and synthetic activity of the cytotrophoblast and, as a consequence, the adequacy of gestational transformation of the structures of the placental uterine bed depend on the stadium of IDA of pregnant women.

Key words: uterine-placental area, cytotrophoblastic invasion, iron deficiency anemia.

54. POTENTIAL THERAPEUTIC EFFECT OF THYMOQUINONE ON NEUROBLASTOMA USING TWO-DIMENSIONAL AND THREE- DIMENSIONAL CULTURE MODELS

Andrei A. Tanasov', Katarina Momir Dunjic², Jan J.S. Stanič3 ${ }^{3}$, Minea Šućur ${ }^{4}$, Wassim Abou Kheir ${ }^{5}$

1"Carol Davila" University of Medicine and Pharmacy, Bucharest, Romania. 'University of Belgrade, Faculty of Medicine. 3University of Ljubljana, Faculty of Medicine. ${ }^{4}$ University of Maribor Faculty of Medicine. ${ }^{5}$ American University of Beirut, Lebanon.

BACKGROUND: Thymoquinone (TQ) is a bioactive component derived from the black seeds of the medicinal plant Nigella Sativa, with proven cytotoxic effects in several cancer cell lines, such as neuroblastoma. The study tests the antitumoral properties of Thymoquinone on neuroblastoma cells in a two-dimensional assay, the Cell Viability Assay using Trypan Blue, and a three-dimensional one, the Sphere Formation Assay. MATERIALS AND METHODS: In the first assay, IMR32 neuroblastoma cells were seeded in three 16-well plates, incubated and treated with TQ of increasing concentrations. At each timepoint, cells were collected, resuspended, treated with Trypan Blue and the viable neuroblastoma cells were counted using a hemocytometer. The second assay used a complex threedimensional culture model, obtained by suspending the IMR32 cells in Matrigel. The solution was placed gently around the rim of individual wells of a 24-well plate and incubated for solidifying. Media with TQ of increasing concentrations was added in the center of the wells and changed every 2 days. After 7 days, spheres were counted, measured using Zeiss Microscopy ZEN software and the sphere-forming unit was calculated for each drug concentration. RESULTS: In the Trypan Blue assay, $10 \mu \mathrm{M}$ TQ decreased the proliferative activity of IMR32 by $50 \%$ at 24 hours, by $20.58 \%$ at 48 hours and by $72.22 \%$ at 72 hours, while the $25 \mu \mathrm{M}$ TQ had a $94.21 \%$ inhibitory effect at 48 hours and completely destroyed all cells at 24 and 72 hours. The increasing concentrations of TQ also had inhibitory effects on cancer stem cells spheres' both sizes (53.279\% average size decrease) and sphere forming units ( 3.5 for control, 2.65 for $0.1 \mu \mathrm{M} \mathrm{TQ}, 1.85$ for $1 \mu \mathrm{M}$ TQ and 0.75 for $3 \mu \mathrm{M}$ TQ). CONCLUSION: The study shows that TQ significantly affects the proliferation and survival of cancer cells of brain origin. The existence of cancer stem cells population within this type of tumor has been correlated with poorer prognosis; this is why it is crucial to use drugs that target this small population for preventing recurrence. TQ showed great dosedependent inhibition of size and number of sphere-forming units, revealing its effective anti-cancerous properties.

Key words: neuroblastoma, cancer stem cells, thymoquinone, sphereforming unit

55. PRF-PHYSICAL CHARACTERIZATION AND POSSIBLE CLOT AUGMENTATION Raluca C.S. Bocai', Nicoleta N. Asavei', Rustin C. Maria Madalina', Bogdan M. Matei', Valentin Popescu', Bogdan Mastalier', Mihaelá G. Moisescu', Traian Pătrașcu²

"Carol Davila" University of Medicine and Pharmacy, Bucharest, Romania. 2"Dr. I. Cantacuzino" Clinical Hospital, Bucharest, Romania.

BACKGROUND: The increasing demand for regenerative therapies and biomaterials that can potentially restore diseased and injured tissues has lead to intensive research on PRF and associated subgroups. Platelet-rich fibrin (PRF) is a natural fibrin-based biomaterial used in wound healing and bone regeneration. It contains cytokines and growth factors that increase vascularization and guide epithelial cell migration to its surface. It is estimated that the prevalence of chronic wounds in developed countries is around $1 \%$ to $2 \%$ of the population and these numbers will rise as the ageing population increases. Chronic wounds seem to be a significant problem for the health system and also for the quality of life of patients and their families. In consequence, the aim of this study was to analyse the possibility of obtaining a bigger PRF clot from the same amount of blood in order to cover larger defects of chronic wounds. MATERIALS AND METHODS:
For this reason, we used different substances for PRF augmentation like vitamin $C$ or calcium. By augmentation we mean the qualitative or quantitative improvement measured by different criteria like volume, colour or the degree of hydration. We hypothesized that vitamin $\mathrm{C}$ and calcium could promote wound healing by enhancing the biophysical properties of the PRF clots. It is known that vitamin C is a cofactor for collagen synthesis and a primary antioxidant and calcium is a key factor in the coagulation cascade. Therefore, we examined the influence on the weight, volume, hydration, colour and other biophysical properties when augmenting PRF clots with these substances in different doses compared with the standard protocol. RESULTS: As a result of the measurements made, we observed an increase in volume and a better hydration compared to the mean values of the standard ones, especially in the calcium group. The results can have future applications and potential for more effective PRF treatments at very low costs. CONCLUSION: In conclusion, we obtained very promising results compared to the standard ones, in the hope of finding a safer, more efficient and feasible treatment for these patients.

Key words: PRF, wound healing, characterization, biomaterial, calcium, vitamin c.

\section{THE BENEFICIAL ROLE OF BACILLUS SPORES ON ACETAMINOPHEN ACUTE} LIVER INJURY

Andrei C.I. Mitre' ${ }^{1}$ Adrian Catinean' ${ }^{1}$ Maria-Adriana Neag' ${ }^{1}$ Anca-Dana Buzoianu

"Iuliu Hațieganu" University of Medicine and Pharmacy, Cluj-Napoca, Romania.

BACKGROUND: The liver is the main organ involved in maintaining the body's homeostasis. Acetaminophen ( $\mathrm{N}$-acetyl-p-aminophenol or paracetamol), an over-the- counter drug, is one of the most used analgesic and antipyretic agent in the world. It is known that acetaminophen (APAP) in high doses may lead to acute liver failure. Intoxication with APAP is the main cause of acute liver toxicity in both the US and Europe. Probiotics have been shown to have beneficial effects in several ailments from gastro- intestinal disorders (inflammatory bowel diseases, liver diseases) to allergy, metabolic disorders or cancer. This study was performed to evaluate the possible protective effect of Bacillus spp. spores on acute hepatic injury induced by APAP overdose in rats. MATERIALS AND METHODS: We used Wistar white male rats. A total of 35 rats were randomly divided into seven groups $(n=5)$ : group I served as control and received only the vehicle, Carboxymethylcellulose (CMC); group II received Silymarin (SIL) (100mg/kg/day); group III received MegaSporeBiotic (MSB) (1X109CFU/day); group IV received APAP $(2 \mathrm{~g} / \mathrm{kg})$ - this group served as the model of hepatotoxicity; group $\mathrm{V}$ received APAP $(2 \mathrm{~g} / \mathrm{kg})$ and SIL $(100 \mathrm{mg} / \mathrm{kg} / \mathrm{day})$; group VI received APAP (2g/kg) and MSB (1X109 CFU /day); group VII received APAP $(2 \mathrm{~g} / \mathrm{kg})$, SIL (100mg/kg/day) and MSB (1x109CFU/day). Liver for histopathological examination and blood samples were collected on the last day of the experiment ( 48 hours after the Acetaminophen dose). We measured hepatic injury markers CPT and COT, total antioxidant capacity (TAC) and zonula occludens (Z0), Tumor Necrosis Factor (TNF) $\alpha$ and interleukin (IL) 6. RESULTS: Histopathological, pre-treatment with SIL, MSB or SIL+MSB significantly relieves changes in hepatic parenchyma as compared to changes in group with APAP alone. COT, GPT, ZO, TAC and IL- 6 values were significantly improved for all treated groups compared to APAP. TNF- $\alpha$ was lower compared to APAP only in the SIL+MSB group (g. VII). CONCLUSION: Our study revealed that the supplement based on Bacillus spp. spores has a protective effect on acute hepatic injury induced by APAP.

Key words: acetaminophen, acute liver injury, Bacillus spores.

\section{THE IMPORTANCE OF PSYCHOLOGICAL FACTORS IN PATIENTS WITH CARDIOVASCULAR PROFILE}

Ivanes Igor Igor', Cernițanu Mariana'

"Nicolae Testemițanu” State University of Medicine and Pharmacy, Chișinau, Moldova.

BACKGROUND: Identification and analysis of the psychological factors that contribute to the occurrence and maintenance of cardiovascular pathology. Friedman and Rosenman (1959) describe the type A of personality which is predisposed for cardiovascular disease. MATERIALS AND METHODS: In study have participated 300 respondents over 45 years old with cardiovascular diseases including - $165(55 \%)$ were with hypertension, $76(25.3 \%)$ with ischemic heart disease, $38(12.7 \%)$ with cardiac arrhythmias and $21(7 \%)$ 
with other cardiac pathologies. The questionnaire included 18 items grouped in: - socio- demographic data; - questions aimed at highlighting the psychological factors with potential impact on the health status of the respondents; RESULTS: According to the obtained results, the majority of the respondents $61.7 \%$ were married. However, the rate of divorced people and widows was quite high at $20.3 \%$ and $18 \%$ respectively. These data allowed maintaining the idea that loneliness is a psychological problem that generates a long term stressful situation and directly influence the cardiovascular system activity. To the question regarding the consequences created by the negative, stressful memories on the state of health: 145 $(48.3 \%)$ respondents answered that in these situations they have exacerbations of the clinical symptoms (blood pressure increases, precordial pain, palpitations, general weakness), 52 (17.3\%) respondents need to consult the family doctor and / or the specialist doctor (cardiologist), $110(36.7 \%)$ respondents answered that they need to increase the dose of drugs and only $3(1 \%)$ respondents have no change in their somatic state or behavior. CONCLUSION: Personality type A is predisposed to cardiovascular disease, being characterized by ambition, perseverance and increased combativity, the feeling of urgency and that of competition. $70 \%$ respondents regret a dream, wishes or aspirations unrealized, $47.3 \%$ respondents consider themselves sensitive people, easy to offend, $70.7 \%$ respondents said they rarely abstain from crying, $75.3 \%$ respondents think about the negative events of the past, $81 \%$ respondents think they have some reservations regarding the degree of achievement of the major goals in life until the questioning stage. Some prophylaxis measures would be to maintain the emotional-affective balance, a comfortable environment of peace and equilibrate relationship with others. Person should be encouraged to process his states of excessive anxiety, irritability, and aggression trough special psychological technics as meditation, mindfulness and biofeedback.

Key words: psychological, predisposing, factors, patients with cardiovascular profile.

\section{ASSESSMENT OF THE RISK OF EXACERBATION IN CHRONIC OBSTRUCTIVE} PULMONARY DISEASE PATIENTS

Alexandra Andrei Plahotniuc', Serghei Covantev' ${ }^{1}$, Olga Corlateanu', Alexandru Corlateanu

'Nicolae Testemițanu"State University of Medicine and Pharmacy, Chișinau, Moldova.

BACKGROUND: The number of exacerbations presents a significant component of indices that predict the COPD (chronic obstructive pulmonary disease) severity and also can serve as an independent prognostic factor for the COPD-related quality of life, deterioration and mortality. The prediction of COPD exacerbation still represents a challenging task fo clinicians. MATERIALS AND METHODS: In the study there were included 433 patients. Spirometric data (FEV1 (forced expiratory volume in 1 second), FVC (forced vital capacity), FEV1/FVC) and e-BODE (exacerbation, body-mass index, airflow obstruction, dyspnoea and exercise) were analyzed in aspect of different COPD classifications: COLD (The Global Initiative for Chronic Obstructive Lung Disease) 2001, GOLD ABCD 2011, GOLD ABCD 2017 and phenotypes. RESULTS: 433 cohort participants were represented by 352 $(81 \%)$ men and $81(19 \%)$ women with mean age $62,7 \pm 9,8$ years. All GOLD classifications tended to underestimate the risk of exacerbation (COLD 200 AUC (area under the curve) - 0.623, GOLD ABCD AUC - 0.546 and COLD ABCD 2017 AUC - 0.545) whilst e-BODE index and phenotypic classification demonstrated superiority in risk prognosis (e-BODE AUC 0.908 and phenotypic classification AUC 0.995). CONCLUSION: In this study E-BODE index and phenotypic classification has demonstrated the best discriminatory performance in assessment of the risk of COPD exacerbation prognosis in comparison with GOLD categories.

Key words: chronic obstructive pulmonary disease, exacerbation, e-BODE, phenotype, GOLD.

59. CHANGES OF THE BILE HOMEOSTASIS IN PATIENTS WITH CHRONIC ACALCULOUS CHOLECYSTIC COMORBID WITH BRONCHIAL ASTHMA

Zahar Euvheniovich Marfiuk', Tetana Dudka MD, PhD'

'Bukovinian State Medical University, Chernivtsi, Ukraine.

BACKGROUND: Chemical bile content, spectrum of microbial and parasitic antigens in patients with $B A$ and CAC depending on the degree of severity of BA. MATERIALS AND METHODS: The study was conducted on 92 patients: 30 patients with BA of mild and moderate severity of a persisting course (the 1st group), 30 patients with BA of mild and moderate severity of a persisting course with comorbid CAC in exacerbation stage (the 2nd group), 32 patients with CAC in exacerbation stage (the 3 rd group), the control group included 30 practically healthy individuals of an appropriate age. RESULTS: Depending on available comorbidity the biochemical analysis of bile (Bportion) in patients with CAC showed a number of changes. For example, the content of total lipids in patients of the 2 nd and 3 rd groups was 1,8 and $2,5$ times higher than those of healthy individuals ( $p<0,05)$, while among patients of the 1st group it only had a tendency to increasing ( $>0,05)$. Cholesterol content in bile in the 2 nd and 3 rd groups of comparison elevated those of practically healthy people by 1,3 and 2,1 times $(p<0,05)$, and in patients of the 1 st group it was within the normal limits ( $p>0,05)$. At the same time, in the course of investigation a reliable increase of bilirubin level in bile was found in all groups - $26,8 \%, 43,5 \%$ and 15,1 \%as much $(p<0,05)$ as compared to practically healthy people. CONCLUSION: The results of the study demonstrate increased content of indirect bilirubin fraction in the blood of patients with BA is associated with increasing coefficient of bile pleiochromia and increased lithogenicity of bile that can be considered risk factors promoting formation of pigment calculi. Since in patients with isolated course of CAC bile is mostly saturated with cholesterol and there is a risk of cholesterol calculi formation, then in patients with BA there is a high probability of formation of mainly mixed or pigment calculi.

Key words: chronic acalculous cholecystitis, brocnhial asthma, bile.

\section{CLINICAL AND INSTRUMENTAL MARKERS OF ACUTE MYOCARDIAL INFARCTION COMPLICATED WITH ACUTE HEART FAILURE FLOW EVALUATION}

Anastasiia V. Hoian', Tetiana Ilashchuk

'Bukovinian State Medical University, Chernivtsi, Ukraine.

BACKGROUND: Determination of prognosis within first year after acute myocardial infarction (AMI) remains one of the most actual problems of cardiology. MATERIALS AND METHODS: 368 patients were examined. Transmural AMI occurred in $141(38.32 \%)$, macrofocal - in $166(45.11 \%)$ and microfocal - in $61(16,57 \%)$ cases. $123(33,42 \%)$ died throughout observation period, in particular, $94(25,54 \%)$ patients - during 28 -day staying in hospital, and $29(7,88 \%)$ - during a year of observation. All patients were divided into 2 groups: group 1 - with favorable, and group 2 - with fatal AMI outcome RESULTS: Patients who died were averagely 9 years older comparing those with favorable outcome. Males were prevalent amongst $(p<0,001)$. Repeated AMI was registered more frequently in 2 group $(p<0,001)$. Class $2-4$ ALVF signs by Killip were significantly more frequent in group 2 ( $p<0,001)$. Singlefactor regression analysis results pointed on fact that risk of lethal event occurrence increased with age: increase of risk by a factor of 1.5 follows each additional 5 years over 50 . Risk of lethal event appearance raised twice with every ALVF class by Killip increase, 1.3 times more in patients with DM, 1.15 times more in case of obesity presence, three times more in patients with chronic heart failure, 1.2 times more in case of ejection fraction below $40 \%$ detection during 1-2 days after patient's admission, and 4.5 times more in case of anterior AMI localization. IL- $1 \alpha$ content analysis revealed it's significant predominance in group 2 patients $(48,94+7,05$ vs $22,43+3,41 \mathrm{pg} / \mathrm{ml}, \mathrm{p}<0,01)$. IL-6 level was markedly higher in group 2 patients as well $(51,63+7,86$ vs $16,84+3,94 \mathrm{pg} / \mathrm{ml}, \mathrm{p}<0,01)$, and level of antiinflammatory cytokine IL-10 was less in group $2(2,45+0,51$ vs $4,03+0,73 \mathrm{pg} / \mathrm{ml}, \mathrm{p}>0,05)$. Tumor-necrotizing factor(TNF) and neopterine(Np) levels analysis in groups indicates significant predominance of these both values in group $2: 63,41+3,78$ vs $43,1+2,62 \mathrm{pg} / \mathrm{ml}$ for TNF $(p<0,01)$ and $24,28+4,32$ vs $15,08+1,76 \mathrm{nmol} / /$ for $\mathrm{Np}(p<0,05)$. CONCLUSION: Elder age, higher class of ALVF, presence of DM and CHF, anterior localization of AMI are independent predictors of lethal event development in patients with AMI and ALVF. Increase in L-1 $\alpha$, IL-6, TNF and Np with worsening of EchoKC results favor increase of lethal event onset probability in mentioned category of patients.

Key words: acute myocardial infarction (AMI), acute left-ventricular failure (ALVF), prognosis, risk factors.

\section{COMPLEX THERAPY OF PATIENTS WITH BRONCHIAL ASTHMA VIA USINC} NUCLEINAT

Petro Tarasovich Varkhomii', Tetiana Dudka MD, PhD

'Bukovinian State Medical University, Chernivtsi, Ukraine. 
BACKGROUND: Nucleinat is a physiologically active compound of a natural origin that is a bioactive extract of yeast RNA which reveals into an immunotropic activity. The aim of the research is to study the effectiveness of the use of Nucleinat in the therapy of patients with bronchial asthma. MATERIALS AND METHODS: 50 patients with BA of average seriousness with persistent course in the period of exacerbation and 20 practically healthy people were examined. The patients of a test $\mathrm{K}$ group ( 25 people) were given base therapy. The main 0 group ( 25 people) were additionally given Nucleinat $500 \mathrm{mg} 3$ times on a day 30 days long. RESULTS: Since the therapy of 0 group patients includes Nucleinat - the dynamic indexes of intensity of lipid peroxidation were different from the initial ones in the dynamic treatment. Thus, malonic content in blood plasma after the therapy in 0 group has reduced in 1,6 times $(p<0,05)$ while the changes in $\mathrm{K}$ group were false $(p>0,05)$. Antioxidant properties of Nucleinat are caused by its ability to normalize the activity of antioxidant factors. The dynamic indexes of restored glutathione content in plasma credibly increases by $34,8 \%(p<0,05)$ while the indexes of $K$ group had only an upward trend. The result of this process was a significant credible reduce of NO excess and inflammatory and fibrogenic cytokines by inducible NO-synthase. In particular, NO content in plasma in 0 group has reduced by $24,9 \%(p<0,05)$, while in $K$ group patients the decrease was $7,9 \%(p<0,05)$. CONCLUSION: Complex therapy of patients with BA of average severity with persistent course in the period of exacerbation including the additional inclusion of Nucleinat encouraged reducing the intensity of lipid peroxidation, oxidative modification of proteins and nitrositive stress, restoration of antioxidant factors activity and natural system of detoxification.

Key word: bronchial asthma, Nucleinat, treatment.

62. DEPENDENCE OF PROLACTIN LEVEL ON PSYCHOSOMATIC STATE IN PATIENTS WITH STABLE ANGINA OF TENSION AND GASTROESOPHAGEAL REFLUX DISEASE

Olha Evgenevna Mandryk', Vitalii Smandych, Yana Torerika, Hryniuk Olha Yevhenivna MD PhD', Victoria Drozd MD PhD', Khukhlina Oksana Sviatoslavivna'

'Bukovinian State Medical University, Chernivtsi, Ukraine.

BACKGROUND: Depressive disorder and anxiety lead to the onset of ischemic heart disease (IHD), aggravate its clinical course and prognosis causing chronical stress. One of the main mediators and, correspondingly, indicators of both acute and chronic stress is the hormone of prolactin (PL). MATERIALS AND METHODS: In the study, there were included 88 patients with stable angina of tension I - II functional class with comorbid CERD (A, B, C) aged from 48 to 79 years. Serum PL level was determined in all patients under study by an immunoluminescent analysis method under minimal stress conditions. The upper limit of the PL norm level was regarded to be the average value between the upper limit of the norm for men and women which is $22,5 \mathrm{ng} / \mathrm{ml}$. All the women in the study group were in the postmenopausal period. To determine their psychosomatic condition the patients were to fill out the HADS (Hospital of Anxiety and Depression Scale) questionnaire, which includes 14 questions and consists of two subscales with 7 questions: HADS-D (depression) - to determine depression and HADSA (anxiety) - to determine anxiety. RESULTS: In-depth analysis of the study results revealed a direct high correlative connection $(r=0,91)$ both between the serum PL level and the number of points by the anxiety scale and those by the depression scale $(r=0,79)$ testifying for the dependence of PL level on the patient's psychosomatic condition. According to the ROC analysis data, specificity of PL level growth in patients with diagnosed anxiety by the HADS questionnaire was 98,4 and sensitivity 87,8. CONCLUSION: According to the HADS data, there has been revealed anxiety in patients under study, and the number of points by the scale describing it highly and directly correlates with the serum prolactin level of these patients. High specificity and sensitivity of the serum PL level growth in relation to the presence of anxiety by the HADS-A scale has been established.

Key words: Gastroesophageal reflux diseases, PL level.

63. GASTROESOPHAGEAL REFLUX DISEASE COMORBID WITH COLD: THE ROLE OF NITRO-OXIDATIVE STRESS IN PROGRESSION

Mykola Mykolayovych Shevchuk', Inna Dudka

'Bukovinian State Medical University, Chernivtsi, Ukraine.

BACKGROUND: Recently, emphasis was placed on the role of nitro-oxidative stress in the pathogenesis of COLD exacerbations, which is confirmed by the rising concentration of thionitrites, peroxynitrites and other NO metabolites in the exhaled air.

Aim: To determine the degree and the role of endothelial dysfunction (ED) in the onset and progression of GERD in patients with COLD. MATERIALS AND METHODS: 40 patients with COLD stage II (COLD 2B) have been examined, including 10 patients without accompanying pathology (Group 1), 10 with endoscopic negative (EN) GERD (Group 2), 10 with endoscopically positive non- erosive (EPN) GERD (Group 3), 10 - with endoscopically positive erosive (EPE) GERD (Group 4). RESULTS: In patients with combined COLD and GERD, there was an increase in the NO content in the blood (1.6-2.0 times higher) than in patients with isolated COLD. The activity of iNOS in patients within Group 1 the index was 2.6 times higher than that of the PHP Group, 2.8 times higher within Group 2, 3.0 times higher within Group 3, in patients within Group 4 - 3.5 times higher. The degree of SPA (Spontaneous Plate Aggregation) in patients with isolated course COLD is probable growth compared with PHP Group - 2,5 times higher within Group 1, in patients with the accompanying CERD within Group 2 - 3.2 times higher, in patients within Group 3-3,6 times higher. CONCLUSION: Exacerbation of COLD is accompanied by activation of iNOS and hyperproduction of NO together with the imbalance of blood aggregation properties.

Key words: COLD, GERD, endothelial dysfunction.

\section{HOW THE LIPID PROFILE IN A GROUP OF HYPERTENSIVE PATIENTS IS} INFLUENCED BY BLOOD PRESSURE

Delia Melania I. Popa', Alexandru-Dan R. C. Costache', Nicoleta M. Dumitrescu', Stefan D. Ciupilan', Ovidiu Mitu', Irina-Iuliana Costache', Antoniu-Octavian Petriș', Prof. Florin Mitu'

"Grigore T. Popa" University of Medicine and Pharmacy, Iași, Romania.

BACKGROUND: Arterial hypertension and dyslipidemia are important risk factors for cardiovascular disease, whose coexistence is often observed in daily clinical practice. The purpose of our study is to evaluate the differences of lipid profile, blood pressure (BP) profile and the influence of risk factors in a group of hypertensive patients. MATERIALS AND METHODS: 140 patients with essential hypertension have been evaluated over a period of two years. Their evaluation included the cardiovascular risk factor profile, with specific accent on the lipid parameters, left ventricular mass index (LVMI) through echocardiography and the values of systolic and diastolic BP (SBP, DBP) both at hospital admission and discharge. The average age was $69.61 \pm 10.57$ years. RESULTS: The admission BP had a better correlation with hypercholesterolemia than the BP at discharge: SBP-total cholesterol $(r=0.16$, $\mathrm{p}=0.186)$, DBP-total cholesterol $(r=0.29, \quad \mathrm{p}=0.014)$, SBP-HDL $\quad(r=-0.31$, $\mathrm{p}=0.011)$, DBP-HDL $(r=-0.40, \quad p=0.001)$. All patients with high grades of arterial hypertension had all the lipid parameters increased, total cholesterol having the best statistical significance $(149.38 \pm 40.04$ - grade 1 vs. $\quad 197.29 \pm 54.75$ - grade $2,187.88 \pm 44.29 \mathrm{mg} / \mathrm{dl}$ - grade $3, \mathrm{p}=0.015$ ). However, LVMI was not significantly different according to BP grade and did not correlate with lipid markers. The hypertensive patients' group with newly diagnosed diabetes had higher LDL levels (121.36 \pm 39.84 Vs. $97.31 \pm$ $37.51 \mathrm{mg} / \mathrm{dl}, \mathrm{p}=0.023)$, while the other risk factors were not apparently associated with significantly different lipid values. CONCLUSION: Our study concluded that high BP values at admission, as well as higher grades of hypertension are associated with increased levels of dyslipidemia and hypertensive patients with high lipid values show an increased risk for diabetes development.

Key words: arterial hypertension, blood pressure, dyslipidemia, cholesterol.

\section{IN VITRO EFFECT OF MAGNESIUM SULFATE ON ISOLATED RAT ILEUM}

Igor Aco Krnjić', Šeherzada (Šemsudin) Halilović, Dragana Drakul², Dragana Sokolović2, Slobodan Milovanović ${ }^{2}$

"Carol Davila" University of Medicine and Pharmacy, Bucharest, Romania. ${ }^{2}$ University of East Sarajevo, Republika Srpska, Bosnia and Herzegovina.

BACKGROUND: Magnesium sulfate (MgSo4) is largely used as osmotic laxative but its direct effect on the ileum has not been investigated so far. It is well known that magnesium ion $\left(\mathrm{Mg}_{++}\right)$is a modulator of $\mathrm{Na}+, \mathrm{K}+$ ion transport systems and blockers of Ca-channels in numerous tissues. Activation of $\mathrm{Na+-K+-ATPase}$ pump with $\mathrm{Mg++}$ ions are essential for regulation of $\mathrm{Na}+$ and $\mathrm{K}+$ transport across cell membranes, including those found in cardiac and vascular smooth muscle cells. $\mathrm{Mg}++$ activates an $\mathrm{Na+}$ $\mathrm{K}+-$ ATPase pump which in turn plays a major role in regulating $\mathrm{Na}+-\mathrm{K}+$ transport in numerous tissues. Magnesium plays an important role in a 
large number of cellular processes by acting as a cofactor in enzymatic reactions and transmembrane ion movements. $\mathrm{Mg++}$ also has a laxative effect (osmotic) by causing swelling of the intestinal contents. The aim of our experiment was to investigate the effect of $\mathrm{MgSO}_{4}$ on ES-induced activity of isolated rat ileum and to check if $\mathrm{KV}$ and BKCa channels take a part in that effect. MATERIALS AND METHODS: We used isolated ileum from male and female rats. The experiments were performed on isolated rat ileum in vitro in the organ bath. $1.5 \mathrm{~cm}$ long segments of ileum were incubated in aerated Tyrod's solution at $37^{\circ} \mathrm{C}$. Ileum was connected to isometric transducer. Electrical stimulation was used to induce activity of the isolated rat ileum. In order to examine participation of $\mathrm{Kv}$ ant $\mathrm{BKCa}$ channels in the mechanism of action of $\mathrm{MgSO}_{4}$ (10-4 -3 $\mathrm{X}_{10-2} \mathrm{M}$ ) we were adding $4 \mathrm{AP}$ (10-3), TEA (10-5 M), 20 minutes before MgSo4. RESULTS: Results showed that $\mathrm{MgSO}_{4}$ on the concentration dependent way inhibits ES induced activity of rat ileum. We found no significant statistical difference if its mechanism of action is influenced by voltage-dependent channels or calcium channels. CONCLUSION: Our results indicate that MgSO4 has direct inhibiting effect on the ES induced activity of rat ileum. Opening of Kv and BKCa channels does not contribute to the effect of $\mathrm{Mg++}$ and it is needed further investigation in order to understand principle and mechanism of its effectiveness.

Key words: magnesium sulfate, relaxation, ileum.

66. IN VITRO EFFECT OF MAGNESIUM SULFATE ON ISOLATED RAT RENAL ARTERY

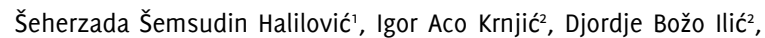
Dragana Drakul3 ${ }^{3}$, Slobodan Milovanovićs ${ }^{3}$ Dragana Sokolović 3

'Faculty of Pharmacy University in Belgrade, Serbia. ${ }^{2 " C a r o l ~ D a v i l a " ~ U n i v e r s i t y ~}$ of Medicine and Pharmacy, Bucharest, Romania. 3University of East Sarajevo, Republika Srpska, Bosnia and Herzegovina.

BACKGROUND: Magnesium ion $\left(\mathrm{Mg}_{++}\right)$is essentially an intracellular cation but is present in serum and interstitial body fluid too. Activation of $\mathrm{Na}+-\mathrm{K}+$ ATPase pump with $\mathrm{Mg++}$ ions are essential for regulation of $\mathrm{Na}+$ and $\mathrm{K}+$ transport across cell membranes, including those found in cardiac and vascular smooth muscle cells. Mg++ activates $\mathrm{Na+-K+-ATPase} \mathrm{pump} \mathrm{which}$ in turn plays a major role in regulating $\mathrm{Na}+-\mathrm{K}+$ transport in numerous tissues. Magnesium plays an important role in a large number of cellular processes by acting as a cofactor in enzymatic reactions and transmembrane ion movements. The aim of our experiment was to investigate the relaxing effect of magnesium on isolated rat renal artery. We tried to check if endothelium and KATP channels take a part in the relaxing effect of magnesium. MATERIALS AND METHODS: We used renal arteries isolated from male and female rats. Each ring was gradually stretched to the optimal tension ( 1.65 grams) and an isometric transducer registered mechanical contractions. Integrity of endothelium was confirmed by acetylcholine. We added $\mathrm{MgS} 04$ in a cumulative manner (10-4 -3X $10-2 \mathrm{M}$ ) on stable tonus produced by phenylephrine (10-5 M). In order to produce optimum inhibition of KATP channels we were adding Clb (10-5 M) 20 minutes before MgSO4. RESULTS: Pretreatment with glibenclamide produced potentiation of relaxing effect of $\mathrm{Mg++}$ on rat renal artery. The effect of magnesium on the isolated rat renal arteries was the same in the presence and absence of the endothelium. Results showed that MgSO4 relaxed the rat renal artery on the concentration dependent way. CONCLUSION: Our results indicate that $\mathrm{MgSO} 4$ has a relaxing effect on isolated rat renal artery. Endothelium delivered factors which don't contribute to relaxing effect on MgSo4 on the renal arteries. Surprisingly, blocking the KATP channels by Glb potentiated the relaxing effect of MgSo4. This effect deserves furthe investigation in order to understand principle and mechanism of it.

Key words: magnesium sulfate, relaxation, renal artery.

\section{IN VITRO RELAXING EFFECT OF SATUREJA MONTANA TINCTURE ON} ISOLATED RAT ILEUM

Andrej Lj. Milaković', Ognjen B. Raljić', Dragana Drakul', Slobodan Milovanović1, Dragana Sokolović

'University of East Sarajevo, Republika Srpska, Bosnia and Herzegovina.

BACKGROUND: Satureja montana from Lamiaceae Family has a long history of use in traditional medicine in the therapy of stomach and intestinal diseases. In this study we examined the in vitro ability of Satureja montana tincture (SMT) to inhibit the electrical stimulation (ES) induced activity of rat ileum. By using different inhibitors, we tried to understand the mechanism of action of SMT on rat ileum. MATERIALS AND METHODS:
Experiment was performed in vitro on isolated rat ileum in an organ bath $(1.5 \mathrm{~cm}$ long segments of ileum were incubated in aerated Tyrod's solution at $37^{\circ} \mathrm{C}$ ). ES was used to induce the rhythmic activity of rat ileum, which was connected to transducer. On the stable activity of isolated ileum, we added an increasing concentrations of SMT. In the series of experiments with antagonists (4-AP, TEA and L-NAME), they were added half an hour before the first concentration of SMT, after which the rising concentrations were given. RESULTS: Our results showed that SMT inhibits, in a concentration-dependent way, the ES induced activity of isolated rat ileum. NO/CGMP system takes a part in the mechanism of action of Satureja Montana on isolated rat ileum because L-name, an inhibitor of NO-synthase, statistically significantly antagonized the effect of SMT. The effect is also related to voltage-dependent potassium channels because 4 -aminopyridine (4-AP), an antagonist of voltage-dependent $K$ channels, reduces the effect of tincture too. Results indicate that Ca-dependent $K$ channels don't participate in the effect of the tincture, because TEA (tetraethylammonium) inhibitor of those channels didn't significantly affect the activity. CONCLUSION: Results indicate that some ingredients of Satureja montana have relaxing effects on isolated ileum and have a potential for treating gastrointestinal spasm.

Key words: Satureja montana, tincture, ileum, organ bath.

\section{IN VIVO ANTI-DIARRHEAL EFFECT OF SATUREJA MONTANA IN RATS}

Ognjen B. Raljić', Djordje Božo llić ${ }^{2}$, Andrej Lj Milaković', Dragana Drakul', Slobodan Milovanović', Dragana Sokolović ${ }^{1}$

'University of East Sarajevo, Republika Srpska, Bosnia and Herzegovina. ${ }^{2}$ Faculty of Pharmacy University in Belgrade, Belgrade, Serbia.

BACKGROUND: Like other species of genus Satureja, Satureja montana shows great potential for having medicinal properties, since it has been widely used to treat diseases and symptoms such as cramps, nausea, diarrhea, as well as bacterial and fungal infections. This study aims to see if Satureja montana essential oil (SMEO) has the potential to reverse castor oil-induced diarrhea in young Wistar rats. MATERIALS AND METHODS: The experiment was performed in vivo on male and female Wistar rats (average weight of $50 \mathrm{~g}$ ), which were separated into two groups (experimental and control). During the night, the experimental group was given SMEO emulsion (1:100 essential oil in water), while the control group was given water. The following morning, both groups were given castor oil $(0,5 \mathrm{ml}$ per os) to induce diarrhea, and during the next three hours, the number of diarrheas was recorded in both groups. RESULTS: Results showed that the experimental group, which had been given SMEO emulsion had statistically significantly fewer diarrheas than the control group. CONCLUSION: Our results indicate that some of the ingredients of SMEO have the ability to antagonize diarrheal effect of castor oil in young Wistar rats and that it deserves further research to understand the principle and mechanism of its effectiveness.

Key words: Satureja montana, essential oil, castor oil, diarrhea.

\section{KIDNEY DISEASE, HEART FAILURE AND SURVIVAL PROGNOSIS - DANGEROUS LIAISONS}

Florescu N-L Daniela-Gabriela', Buzea Adrian MD,PhD ${ }^{1,2}$, Delcea Caterina MD, $\mathrm{PhD}^{1,2}$, Dan Cheorghe Andrei MD, $\mathrm{PhD}^{1,2}$, Daha Ioana $\mathrm{MD}, \mathrm{PhD}^{1,2}$

"Carol Davila" University of Medicine and Pharmacy, Bucharest, Romania. ${ }^{2}$ Colentina Clinical Hospital, Bucharest, Romania.

BACKGROUND: Kidney disease is an independent risk factor for cardiovascular events. The correlation between renal impairment severity and the different groups of heart failure (HF) was, however, insufficiently characterized. MATERIALS AND METHODS: Our aim was to evaluate the impact of different degrees of renal dysfunction on mortality of HF patients, stratified by the three types of HF: with reduced ejection fraction (HFrEF), mid-range EF (HFmrEF) and preserved EF (HFpEF). HF patients admitted to our clinic from January 2011 to December 2014 were included. Acute coronary syndromes, pulmonary embolisms, infections, malignancy, readmissions and in hospital mortality were excluded. Estimated glomerula filtration rate (eGFR) was calculated with the CKD-EPI formula. Survival status was assessed in August 2018, after a mean follow-up period of 5.5 years. RESULTS: Our sample consisted of 1287 patients, $51.6 \%$ female, with 
a mean age of $72.14 \pm 10.49$ years. $30.4 \%$ had $\mathrm{HFrEF}, 29.8 \%$ with $\mathrm{HFmrEF}$ and $39.8 \%$ had HFpEF. $37.11 \%$ had eGFR $<60 \mathrm{mg} / \mathrm{mmol}$. All-cause long-term mortality was $43.8 \%$. eGFR was proportional to the EF: patients with HFrEF had the lowest mean eGFR of $65.10 \pm 22.64 \mathrm{ml} / \mathrm{min}$, followed by HFmrEF with $66.29 \pm 23.90 \mathrm{ml} / \mathrm{min}$ and HFpEF with $72.07 \pm 22.22 \mathrm{ml} / \mathrm{min}$ ( $\mathrm{p}$ for trend $<0.001$ ). Patients with an eGFR $60 \mathrm{ml} / \mathrm{min}$ had an odds ratio of $2.47(95 \% \mathrm{Cl} 1.95-3.13$, $\mathrm{p}<0.001)$ of mortality, while those with an eGFR $<30 \mathrm{ml} / \mathrm{min}$ had an OR of 4.15 ( $95 \% \mathrm{Cl} 2.36-7.29, \mathrm{p}<0.001)$. In HFrEF patients, an eGFR $<60 \mathrm{ml} / \mathrm{min}$ associated a risk of mortality of $1.93(95 \% \mathrm{Cl} 1.28-2.91, \mathrm{p}=0.001)$ and an $\mathrm{eCFR}<30 \mathrm{ml} / \mathrm{min}$ a risk of $3.13(95 \% \mathrm{Cl} 1.23-7.95, \mathrm{p}=0.011)$. In HFmrEF patients, an eGFR $<60 \mathrm{ml} / \mathrm{min}$ associated a risk of $1.99(95 \% \mathrm{Cl} 1.16-3.41, \mathrm{p}=0.011)$ and an eGFR $<30 \mathrm{ml} / \mathrm{min}$ a risk of $4.58(95 \% \mathrm{Cl} 1.20-17.43, \mathrm{p}=0.015)$ of long-term allcause mortality. In HFpEF patients, an eGFR $<60 \mathrm{ml} / \mathrm{min}$ associated a 2.78 $(95 \% \mathrm{Cl} 1.79-4.32, \mathrm{p}<0.001)$ risk of all-cause long-term mortality, and an eGFR $<30 \mathrm{ml} / \mathrm{min}$ a risk of 4.26 (95\% $1.40-12.99, \mathrm{p}=0.005)$. CONCLUSION: Renal impairment with eGFR $<60 \mathrm{ml} / \mathrm{min}$ has a strong effect on HF mortality in all groups of different $\mathrm{EF}$, with the highest risk in HFpEF patients with eGFR<30ml/min.

Key words: heart failure, kidney disease, reduces ejection fraction, midrange ejection fraction, preserved ejection fraction.

NT-PROBNP AND SURVIVAL PROGNOSIS IN HEART FAILURE WITH MIDRANGE EJECTION FRACTION: ARE WOMEN AND MEN THE SAME?

Mara F. Bălan', Anamaria Ș. Draghici', Alexandra Ghita', Pîrciulescu C. Nicoleta', Caterina Delcea ${ }^{1,2}$, Adrian Buzea ${ }^{1,2}$, Cheorghe Andrei Dan $^{1,2}$

"Carol Davila" University of Medicine and Pharmacy, Bucharest, Romania. ${ }^{2}$ Colentina Clinical Hospital-Cardiology Department, Bucharest, Romania.

BACKGROUND: NT-proBNP levels are higher in women than men, more likely so in patients with heart failure (HF) with preserved ejection fraction. Since there is no data about gender differences in natriuretic peptides in patients with HF with mid-range ejection fraction (HFmrEF), our objective was to assess the relationship of NTproBNP with mortality in men and women from this population. MATERIALS AND METHODS: Patients with HFmrEF admitted to our clinic from January 2011 to December 2014 were retrospectively enrolled in this study. Acute coronary syndromes, pulmonary embolisms, malignancy, systemic infections, in-hospital mortality and readmissions were exclusion criteria. All-cause long-term mortality was assessed in August 2018, after a mean follow-up of 5.5 years. RESULTS: Our study included 324 patients with a mean age of $73.45 \pm 10.25$ years. $59.13 \%$ were women. All-cause long-term mortality was $40.5 \% .67 .64 \%$ had acute decompensated HF (ADHF). Female patients had higher median NT-proBNP levels (1571, IQR 801-338 vs $1044 \mathrm{pg} / \mathrm{ml}, \mathrm{IQR} 472.1-1941 \mathrm{pg} / \mathrm{ml}, \mathrm{p}=0.0001$ ). Higher median NT-proBNP levels were recorded in women in both ADHF (1885, IQR 931-3815pg/ml vs 1487.5, IQR 571.2-2670pg/ml, $\mathrm{p}=0.03$ ) and stable HF (1010, IQR $420.1-1838 \mathrm{pg} / \mathrm{ml}$ vs 625.8 , IQR $196.2-1110 \mathrm{pg} / \mathrm{ml}, \mathrm{p}=0.04)$. In women with ADHF, NT-proBNP predicted all-cause long-term mortality with an AUC of $0.693(95 \% \mathrm{Cl} 0.620-0.760, \mathrm{p}<0.001)$ and a cut-off of $>2771 \mathrm{pg} / \mathrm{ml}$, Se $49.35 \%$, Sp $81.37 \%$. In men with ADHF, NT-proBNP predicted all-cause longterm mortality with an AUC of $0.743(95 \% \mathrm{Cl} 0.656-0.819, \mathrm{p}<0.001)$ and a cutoff of $>1023 \mathrm{pg} / \mathrm{ml}$, Se $76.09 \%$, Sp $65.33 \%$. In women with stable HF, NTproBNP levels predicted mortality with an AUC of $0.676(95 \% \mathrm{Cl} 0.505-0.819$, $\mathrm{p}=0.041$ ) and a cut-off of $>757.7 \mathrm{pg} / \mathrm{ml}$, Se $91.67 \%$, Sp $53.85 \%$. In men with stable HF, NT-proBNP levels predicted mortality with an AUC of $0.686(95 \% \mathrm{Cl}$ $0.518-0.825, p=0.037$ ) and a cut-off level of > $587.5 \mathrm{pg} / \mathrm{ml}$, Se $76.92 \%$, Sp $53.85 \%$. CONCLUSION: Women with HFmrEF have higher NT-proBNP levels than men. NT-proBNP is a good predictor of all-cause mortality in HFmrEF in both genders, in both stable HF and ADHF, with higher cut-off levels in female patients.

Key words: NT-proBNP, HFmrEF, heart failure, women, men, gender differences, mortality.

71. PECULIARITIES OF BODY WEIGHT INDEX IN PATIENTS WITH ARTERIAL HYPERTENSION WITH COMORBID NONALCOHOLIC STEATOHEPATITIS

Ivanna Vasylivna Rachynska', Olha Mandryk MD, PhD', Aliona Antoniv MD, PhD' ${ }^{1}$, Olexandr Andrusiak MD, PhD' ${ }^{1}$ Oksana Khukhlina' 'Bukovinian State Medical University, Chernivtsi, Ukraine.

BACKGROUND: In the last decade, there has been a rapid increase in the prevalence of nonalcoholic steatohepatitis (NASH), which as a result leads to cirrhosis and is the most common liver disease, accounting $20-30 \%$ in the general population. MATERIALS AND METHODS: The study involved 50 people aged from 35 to 55 years. 25 patients with normal body weight (BMI to $24.9 \mathrm{~kg} / \mathrm{ml}$ ) were diagnosed with NASH, stage II hypertension while in 25 patients NASH was combined with first degree obesity and stage II essential hypertension. The control group consisted of 20 virtually healthy subjects. The obesity diagnosis was made when BMl reached over $30 \mathrm{~kg} / \mathrm{ml}: \mathrm{BMI}=$ weight $(\mathrm{kg})$ / height $(\mathrm{m})$. RESULTS: The patients of all groups had the average daily SAP which was reliably higher than the normal one $(p<0.05)$, not only in the group with concomitant EH and obesity (2) (by $40.9 \%$ respectively), but also in group 1 (by $36.7 \%$ ) of patients with NASH and EH. The analysis of SAP values in the patients from group 2 at night and in the day time indicates the frequent excess of the standard values of SAP at night (by $52.7 \%$ versus $32.7 \%$ in the day time $(p<0.05)$ ). The variability of SAP was increased in both groups, the maximum values were observed in group 2 ( $34.5 \%$ versus $21.4 \%$ in the 1 st one). The patients with obesity and NASH without the second stage EH also had increased immune response of SAP by 1.8 times $(p<0,05)$ compared to the normal values. CONCLUSION: The course of the second stage EH combined with obesity and nonalcoholic steatohepatitis is characterized by prevailed second stage arterial hypertension $(60,0 \%)$, SAP rises mostly at night (by $52,7 \%$ versus $32,7 \%$ in the day time $(p<0,05))$, the variability of SAP is higher in the day time $(34,5 \%$ $(p<0,05))$ with increasing duration of "load pressure" during the day (by 4.1 times $(p<0.05))$, monotonous type of the curve and inadequate reduction in systolic and diastolic blood pressure during the night period (predominant «non-dipper» type).

Key words: Essential hypertension, nonalcoholic steatohepatitis, SAP.

\section{SEROTONIN FOR DIAGNOSING NEUROENDOCRINE TUMORS}

Ana Gabriela G. I. Prada', Tudor Stroie MD, PhD², Alexandru Lupu', Mircea Diculescu ${ }^{1,2}$

'"Carol Davila" University of Medicine and Pharmacy, Bucharest, Romania. ${ }^{2}$ Fundeni Clinical Institute, Bucharest, Romania.

BACKGROUND: Neuroendocrine tumors (NET) are a new and evolving subject, open to discussion and future research. Represent a heterogeneous group of malignancies located in neck, head, lung and abdomen, with a diverse evolution, histology and nomenclature. Five-year survival rate is much higher if the cancer is found early, before it has spread to lymph nodes or distant parts of the body. Consequently, it is very important to describe specific tumor markers that can aid in timely diagnosing these malignancies. The aim of the study was to assess the capacity of serotonin, a neuroendocrine tumor marker, to identify patients by age group and gender. MATERIALS AND METHODS: We carried out a retrospective study on 80 subjects that were followed up by physicians in Gastroenterology Department of Fundeni Clinical Institute, Bucharest. Data collected from patients' medical files were analyzed based on age group, gender and serotonin serum levels. First patient was admitted to Gastroenterology Department in March 2011, while the last was admitted in April 2019. Inclusion criteria for patients were: patients admitted to the Gastroenterology Department and patients diagnosed with NET. Exclusion criteria were: patients without diagnosis of NET; patients that underwent treatment for less than three months; patients that interrupted treatment; patients that did not come for follow-up. RESULTS: Highest prevalence of increased serotonin serum levels was in pancreatic NET in young $(50 \%)$ and adult $(35 \%)$ patients, but not in elderly $(\mathrm{p}<0.01)$. Older and young patients with hepatic NETs, but not adults, showed significantly higher prevalence of increased serotonin $(p<0.01)$, making it a good marker for these location and age groups. Only older patients with pulmonary NETs had high serotonin. Male patients with pancreatic NETs had higher prevalence $(26 \%)$ of increased serum serotonin levels $(p<0.01)$ signifying a gender specific pattern of this tumor marker for pancreatic location. Only male pulmonary NETs patients showed pathological serotonin serum levels, while female patients showed just normal values, and this could again represent a gender effect. CONCLUSION: There is a gender and age group specificity of serotonin for identifying patients with NETs in various locations and this could help in developing better screening programs.

Key words: NET, serotonin, diagnosis, tumor marker, screening.

73. THE DEGREE OF RESPIRATORY FUNCTION DISORDERS IN PATIENTS WITH COLD DEPENDING ON THE SEVERITY OF GERD

Tayisiya Volodymyrivna Krasnova', Inna Dudka'

'Bukovinian State Medical University, Chernivtsi, Ukraine. 
BACKGROUND: The development of gastroesophageal reflux disease (GERD) in patients with the background chronic obstructive lung disease (COLD) is an urgent problem of medicine. The aim was to study changes in the indices of external respiration and clinical manifestations of GERD in patients with COLD. MATERIALS AND METHODS: 40 patients with COLD of the II stage have been examined ( 10 with isolated course - 1st group, 10 with endoscopically negative (EN) GERD - 2nd group, 10 with endoscopically positive non-erosive (EPN) GERD - 3rd group, 10 with endoscopically positive erosive (EPE) GERD - 4th group. Control groups consisted of 12 apparently healthy persons and 10 patients with isolated GERD course (group 5). RESULTS: In patients with EN GERD, the index of $F E V_{1}$ was $75.2 \%$ and differed from the index in apparently healthy persons for $18.6 \%(p<0.05)$, while in patients with EPN GERD the FEV 1 rate $67.1 \%$ was different from that of the previous one and from that in apparently healthy persons (by $27.4 \%$, p<0.05). In patients with EPE GERD, FEV 1 was lower than the proper values by $54.3 \%$ ( $p<0.05)$, and from the index in apparently healthy persons - by $41.2 \%$ ( $p<0.05)$. Heartburn was observed in $67.7 \%$ of the patients with EN GERD, in $93.3 \%$ of the patients with EPN GERD, in $100 \%$ of the patients in the 4 th group. CONCLUSION: For the patients with COLD, in $73.0 \%$ of the cases the manifestations of GERD have been detected. Clinical peculiarities of GERD in patients with COLD are moderate intensity of clinical manifestations and prevalence of the intensity of extraesophageal respiratory symptoms.

Key words: gastroesophageal reflux disease, chronic obstructive lung disease, FEV1.

74. THE IMPLICATIONS OF TOTAL JOINT ARTHROPLASTY IN RHEUMATHOID ARTHRITIS

Sorana-Alexandra O.R. Boncota', Ana Teodora T.R. Ursu', Madalina Duna MD', Diana Mazilu², Denisa Predeteanu²

" "Carol Davila" University of Medicine and Pharmacy, Bucharest, Romania. "Sf. Maria" Clinical Hospital, Bucharest, Romania.

BACKGROUND: To establish differences between patients with rheumatoid arthritis (RA) who have total joint arthroplasty and those with RA withou total joint arthroplasty. MATERIALS AND METHODS: This is a retrospective horizontal clinical study carried-out from the analysis of the medical files existing in the data-base of the "Sf. Maria" Clinical Hospital, Bucharest Romania. RESULTS: We analyzed a total group of 81 patients with RA admitted to hospital for monitoring the disease under treatment. These patients were divided into 2 groups: one group of 37 patients with RA who underwent total joint arthroplasty and the other group of 44 patients with RA with no joint arthroplasty. The mean age of the total group was 64.88 years and the mean duration of the disease was 19,57 years. The mean value of the disease activity (DAS28) of total group was 4.1 (moderate disease activity). In the group of patients with RA and joint arthroplasty, the mean age was 64,65 years and the mean duration of the disease was 19.15 years. The mean value of disease activity was 3.94 (moderate disease activity). The female/male rate was $32 / 5.25$ patients (68\%) were treated with corticosteroids. 19 patients underwent total hip arthroplasty and 15 patients underwent total knee arthroplasty; 3 patients needed both knee and hip replacement. The time until the first prosthesis was 15.05 years $37.83 \%$ of the patients with RA and joint arthroplasty were treated with biologics. In the group of patients with RA and no joint arthroplasty, the mean age was 65.23 years and the mean duration of the disease was 20 years. The mean value of disease activity was 4.28 (moderate disease activity). The female/male rate was $42 / 2$. 36 patients ( $81.1 \%$ ) were treated with corticosteroids. $39 \%$ of patients with RA and no joint arthroplasty were treated with biologics. CONCLUSION: In contrast to the patients with RA and no joint arthroplasty, the patients with RA and joint arthroplasty received less corticosteroids and biological drugs, which explains that the total joint arthroplasty performed at the optimum moment with the removal of the hypertrophic synovitis decreases the exposure of patients to medications with important adverse effects.

Key words: RA, joint arthroplasty, corticosteroids, biologics.

75. THE INDICATORS OF THE LIVER'S DYNAMIC STATE IN PATIENTS WITH NON-ALCOHOLIC STEATOHEPATITIS DEPENDING ON THE PRESENCE OF COMORBID BRONCHIAL ASTHMA AND OBESITY

Igor Ceorgiiovych Stefaniuk', Kachkovskyi Dmytro Lyubomyrovich, Olha Mandryk MD, PhD

'Bukovinian State Medical University, Chernivtsi, Ukraine.

BACKGROUND: In recent years the incidence of bronchial asthma (BA) and non-alcoholic steatohepatitis (NASH) is growing rapidly. Obesity has become epidemic as well. MATERIALS AND METHODS: 50 people aged from 30 to 50 years (average age - 42) were examined, $40 \%$ - men and $60 \%$ - women. Among them in 30 patients with first-degree obesity (BMI over $30 \mathrm{~kg} / \mathrm{m2}$ ) was diagnosed NASH, and in 20 other patients NASH was combined with first-degree obesity and persistent BA of medium severity. The duration of disease ranged from 2 to 6 years. Control group consisted of 20 almost healthy individuals, according to their age and sex. The examination embodied general clinical blood and urine analysis, rapid response to syphilis, glycemic blood profile, chest X-ray, ECG, biochemical test of liver function and ultrasound of the abdomen. RESULTS: In patients of the second group the decreasing De Ritis ratio was revealed (AST / ALT) to $28.2 \%$ ( $p$ $<0.05)$ and in the first group to $23.4 \%(p<0.05)$ in comparison to the USO. The authentic increase of total bilirubin's content in patients of the second group was established up to 2.2 times ( $p<0.05$ ) in comparison to the firstgroup patients, whose bilirubin's content has increased by 1.5 times. The maximum authentic thymol test indices also related to the second group ( $p$ $<0.05$ ). The increased activity of alkaline phosphatase and $\gamma$-GT was found. It was $35.0 \%$ and $30.4 \%(p<0.05)$ in patients with NASH and BA together with obesity against $20.8 \%$ and $19.2 \%$ ( $p<0.05$ ) in patients of the first group. Bile acids' content in blood has also increased by 2.3 and 1.9 times $(p<0.05)$, indicating the presence of cholestasis, which was also observed in the second-group patients. CONCLUSION: In patients with non-alcoholic steatohepatitis together with first-degree obesity and BA of moderate severity of the persistent flow, the content of markers, which are responsible for cytolysis activity of hepatocytes, cholestasis and mesenchimal inflammation, is increasing. It indicates the powerful impact of asthma on the course of NASH.

Key words: steatohepatitis, bronchial asthma.

\section{THE INFLUENCE OF COMPLEX TREATMENT OF PATIENTS WITH} STEATOHEPATITIS AND OBESITY ON CHANGES OF LIPID SPECTRUM

Yana Ivanivna Toderika', Yana Toderika1, Aliona Antoniv MD, PhD', Olexandr Andrusiak MD, PhD', Olha Mandryk MD, PhD', Oksana Khukhlina

'Bukovinian State Medical University, Chernivtsi, Ukraine.

BACKGROUND: The problem of diagnosis and prognosis of the course of nonalcoholic steatohepatitis (NASH) is one of the important problems of internal medicine, it is of general medical and social importance. MATERIALS AND METHODS: 120 patients with NAFLD and I degree obesity were examined. Two groups of patients were formed. The control group including 60 individuals received Metmorfin - $500 \mathrm{mg}$ twice a day, Essentiale $\mathrm{H}$ ( 1 capsule three times a day) during 90 days. The main group of 60 individuals received Metmorfin - $500 \mathrm{mg}$ twice a day, Steatel (orally $10 \mathrm{ml}$ twice a day) for 90 days. RESULTS: The content of cholesterol on the 3oth day of treatment in the control group was found to be $6,0 \%$ lower $(p>0,05)$, in the main group $19,1 \%$, and on the 90 th day of treatment in the control group $-11,5 \%$ lower $(p<0,05)$, in the main group $-28,5 \%$ lower $(p<0,05)$. The content of cholesterol in the main group became normal on the 3oth day of treatment, although it was lower than those of the control group $(p>0,05)$. On the 3oth day of treatment the content of low density lipoproteins (LDLP) was found to become by 1,6 times lower in the main group and the content of triglycerides - by 1,5 times $(p<0,05)$. In the control group changes of triglycerides in the blood were not reliable $(p>0,05)$. Al in-patients of the main group decreased reliably after treatment, and during examination month later after treatment it reached stable normal values. In the contro group Al decreased under the influence of treatment, although it did not reach the normal values. Against the ground of metabolic syndrome concerning correction of the blood lipid spectrum eliminating hyper- and dyslipidemia and atherogenicity index. CONCLUSION: A comprehensive treatment with administration of the medicines Steatel and Metmorfin is effective in the therapy of non-alcoholic fatty liver diseases comorbidity of obesity steatohepatitis are characterized by high levels of disability and mortality due to the development of atherosclerosis of the coronary and cerebral vessels, which are very often fatal.

Key words: steatohepatitis, Steatel, Metmorfin.

77.

THE INTENSITY OF LIPID PEROXIDATION PROCESSES IN PATIENTS WITH CHRONIC OBSTRUCTIVE PULMONARY DISEASE WITH ASSOCIATED GASTROESOPHAGEAL REFLUX DISEASE

Snizhanalvanivna Hnatkovych', Tetiana Volodymyrivna Dudka 
'Bukovinian State Medical University, Chernivtsi, Ukraine.

BACKGROUND: Under the conditions of comorbidity, due to the direct action of ingredients of gastric juice, and the initiation of esophagobronchial reflex, gastroesophageal reflux disease (CERD) results in the development of bronchospasm and inflammatory process in the bronchi, and drugs used at chronic obstructive pulmonary disease (COPD) induce the progression of CERD. MATERIALS AND METHODS: 45 COPD patients have been examined (15 with endoscopically positive non-erosive (EPN) CERD - 1st group, 15 with endoscopically positive erosive (EPE) GERD - 2nd group and 15 without concomitant GERD - 3rd group). RESULTS: The content of malonic aldehyde (MA) in blood plasma exceeded the control values by $37.9 \%(p<0.05)$ in patients of the 2nd group and by $30.8 \%(p<0.05)$ in the 1 st group compared with apparently healthy persons. The indices of MA content in blood plasma and $\operatorname{Er}(p<0.05)$ were changing less intensively in the 3 rd group of patients, although they exceeded the control data by $23.3 \%$ ( $p<0.05$ ). The content of isolated double bonds (IDB) in the blood reliably increased by 2.2 times in the 2nd group of patients, by 1.8 times in the 1st group, by 1.7 times $(p<0.05)$ in the 3 rd group with the presence of intergroup difference $(p<0.05)$. The maximum increase in the activity of the enzymes glutathione peroxidase (GP) and glutathione transferase (GT) by $32.7 \%$ and $45.8 \%$ respectively $(p<0.05)$ compared to control have been determined in the group of patients with EPE CERD among all the groups of patients with EP GERD. CONCLUSION: The intensity of the LPO processes and the lack of antiradical protection factors in COPD patients increase in direct proportion to the degree of esophagus mucous membrane lesion stipulating severity degree of EP GERD.

Key words: COPD, GERD, lipid peroxidation processes, antioxidant protection.

\section{THE INVOLVMENT OF HUMAN PAPILLOMA VIRUS INFECTION IN} PREMATURE BIRTHS

Irina Mihaela P.C. Matache ${ }^{1}$, Florentina A. Duica ${ }^{2}$, Cretoiu ${ }^{1,2}$

'Carol Davila" University of Medicine and Pharmacy, Bucharest, Romania. 2"Alessandrescu-Rusescu" National Institute of Mother and Child Health, Bucharest, Romania.

BACKGROUND: Human Papilloma Virus (HPV) infection is a viral infection that commonly causes skin or mucous membrane growths (warts). There are more than 200 varieties of HPV. Young women are at high risk of HPV infection and changes in the hormonal balance and immune response during pregnancy make this risk even higher. It is of utmost importance to determine how this may interfere with the pregnancy outcome. Through this study, we aim to offer relevant information for the statistical evaluation of the involvement of HPV infection in premature births. MATERIALS AND METHODS: We collected 112 cervico-vaginal samples from 3 groups of people: first group - pregnant women (7-10 weeks of gestation), second group - mothers who gave birth at term, third group mothers of premature newborns. The cells were then collected in PreservCyt Solution (Hologic Corp) and assayed on a CeneXpert IV device (Cepheid, Sunnyvale, CA USA). This device was used to automate and integrate sample processing, detection of target sequences, cell lysis, purification, nucleic acid amplification. Through this method, we performed a qualitative in vitro test for detection of the E6/E7 region of the viral DNA genome from high risk HPV in patient specimens. RESULTS: The number of HPV positive results in the three groups were: $26 / 77$ (first group), 4/17 (second), 3/14 (third). The prevalence of infection with one strain was $66.6 \%$, with two- $27.7 \%$, with three-6.07\%. The most common strains detected were HPV 16 (9.25\%), HPV 18 (5.55\%), HPV 31 ( $4.62 \%)$. CONCLUSION: HPVs proved to be highly effective pathogens, well-adapted to their ecological niches and capable of avoiding immune responses. During pregnancy, HPV infection has been associated with spontaneous abortions, preterm delivery, premature rupture of the membranes, preeclampsia, dysfunctions in the trophoblast viability and of the endometrial cells' adherence. This study will further focus on clinical trials with the major objective of developing medical applications for the non-invasive detection of biomarkers modified by HPV infection during pregnancy. Thus, we will be able to better prevent complications which may lead to premature births.

Key words: HPV infection, premature births, pregnancy monitorization, prenatal diagnosis.

THE STATE OF RBC CATECHOLAMINE DEPOSITING FUNCTION IN PATIENTS WITH COPD AND GERD
Yuliia Mykolaivna Karliichuk', Yuliia Karliichuk', Inna Dudka MD

'Bukovinian State Medical University, Chernivtsi, Ukraine.

BACKGROUND: According to scientific assumption, gastroesophageal reflux in patients with COPD may arise due to background adrenergic imbalance. MATERIALS AND METHODS: The study involved 60 patients, including: 15 patients with COPD (group 1), 15 patients with COPD and endoscopically negative GERD (group 2), 15 patients with COPD and endoscopically positive non-erosive CERD (group 3), 15 patients with COPD and endoscopically positive erosive GERD (group 4). RESULTS: Patients in group 1 are significantly more likely to have Er, which do not contain granules of CA (category I cells), or contain insignificant number (category II cells) compared with control (1.9 times, p <0.05). In patients of group 2, the number of cells without CA granules exceeds the control index by 2.5 times $(p<0.05)$. The percentage of cells containing more than 10 CA granules in this group of patients is reduced by 1.6 times in comparison with the norm $(p<0.05)$. In patients of group 3 is present an increase in the percentage of cells category I and II by 4.0 times ( $p<0.05$ ). Patients in group 4 have the maximum intensity of change: the number of cells without granules of CA exceeds the normative indicators by 3.9 times. CONCLUSION: Patients with COPD and GERD revealed a decrease in the erythrocyte catecholamine depositing function, which contributes to the unbalance of sympathoadrenal system and is an adverse prognostic criterion for the development of erosive GERD.

Key words: COPD, GERD, catecholamine, adrenergic imbalance.

80.

TIME-LAPS TECHNOLOGIES. NEW OPPORTUNITIES FOR IN VITRO EMBRYOS CULTIVATING

Elizaveta Valentinovna Zakharova', Oksana Shurygina MD'

Samara Medical State University.

BACKGROUND: The objective of this study was to establish the necessity of using video monitoring while cultivating embryos in vitro and to study the possibility of identification of normal and pathological development of embryOS. MATERIALS AND METHODS: The incubator with low concentrations of oxygen ( $5 \%$ ) with video monitoring system Emryovisor (West-Trade, Russia) was used for noninvasively video monitoring of human embryos. Embryo cultivating was made individually in micro holes of WoW dishes (Vitrolife, Sweden) using universal Continuous single Culture (Irvine Scientific, USA). RESULTS: In Research group 456 embryos were analysed by video monitoring (the average age of patients was 32,6 years). Monitoring group was made by 512 embryos (the average age of patients was 32,9 years). The quality assessment was made by standard system of key performance indicator. As a result, the average level of blastocyst development and embryo freezings and disposal coefficient was constantly higher in the group with video monitoring. In Research group the level of blastocyst development was $56,9 \%$ ( $52,3 \%$ in Monitoring group), the freezing was $45,0 \%$ (39,6\% in Monitoring group), disposal coefficient was $50 \%$ ( $35,5 \%$ in Monitoring group). The average amount of transfer was 1,2 in Research group and 1,4 in Monitoring group; the pregnancy rate was $42,6 \%$ in Research group ( $38,5 \%$ in Monitoring group). CONCLUSION: It was determined that Time-laps technologies can lower adverse effects of environment increasing the quality of cultivating and as a result developing embryos of excellent and good quality.

Key words: IVF, in vitro, embryo, video monitoring, time-laps.

\section{A STUDY ON EAR REPLANTATION}

Antonia-Elena C. Clivet', Ionut-Daniel D.0. Iancu', Lavinia I. Cheaba', Mihaela Perțea MD PhD'

'"Grigore T. Popa" University of Medicine and Pharmacy, Iași, Romania.

BACKGROUND: Our ears, besides their role in our acoustic sense, provide symmetry and balance to the human face. A highly misshapen or missing auricle significantly affects not only the well-functioning of hearing, but also the harmony of the physionomy. The patient's psycho-emotional state is greatly affected by the aesthetic prejudice that is the traumatic amputation of the auricle. This is why the surgeon must tackle the challenge of the microsurgical replantation of the auricle with great care and resourcefulness. MATERIALS AND METHODS: The cases we present demonstrate an ensamble of techniques that orchestrate successful auricle replantation procedures. Our three male patients, aged between 45 and 56 , who suffered poly-trauma ulterior to work-related incidents are left with 
avulsion-induced (in 2 of the cases, complete, and one case of partial) amputation of the right ( 2 cases) and left (one case) ear. The microsurgical replantations implied end-to-end arterial anastomosis, the de-epithelization of the posterior face of the auricle and the incision of the cartilage, for the purpose of enlarging the receiving area, and post-operatory leech therapy. The leech therapy alternated the biological leech with the biochemical one (represented by local heparin injection), to provide venous drainage in congested territories. Additionally, exposure to heat and anticoagulants were included in post-operatory care for vasodilatation stimulation and antithrombotic prophylaxis, as well as antibiotic prophylaxis. RESULTS: We solved the issue of venous congestion (a result of the impossibility of venous reconstructive anastomosis) through leech therapy that was applied for 5 days. No case was met with Aeromonashydrophila infection. All three of the cases were finalized into naturally-looking auricles with very little alteration on the patients' appearances. CONCLUSION: Considering the impact of the auricle amputation has on a patient and the balance the ears provide to the face, efforts must be made to reconstruct and recover as much of the original aspect as possible, through both microsurgical and non- microsurgical techniques.

Key words: Reconstructive surgery, ear replantation, leech therapy.

\section{BATTLE OF THE ERAS: CLASSICAL VERSUS MODERN APPROACH IN} COARCTATION OF THE AORTA

Oana-Maria G. Paris' ${ }^{1}$, Andreea V. Paval ${ }^{1}$

"Carol Davila" University of Medicine and Pharmacy, Bucharest, Romania.

BACKGROUND: Coarctation of the aorta ( $C O A$ ) is the sixth most common lesion in congenital heart disease, appearing sporadically, and represents a spectrum of aortic narrowing that varies from a discrete entity to tubular hypoplasia. This study aims to compare surgical and endovascular techniques through feasibility and postoperative outcomes and to outline the optimal management strategy of this disease providing insights to approach this straightforward but challenging condition. MATERIALS AND METHODS: Data of 306 patients aged 1 day- 18 years from a number of retrospective 2011-2018 studies outline that the therapeutic decision for CoA has been influenced by the age of the patient, underlying morphology and the presence of associated cardiac lesions. The inclusion criteria were electrocardiogram, transthoracic echocardiography and magnetic resonance imaging evidence of the site of coarctation and collateral flow. The indication for intervention was the presence of systemic arteria hypertension, with a peak-to-peak coarctation gradient $.20 \mathrm{mg} .109$ patients underwent endovascular repair (stent or balloon angioplasty) and 197 went through surgery. RESULTS: Although interventional therapy reduces undoubtedly invasion and hospitalization time ( 6.5 days versus 25.5 days), it increases the incidence of aneurysm formation ( $5 \%$ vs. $3 \%$ ) and recoarctation ( $47 \%$ vs. $24 \%$ ). The mortality rate was lower in the group of patients who underwent endovascular repair(5.5 vs $11 . \%$ ), but the association procedure-mortality was non-significant due to other lesions. More serious post-operative complications were encountered in the surgery group (spinal cord and phrenic nerve injuries), although there was a higher decrease of Doppler gradient after the surgery (70.2 vs. $34.4 \%$ ). CONCLUSION: An individual patient's clinical presentation, anatomy, size, age and presence of associated cardiac anomalies will most certainly continue to heavily influence treatment approach. Surgery has proven to be an effective treatment for native aortic coarctation, whereas balloon angioplasty (BA) is being used more when dealing with a discrete narrowing, but for a minimal invasive procedure placing a stent after a BA will reduce complications. objective comparisons can be made, therefore, it is difficult to identify an "ideal" surgical technique; however, combining the procedures will hopefully lead to a better outcome and reduce complications in the future.

Key words: Coarctation of aorta, endovascular treatment, surgical intervention, balloon angioplasty.

\section{Chronic Wounds: PRF Treatment vs. Standard Treatment}

Rustin C. Maria Madalina', Raluca G.S. Bocai', Nicoleta N. Asavei', Popescu Valentin', Mastalier Bogdan'

"Carol Davila" University of Medicine and Pharmacy, Bucharest, Romania.

BACKGROUND: Platelet rich fibrin (PRF), a second generation platelet concentrate, is known as a healing biomaterial that found its application in lots of surgical fields. Being obtained without any addition, PRF can be considered to generate an accelerate and physiological healing by its prolonged and intensive release of grow factors. Chronic wounds, with a prevalence of $1-2 \%$ in general population, are major health problems impairing the quality of life, causing pain and infectious complications, increasing hospital stay and costs. The use of PRF was recently studied for its benefic properties in regenerative medicine, but many other studies are needed. We aimed to compare the standard treatment for diabetic ulcers and pressure ulcers with the PRF therapy in order to emphasize the safety and effectiveness of this new possibility of treatment. MATERIALS AND METHODS: Our study enrolled 20 patients with chronic wounds between 2017 and 2019, treated at Colentina Hospital. Our cohort was randomly divided into a PRF treated group (2 diabetic ulcers, 6 pressure ulcers) and a standard treated group (12 patients). Patients were followed up weekly for 21 days. Degree of filling and level of shrinkage were assessed. Clinical evaluation on the colorimetric scale was included and the costs for each type of therapy per patient were analyzed. RESULTS: All PRF treated wounds showed significant improvements. The (pink color) colorimetric evaluation revealed that PRF accelerated healing. The average time until wound cover was stable (70-80\%) was 20 days in the PRF treated group. There were reported 3 infectious complications in standard treatment patients and none in the PRF group. The mean hospitalization period for the control group was twice as much as for PRF treated group. Moreover, PRF treatment is 4.36 times cheaper than standard treatment. CONCLUSION: PRF promotes healing and wound closure in all patients. It can be reapplied for complete filling and has a significant antimicrobial effect. It showed excellent results compared to standard protocol treatment in both diabetic and pressure ulcers. The PRF therapy is safe, effective, cheap and easy to achieve. As a result, it can be taken into consideration as baseline treatment in these types of chronic wounds.

Key words: PRF, wound healing, diabetic ulcer, pressure ulcer.

\section{DETERMINATION OF THE ETIOLOGICAL FACTORS IN THROMBOANGIITIS} OBLITERANS, FORMATION OF THE OPTIMAL DIAGNOSTIC AND TREATMENT PLAN AND MONITORING OF PATIENTS WITH THIS DISEASE Artur Ion Munteanu', Ecaterina Igor Caliga', Alexandru Iliadi

I"NicolaeTestemițanu" State University of Medicine and Pharmacy, Chișinau, Moldova.

BACKGROUND: Thromboangiitis obliterans remains a disease with a poorly understood etiology but is associated with smoking, for this we have made a statistic of smokers and non-smokers and the best diagnostic and treatment methods according to the cases presented. MATERIALS AND METHODS: The study is based in the Clinic №1 of the Department Surgery 2 of the State University of Medicine and Pharmacy "Nicolae Testemițanu". A retrospective study was conducted using a statistical method, which is based on the analysis of a data complex, taken from case histories. The study involved 25 patients aged 22 to 65 years. RESULTS: When distributing patients according to symptoms, it was found that patients suffer from: pain - 21 patients $(84 \%)$, intermittent claudication - 10 patients $(40 \%)$ paresthesia - 8 patients $(32 \%)$, ischemic ulcers - 7 patients $(28 \%)$, a cooling feeling in the limbs - 5 patients $(20 \%)$, hypothermia in the extremities - 5 patients $(20 \%)$. Since the disease is related to smoking, a distribution was made patients and on this basis: 22 patients ( $88 \%$ ) are smokers, 3 patients $(12 \%)$ do not smoke. Diagnostic methods that have been used to diagnose occlusion: in 17 patients $(68 \%)$ - angiography with contrast, in 6 patients (24\%) - Doppler ultrasound, in 2 patients (8\%) - CT angiography. The treatment was different depending on the case: 4 patients $(12 \%)$ amputation, 9 patients $(36 \%)$ lumbar sympathectomy, 9 patients $(36 \%)$. lumbar sympathectomy and amputation, 1 patient (4\%) PA revision, 1 patient $(4 \%)$ supra and subpopliteal by-pass, 2 patients $(8 \%)$ conservative treatment. CONCLUSION: Clinical manifestations and angiography are the basis of diagnosis. Quitting smoking is the only way to stop the progress of the disease. Conservative treatment is only symptomatic. Surgical treatment: lumbar and thoracic sympathectomy, revascularization, venous arterialization, omentopexy, stem cells increase peripheral blood flow and reduce the risk of amputations.

Key words: Thromboangiitis obliterans.

85

HIRUDOTHERAPY (MEDICAL LEECH THERAPY) AND ITS EFFICIENCY IN REPLANTATIONS

Adina Ceorgiana A. Para', Remus Andrei C. Tofan', Andreea Maria F.M. Roata', Mihaela Perțea ${ }^{2}$ 
'"Grigore T. Popa" University of Medicine and Pharmacy, Iași, Romania. ${ }^{2}$ Clinic of Plastic Surgery and Reconstructive Microsurgery, "St. Spiridon" Emergency County Hospital, Iași, Romania.

BACKGROUND: The use of the medicinal leech, Hirudomedicinalis, is a theme of ancient and modern medicine. Hirudotherapy provides an alternative route for the removal of pooled venous blood, its main advantages being the reduction of capillary and hydrostatic pressure and the improvement of the reperfusion, while the body works to restore native channels of venous egress. Its efficacy in the treatment of venous congestion ranges between 65-85\%. MATERIALS AND METHODS: Our study includes 23 patients ( 18 men and 5 women) who suffered amputations of fingers and thumb in 19 cases and, in the other 4 , the patients suffered complete amputation of the ear auricle. In all ear replantations, arterioraphy was performed and, just in one case, the venous reconstruction was possible. Even though in 12 cases of digital replantation it was managed to perform the venous reconstruction, the leech therapy was necessary and beneficial. Leech therapy began with the first sign of venous congestion. The leeches were applied at the level of the replanted segment where was created a tegumentary flare for bleeding. Their time of action lasted until their spontaneous detachment. Signs of venous congestion improved after the first day of using leeches. RESULTS: The therapy was maintained on average 5 days. There was no record of Aeromonashydrophila (a gram negative germ located in the gut of the leech) infection and no prolonged bleeding. All the replantation results were good with the survival of the amputated segment. CONCLUSION: Hirudotherapy plays an essential role in postoperative management of the microsurgical replantations for the management of the venous congestion. It is cheap, safe, easy to use and a beneficial therapy.

Key words: hirudotherapy, microsurgical, replantations, arterioraphy, amputations, venous congestion.

86. MINIMALLY INVASIVE SURGERY APPROACH ON CASE OF SOLITARY AORTIC DEFECT

Stefan Mihai Crețu', Andrei Ureche MD, $\mathrm{PhD}^{2}$, Serghei Voitov MD, $\mathrm{PhD}^{2}$, Andrian Rotaru ${ }^{1,2}$, Prof. Aureliu Batrinac ${ }^{1,2}$

'NicolaeTestemițanu" State University of Medicine and Pharmacy, Chișinau, Moldova. ${ }^{2}$ Medpark International Hospital Chișinau, Moldova.

BACKGROUND: Mini-sternotomy for plastic surgery or isolated aortic valve replacement requires rapid recovery with diminished convalescence time, improved cosmetic outcome and lower hospital costs. The basic clinical benefit of a mini-sternotomy implies that the lower half of the ribcage remains intact. The basic conduct of virtually all other aspects of the aortic valve replacement procedure remains the same. Therefore, similar longterm results are expected. MATERIALS AND METHODS: In the period April 2014 - April 2019 in Medpark Hospital were operated 76 patients with severe aortic valve solitary. All patients underwent inverted J-sternotomy, which extended over the sternum handle to the third right intercostal space, without opening the pleural cavity. The ages of the patients ranged from 23 to 77 years, 50 -men and 26 -women. The patients were kept in the Trendelenburg position, used being the bilateral venous cannulation, the common aortic cannulation. All patients benefited from bioprostheses and mechanical prostheses with diameters between 21 and $29 \mathrm{~mm}$. Three patients underwent conversion to conventional sternotomy. RESULTS: The length of stay in intensive care was significantly shorter with 0.61 days in favor of the mini-sternotomy group. There was no benefit in terms of ventilation duration. There was evidence suggesting a reduction in blood loss and length of stay in the hospital in the mini-sternotomy group. This did not prove statistically significant (the reduction with, on average, by $114.4 \mathrm{ml}$ and 2.03 days less hospitalization). Deep sternal infections were not reported. CONCLUSION: Mini-sternotomy for isolated aortic valve replacement significantly reduces the length of stay in cardiac intensive care. Other short-term benefits may include lowering blood loss. At the same time, deep sternal infections were not reported, which is a remarkable result. This approach provides greater comfort to patients in the early postoperative period, with a painful syndrome decreased and a greater desire for early discharge from hospital and all its inherent advantages.
Key words: Mini-sternotomy, Aortic valve replacement, Trendelenburg position, Bioprostheses prostheses, Mechanical prostheses, J-sternotomy Cardiac Intensive Care.

\section{NEGATIVE PRESSURE THERAPY IN SOFT TISSUE LESSIONS FOLLOWED BY} USE OF HYALURONIC ACID PLUS SILVER SULFADIAZINE

Remus Andrei C. Tofan', Andreea Maria F.M. Roata', Adina Georgiana A. Para', Mihaela Perțea ${ }^{2}$

"'Grigore T. Popa" University of Medicine and Pharmacy, Iași, Romania. ${ }^{2}$ Clinic of Plastic Surgery and Reconstructive Microsurgery, "St. Spiridon" Emergency County Hospital, Iași, Romania.

BACKGROUND: This presentation aims to evaluate the results obtained by using negative pressure treatment, followed by silver sulfadiazine and hyaluronic acid applications in cases of soft tissue lesions of various etiologies. MATERIALS AND METHODS: Our study is based on 42 patients with wounds: 19 cases with infected soft tissue defects in the lower leg (7 patients with trophic ulcers and 12 cases of posttraumatic lesions), 21 patients with lesions in the hand, wrist and forearm (12 cases of posttraumatic lesions, 3 of complicated thrombophlebitis due to peripheral catheter and 6 cases with different etiologies). The other 2 cases were wrist septic arthritis with fulminant evolution. In all of the cases, negative pressure therapy (VAC) was applied. After the control and treatment of the infection and after we obtained a granulation wound bed, split-thickness skin graft (15 cases) or secondary epithelialization using hyaluronic acid in combination with silver sulfadiazine was performed. RESULTS: In all 42 cases, the negative pressure treatment, allowed a significant reduction of the healing time with the formation of a good quality granulation wound bed. Also, it decreased the patient pain by reduction of the number of the necessary dressings and avoiding the risks of repeated anesthesia. CONCLUSION: Negative pressure treatment is a first intention solution in skin wounds of different etiologies, infected or not, followed by topical applications with silver sulfadiazine and hyaluronic acid for secondary epithelialization.

Key words: negative pressure therapy, septic arthritis, soft tissue lesion,s hyaluronic acid, silver sulfadiazine.

This work is licensed under a Creative Commons Attribution 4.0 International License

ISSN 2076-6327

This journal is published by the University Library System, University of Pittsburgh as part of the Digital Publishing Program and is co-sponsored by the University of Pittsburgh Press. 